\title{
Proanthocyanidin Dimers and Trimers from Vitis vinifera Provide Diverse Structural Motifs for the Evaluation of Dentin Biomodification
}

Rasika S. Phansalkar, ${ }^{\dagger}$ Joo-Won Nam,${ }^{\dagger, \S}$ Ariene A. Leme,${ }^{\ddagger}$ Li-She Gan,${ }^{\perp}$ Bin Zhou,${ }^{\dagger}$ James B. McAlpine, ${ }^{\dagger}$ Shao-Nong Chen,$^{\dagger}$ Ana K. Bedran-Russo, ${ }^{\ddagger}$ and Guido F. Pauli ${ }^{\dagger} * *$

'Department of Medicinal Chemistry and Pharmacognosy, and Program for Collaborative Research in the Pharmaceutical Sciences (PCRPS), University of Illinois at Chicago, College of Pharmacy, Chicago, Illinois 60612, United States

Department of Restorative Dentistry, College of Dentistry, University of Illinois at Chicago, Chicago, Illinois 60612, United States

${ }^{\S}$ College of Pharmacy, Yeungnam University, Gyeongsan, Gyeongbuk 712-749, Korea

${ }^{\perp}$ College of Pharmaceutical Sciences, Zhejiang University, Hangzhou, Zhejiang, 31005, China

\section{SUPPORTING INFORMATION}


S1: ${ }^{1} \mathrm{H},{ }^{13} \mathrm{C}, \mathrm{COSY}, \mathrm{HMBC}$, and ROESY spectra of 2

S2: ${ }^{1} \mathrm{H},{ }^{13} \mathrm{C}, \mathrm{COSY}, \mathrm{HMBC}, \mathrm{HSQC}$ and ROESY spectra of $\mathbf{3}$

S3: ${ }^{1} \mathrm{H},{ }^{13} \mathrm{C}, \mathrm{COSY}, \mathrm{HMBC}, \mathrm{HSQC}$ and ROESY spectra of 5

S4: ${ }^{1} \mathrm{H},{ }^{13} \mathrm{C}, \mathrm{COSY}, \mathrm{HMBC}, \mathrm{HSQC}$ and ROESY spectra of $\mathbf{6}$

S5: ${ }^{1} \mathrm{H},{ }^{13} \mathrm{C}, \mathrm{COSY}, \mathrm{HMBC}, \mathrm{HSQC}$ and ROESY spectra of 9

S6: HiFSA parameter sets for compounds 1-9

S7: ${ }^{1} \mathrm{H}$ and ${ }^{13} \mathrm{C}$ NMR chemical shifts for dimers $\mathbf{1 ,} 4$ and $\mathbf{7}$ and trimer 8

S8: Chiral chromatographic analysis after phloroglucinolysis

S9: DFT calculated ${ }^{13} \mathrm{C}$ NMR chemical shifts and DP4+ analysis of compound $\mathbf{2}$ and $\mathbf{5}$

S10: Dentin stiffness assay results

S11: Collagenase assay results

S12: Purity analysis of compounds 1-9 using $100 \%$ qHNMR method 


\section{S1}

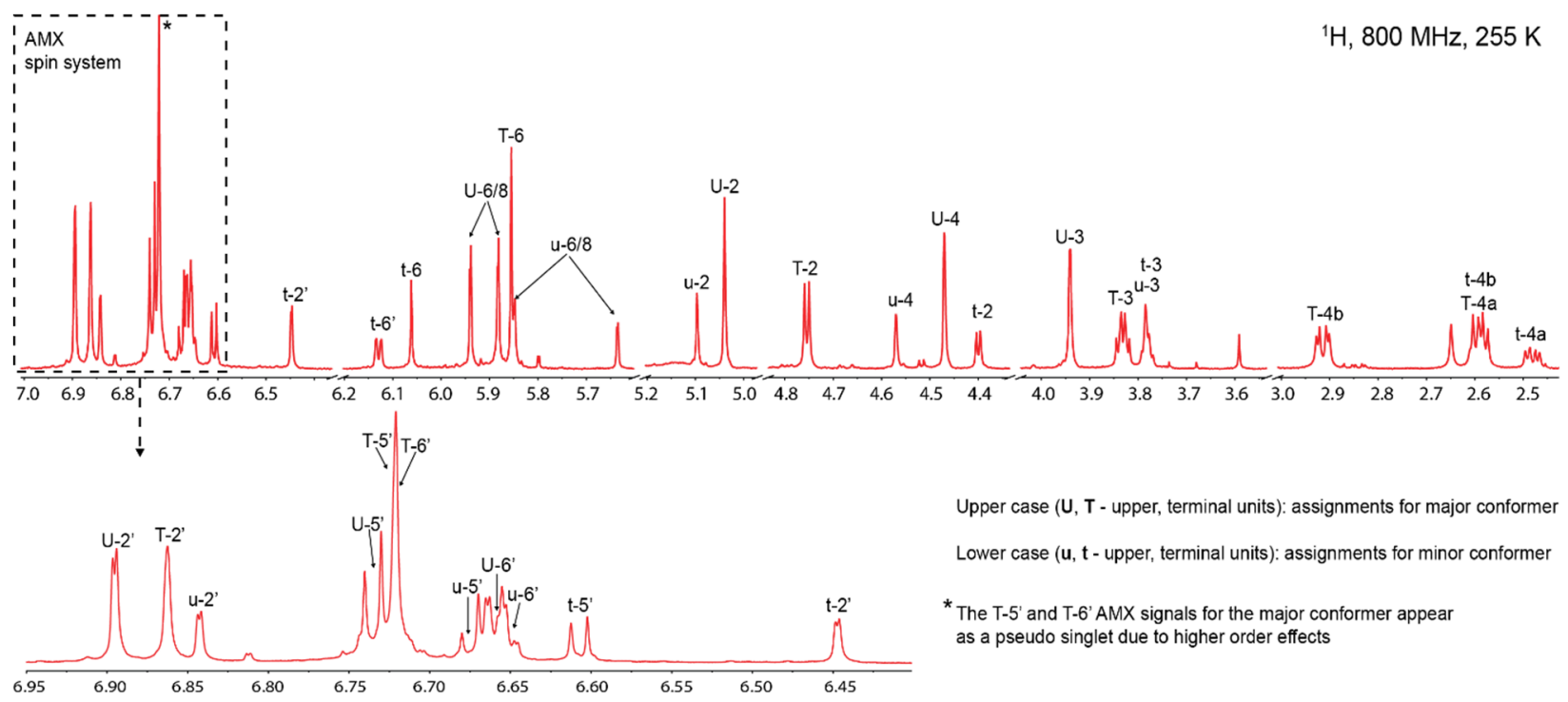

Figure S1 A: ${ }^{1} \mathrm{H}$ NMR signal assignments for compound $\mathbf{2}$ in methanol- $d_{4}$

${ }^{13} \mathrm{C}, 225 \mathrm{MHz}, 255 \mathrm{~K}$

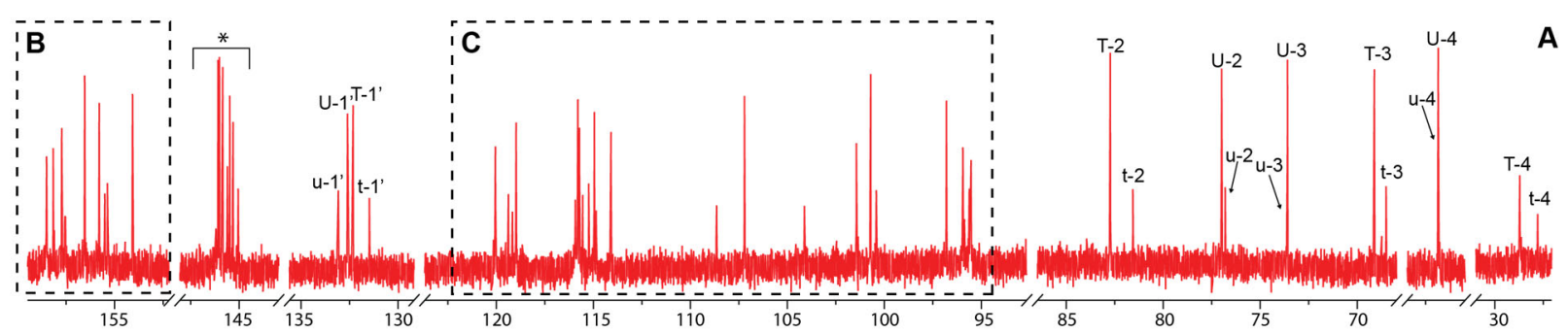

B

C

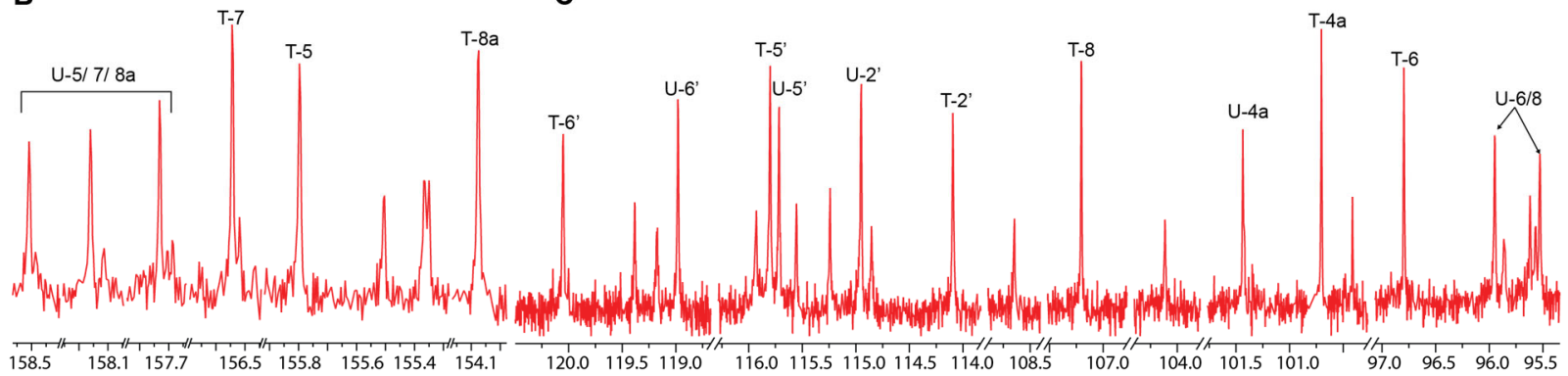

Upper case (U, T - upper, terminal units): assignments for major conformer $\quad *$ Not assigned

Lower case (u, t - upper, terminal units): assignments for minor conformer $\quad \square$ Assignment interchangeable

Figure S1 B: ${ }^{13} \mathrm{C}$ NMR signal assignments for compound 2 in methanol- $d 4$. Panel A: C-ring aliphatic carbons; panel B: A-ring quaternary carbons; panel C: carbons belonging to the B-ring AMX and A-ring AX spin systems 


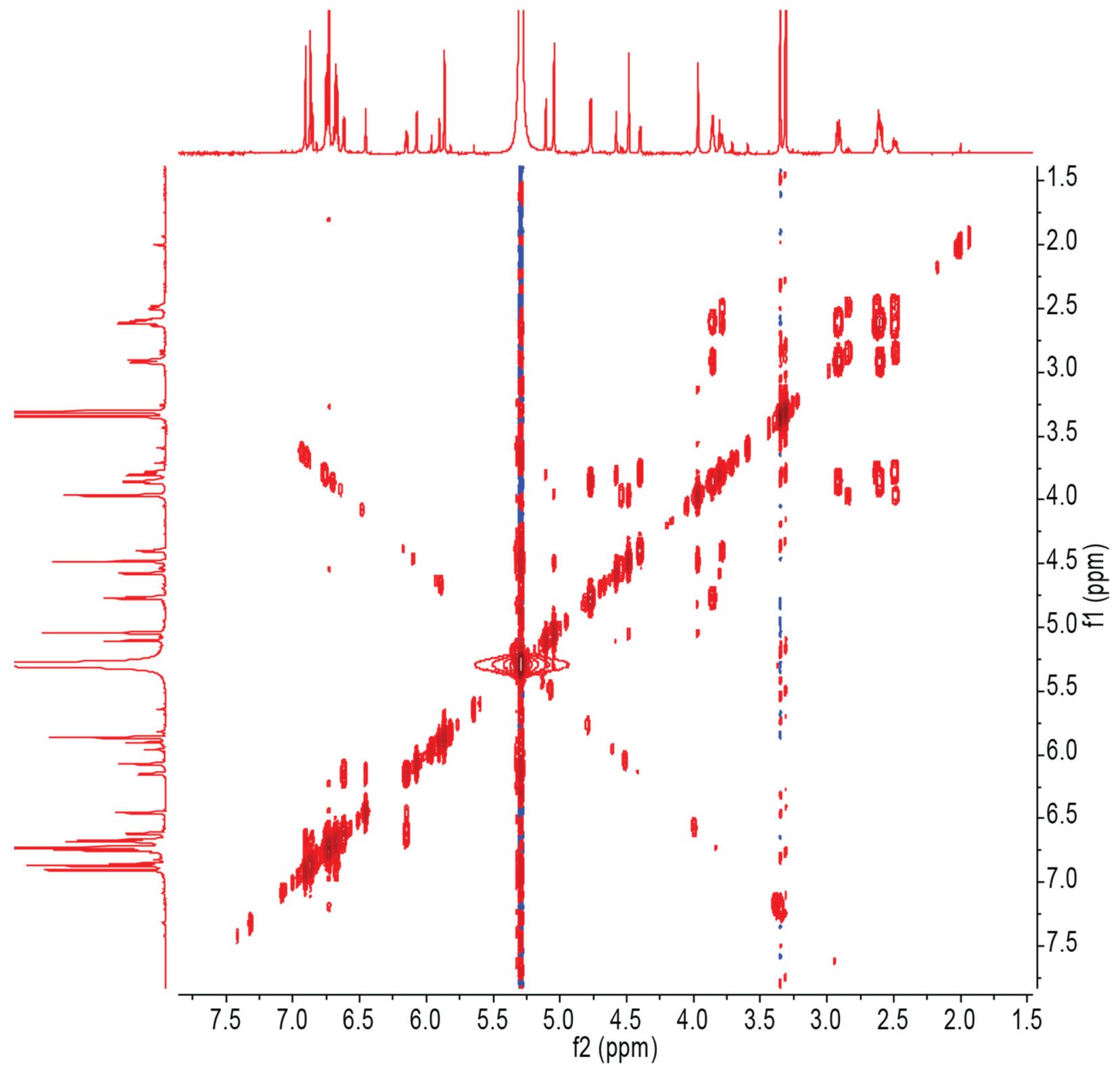

Figure S1 C: Compound 2, COSY spectrum 


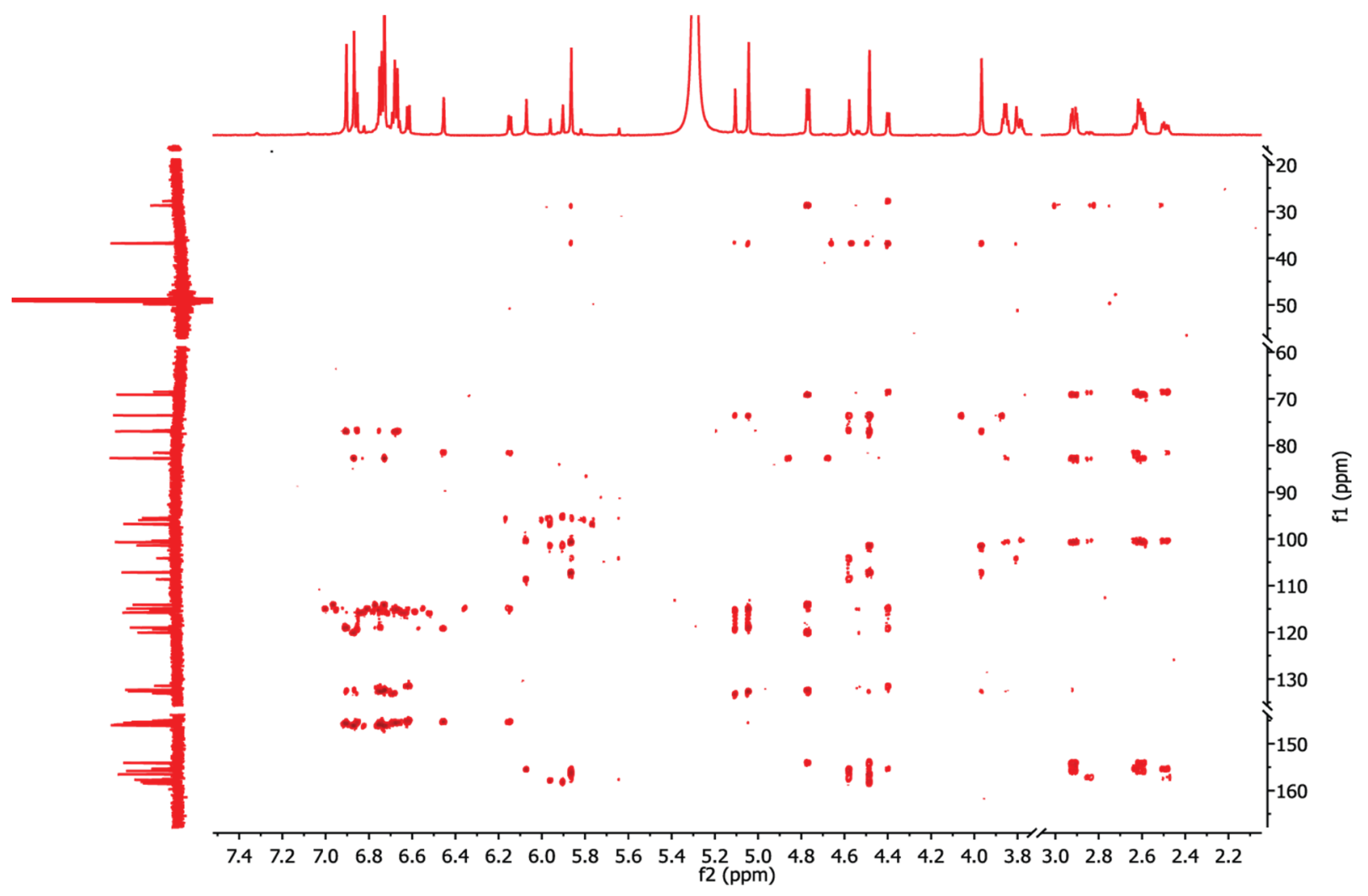

Figure S1 D: Compound 2, HMBC spectrum 


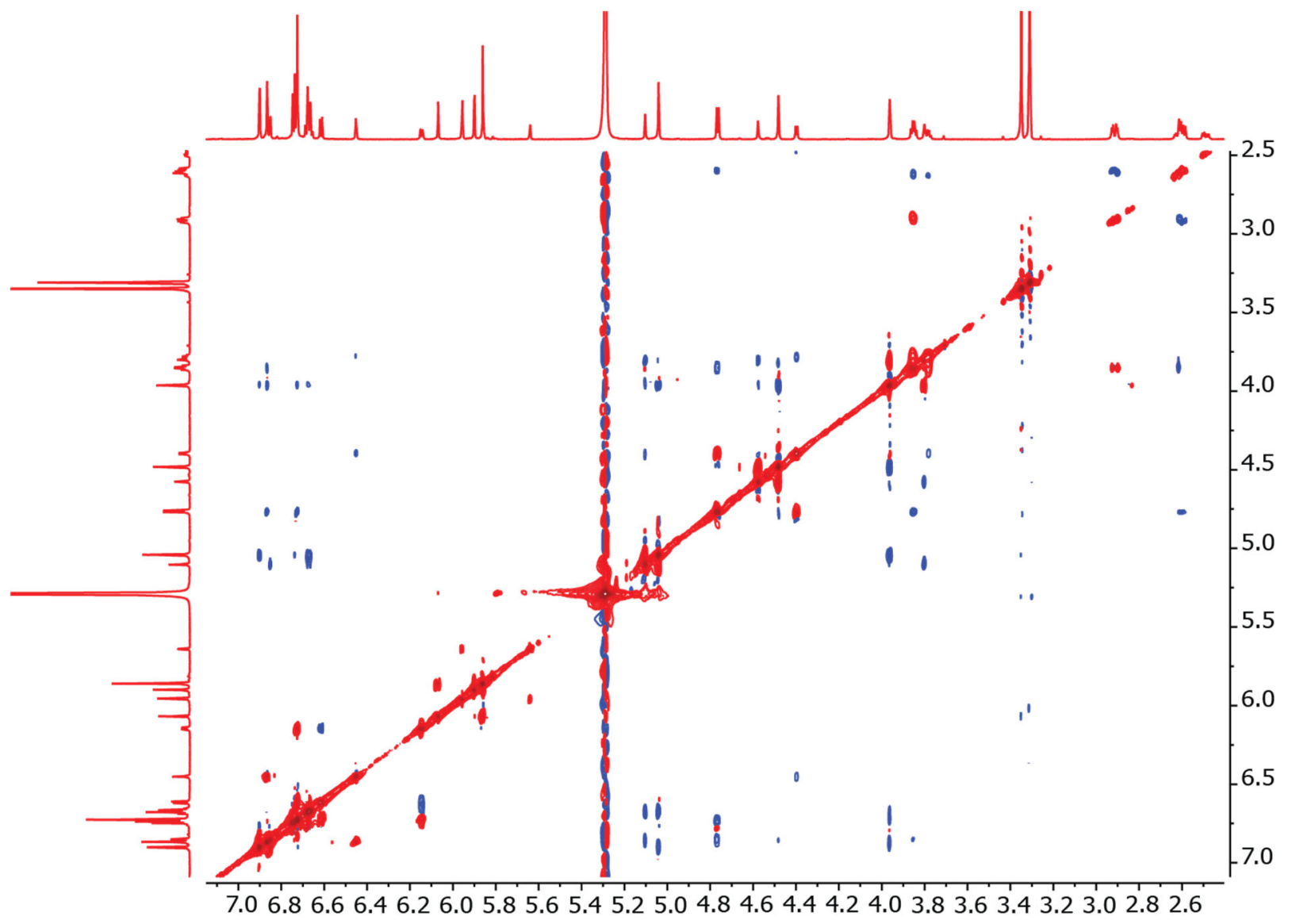

Figure S1 E: Compound 2, ROESY spectrum 


\section{S2}

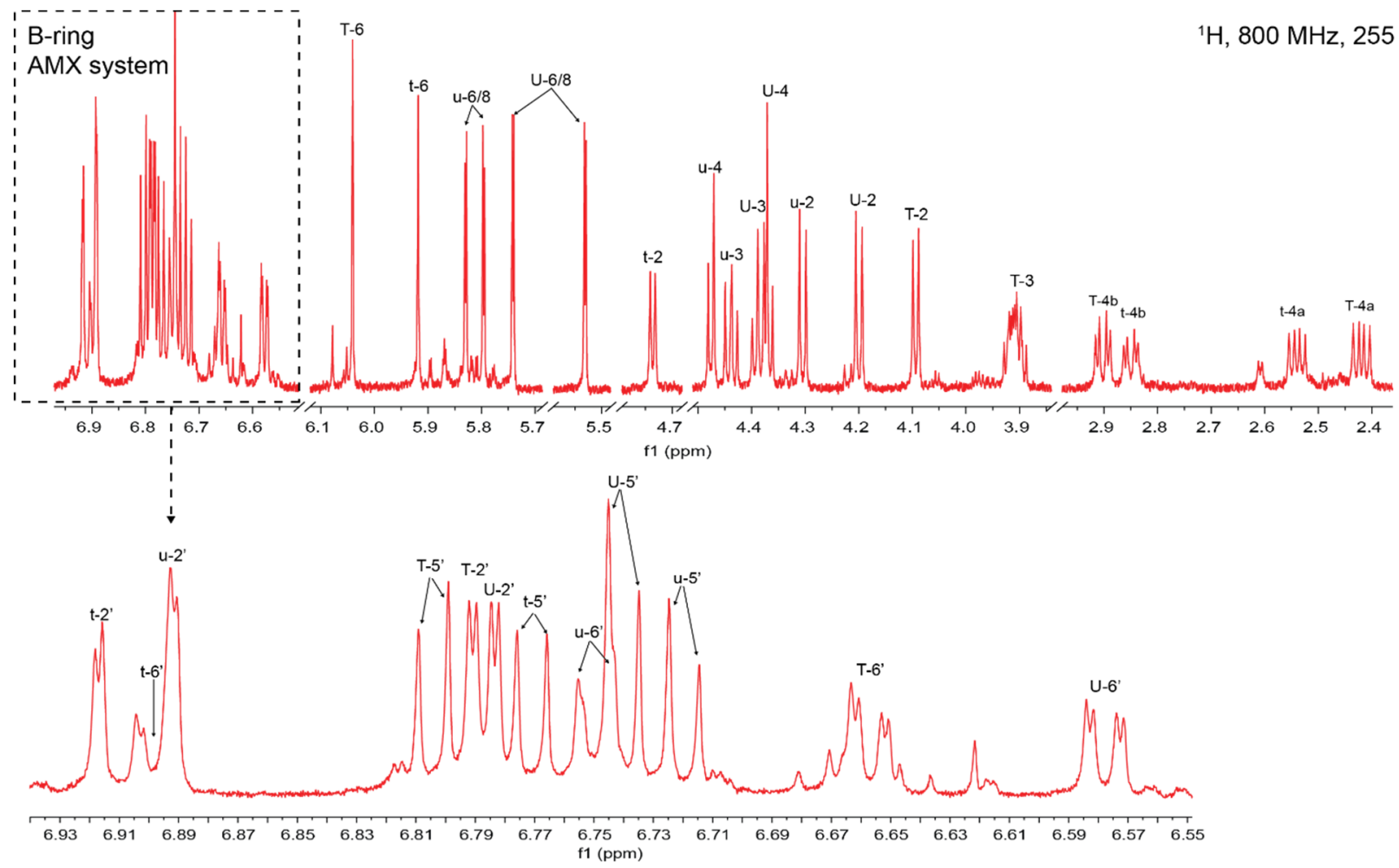

Figure S2 A: ${ }^{1} \mathrm{H}$ NMR spectrum of compound 3 in methanol-d4. The dotted lines: B-ring AMX spin system region. $\mathrm{U} / \mathrm{T}$ and $\mathrm{u} / \mathrm{t}$ : upper and terminal unit of major and minor rotamers.
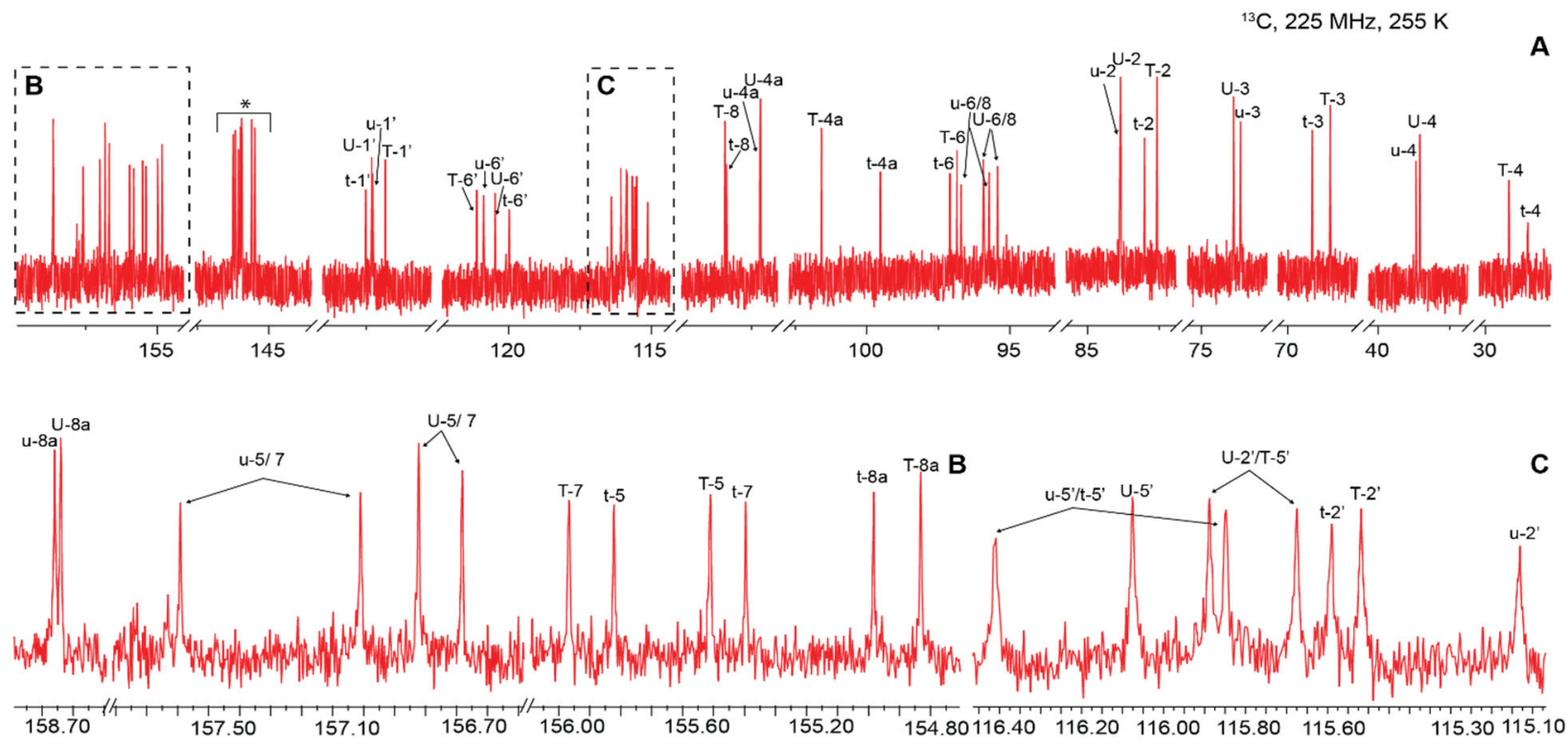

* Not assigned

Figure S2 B: ${ }^{13} \mathrm{C}$ NMR spectrum of compound $3(255 \mathrm{~K})$ in methanol- $d 4$. Panel A: Full ${ }^{13} \mathrm{C}$ NMR spectrum of 3; panel B: A-ring quaternary carbos; panel C: expansion of the B-ring AMX spin system carbons; $\mathrm{U} / \mathrm{T}$ and $\mathrm{u} / \mathrm{t}$ : upper and terminal unit of major and minor rotamers. 


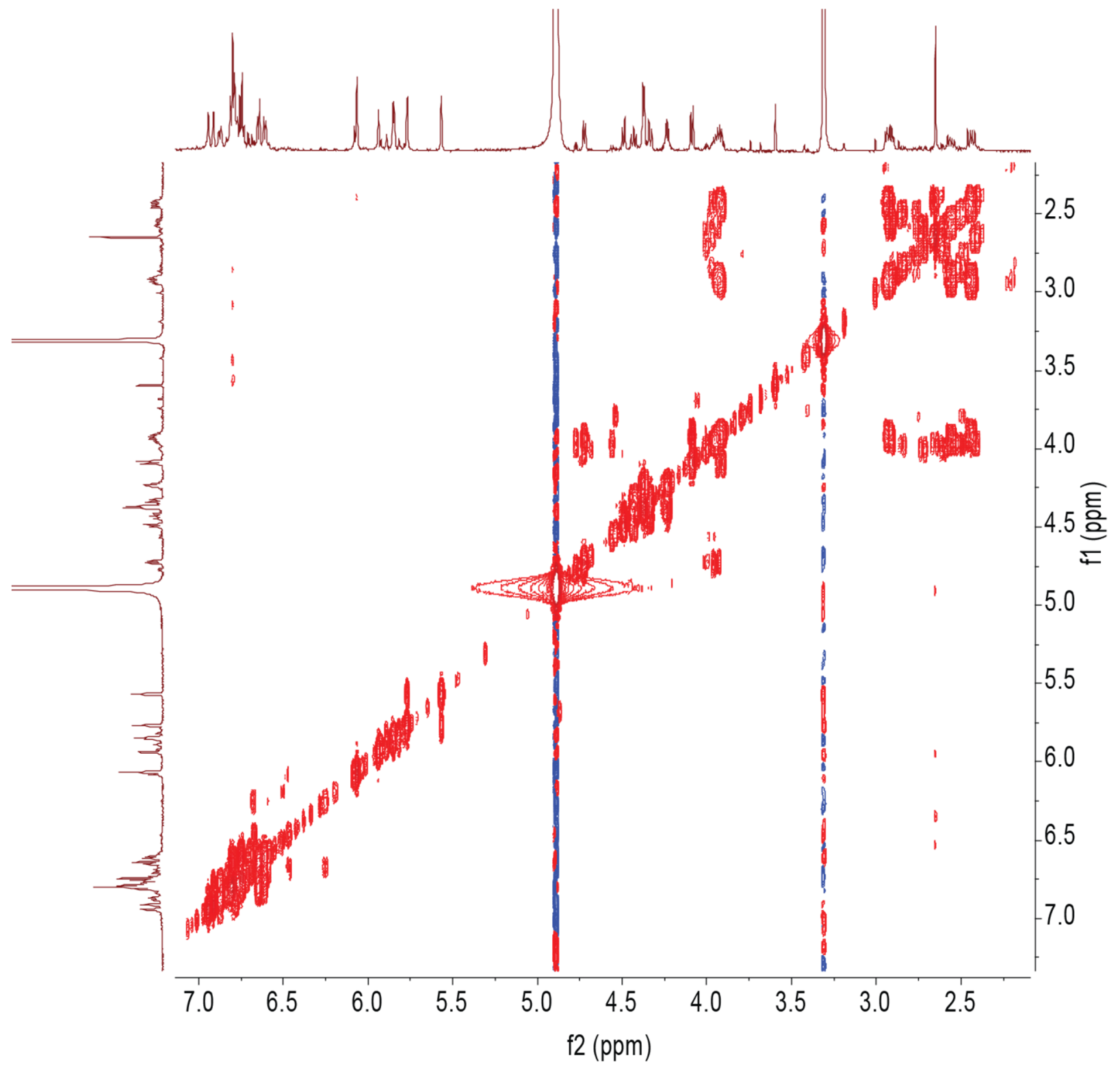

Figure S2 C: Compound 3, COSY spectrum 


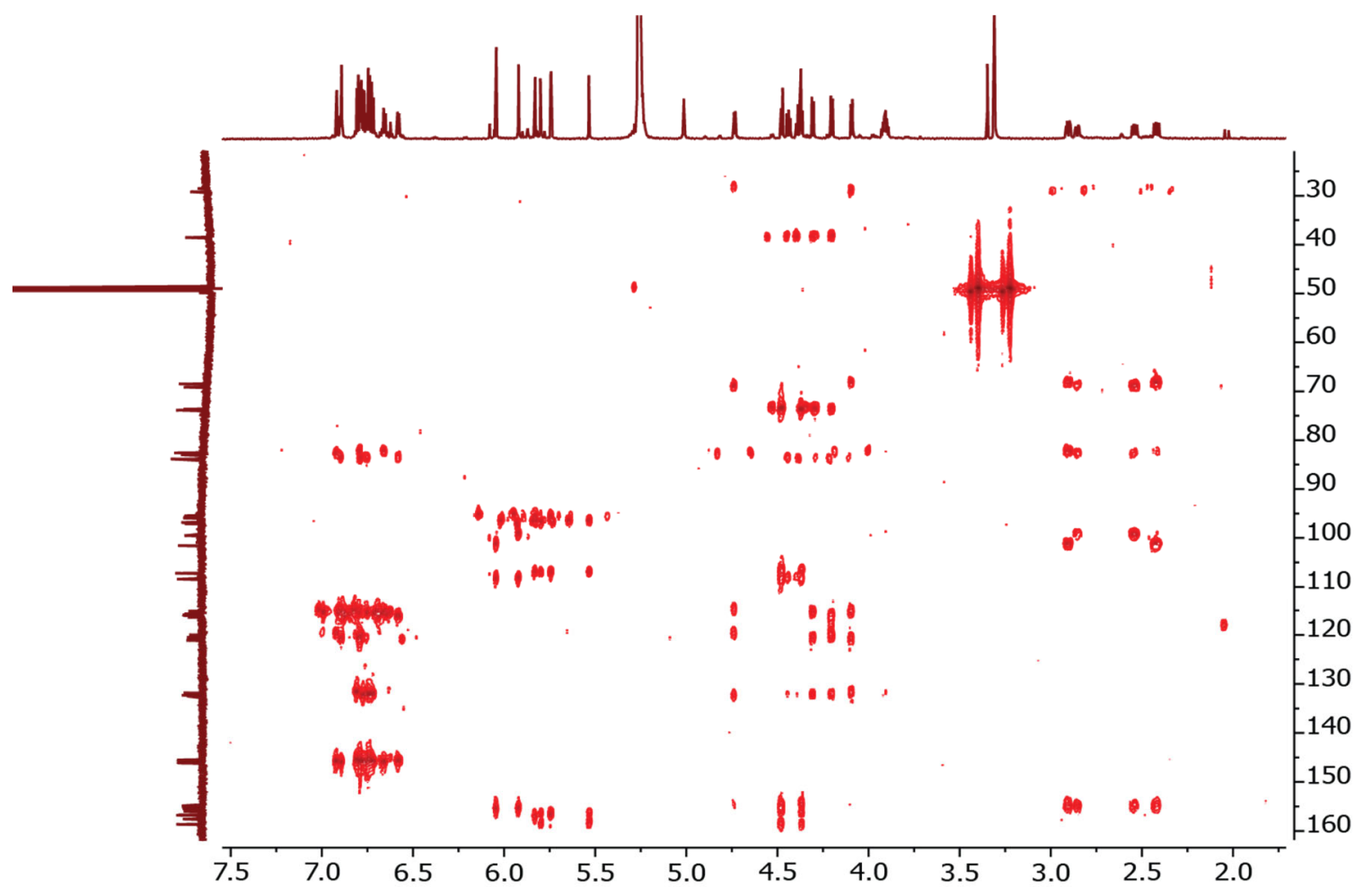

Figure S2 D: Compound 3, HMBC spectrum

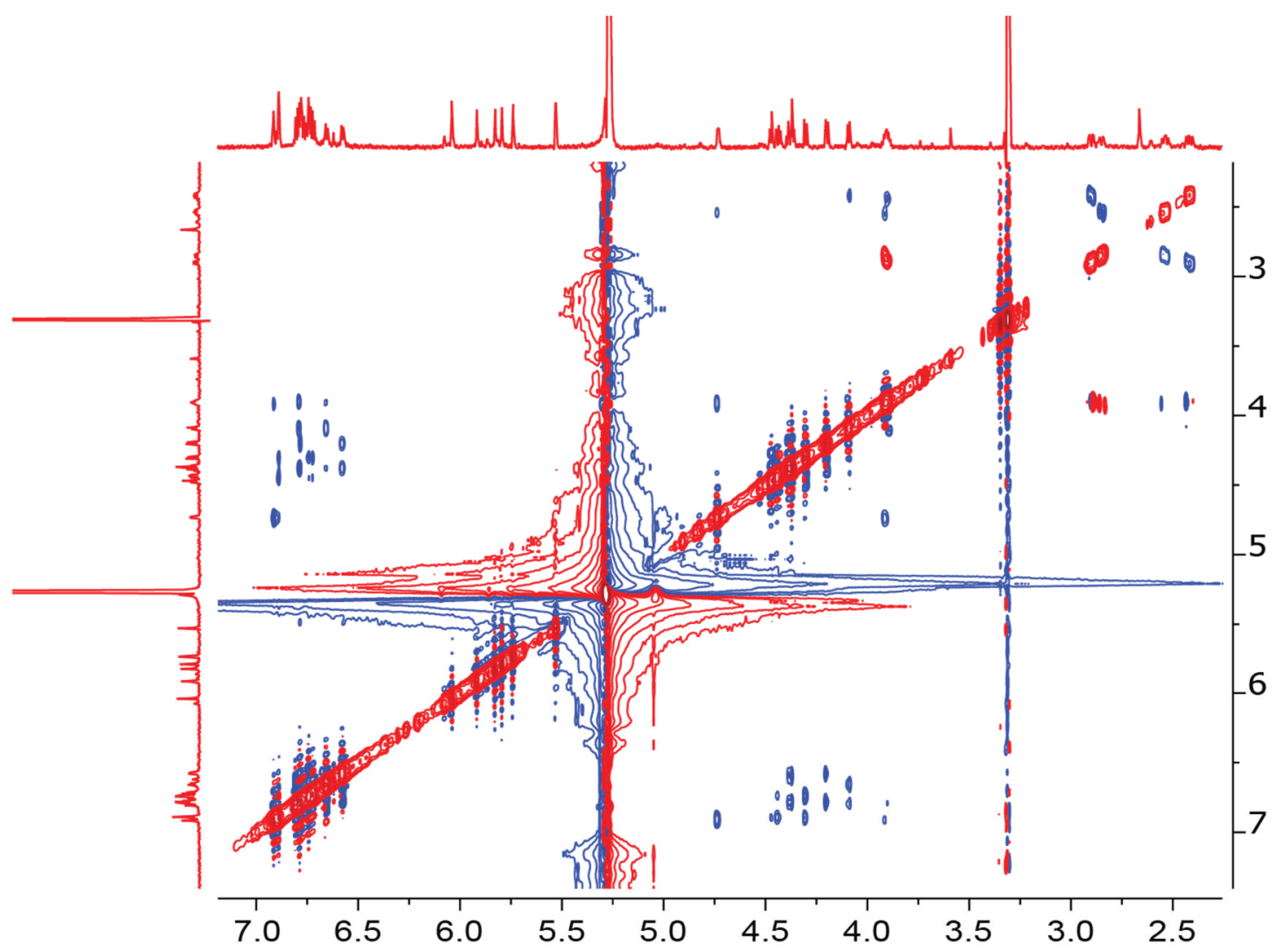

Figure S2 E: Compound 3, ROESY spectrum 

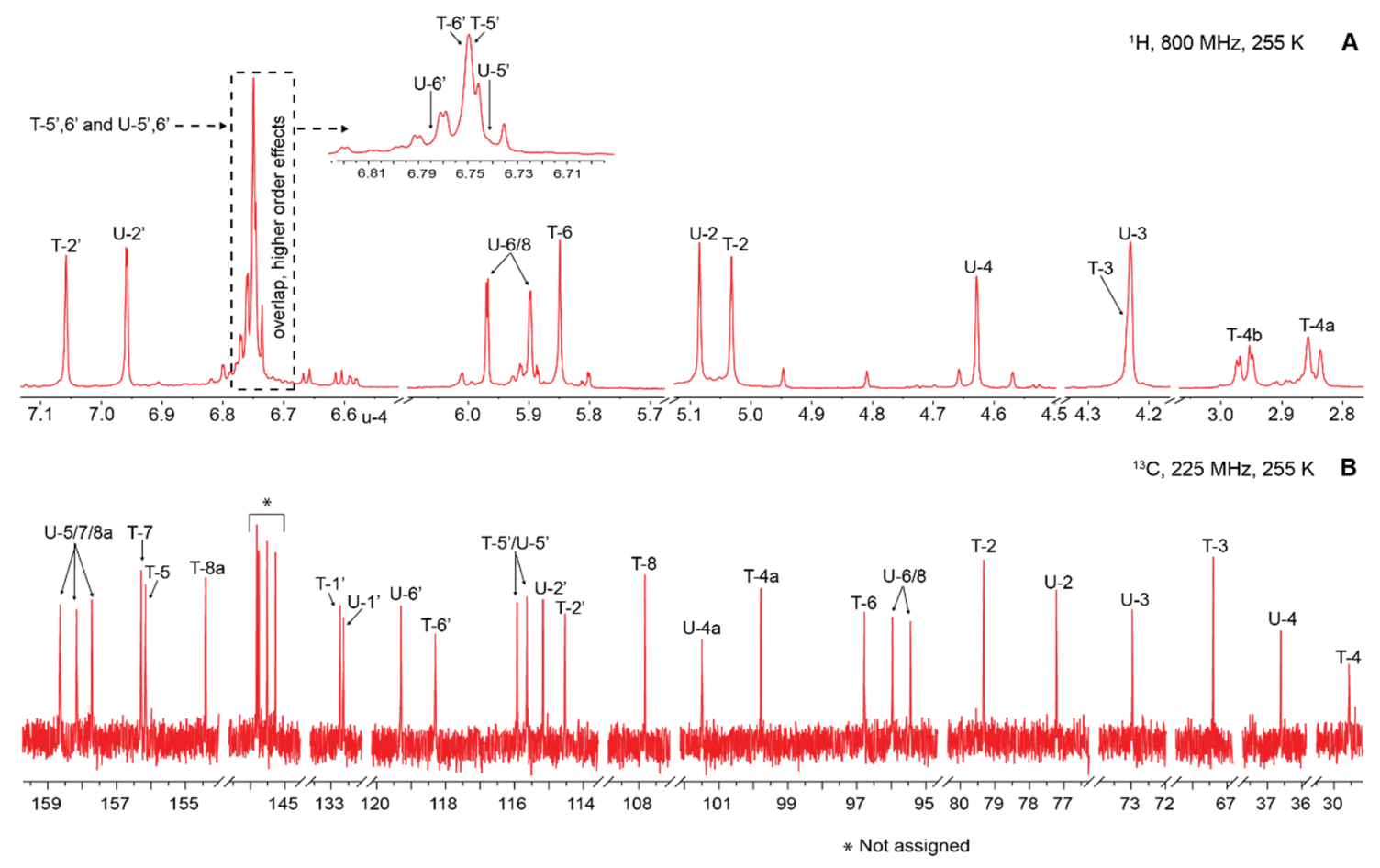

Figure S3 A: ${ }^{1} \mathrm{H}$ (A) and ${ }^{13} \mathrm{C}$ NMR (B) spectra for 5 in methanol- $d 4$. H-5'/6' from the B-ring AMX spin system of $\mathbf{5}$ are involved in higher order effect (dotted region; Figure 6 main text).

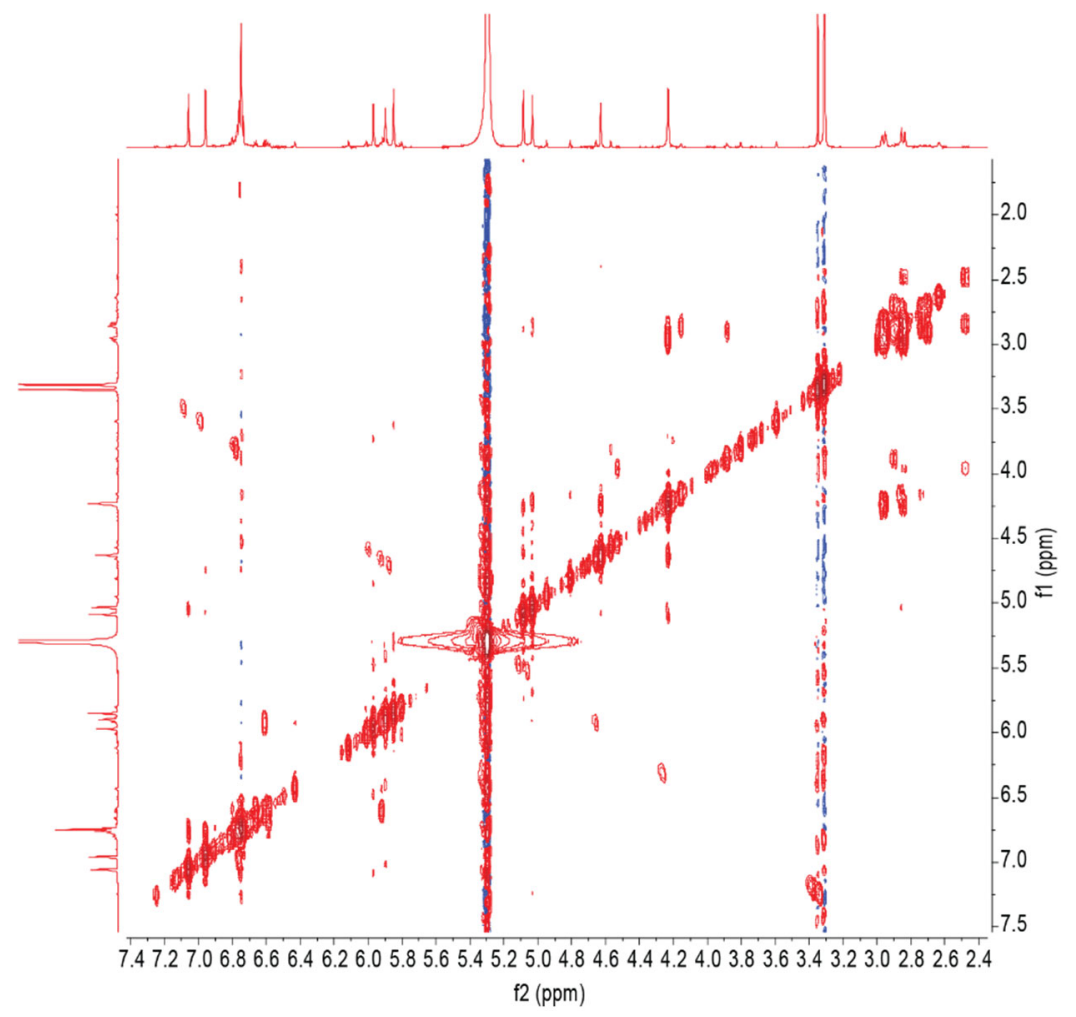

Figure S3 B: Compound 5, COSY spectrum 


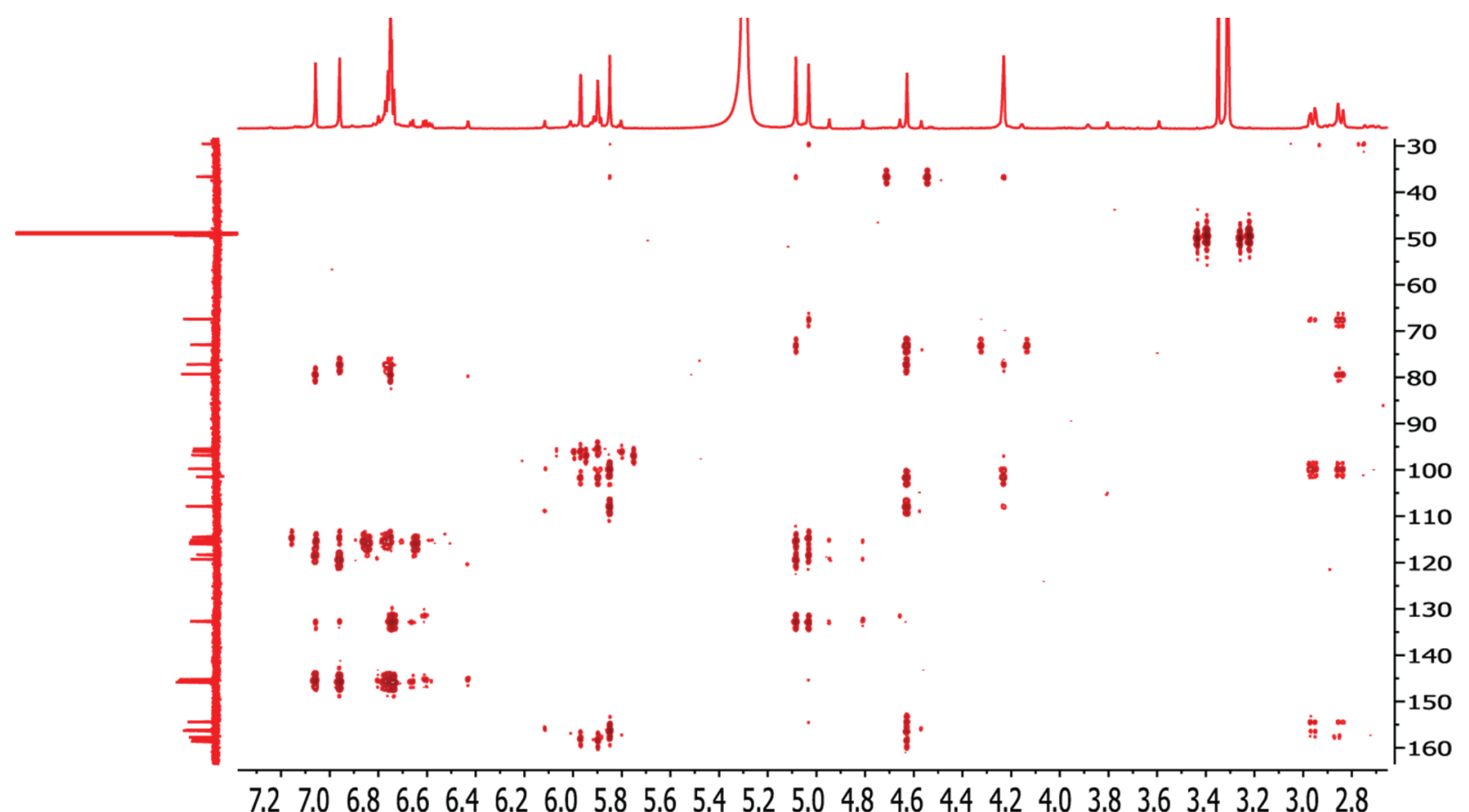

Figure S3 C: Compound 5, HMBC spectrum

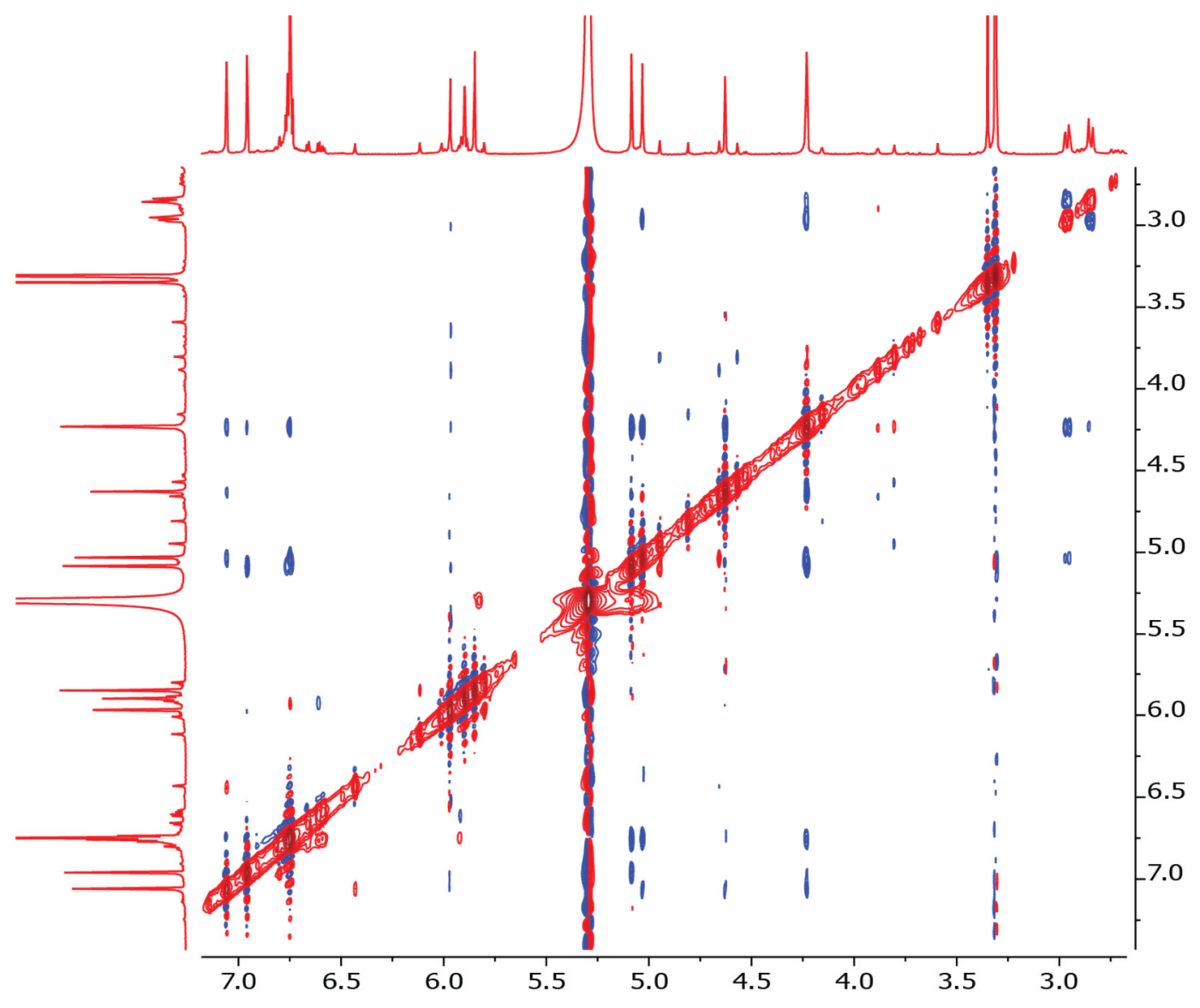

Figure S3 D: Compound 5, ROESY spectrum 


\section{S4}
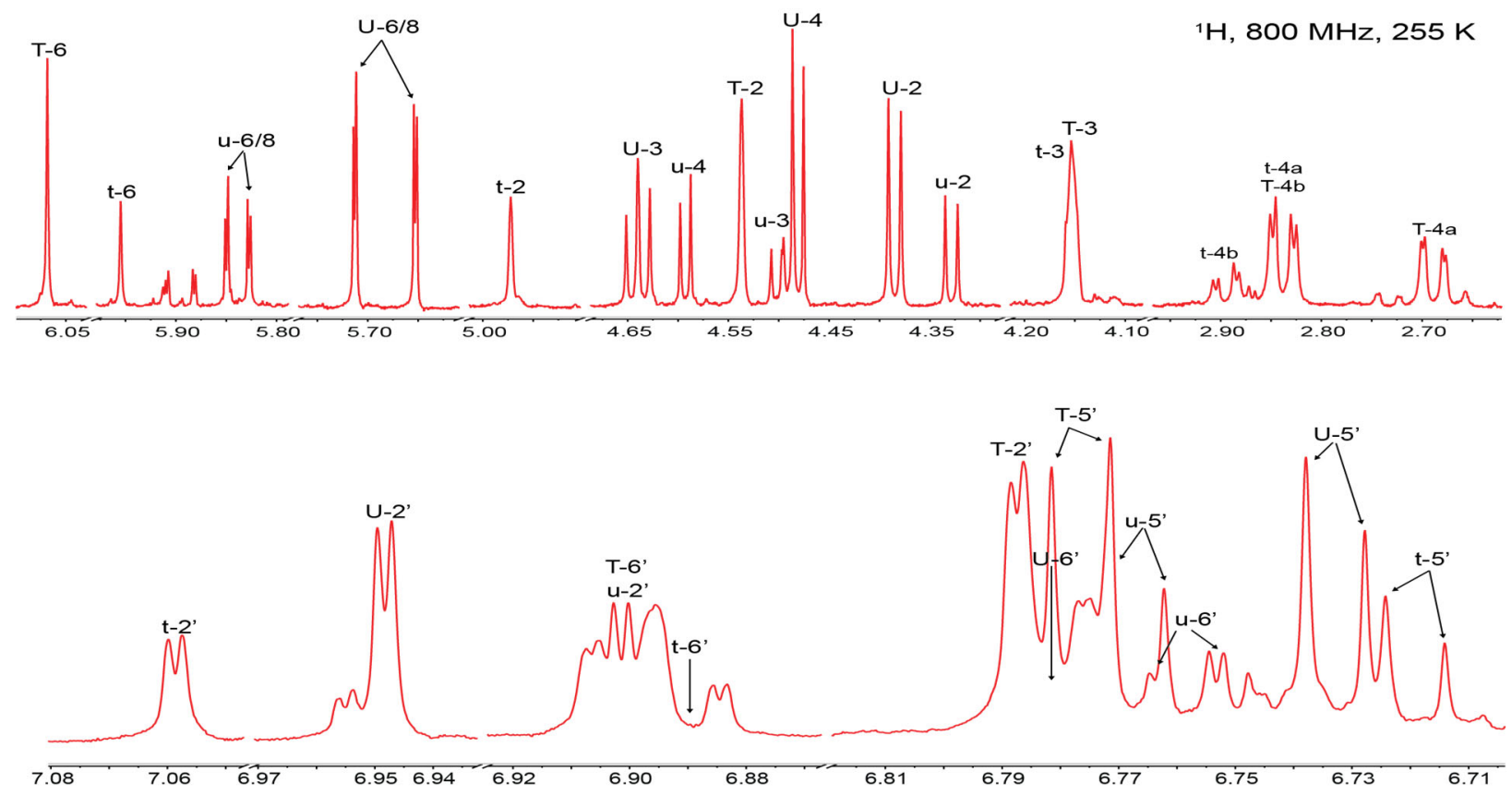

Figure S4 A: ${ }^{1} \mathrm{H}$ NMR spectrum of compound 6 in methanol- $d_{4}$. The spectrum was acquired on an $800 \mathrm{MHz}$ NMR at $255 \mathrm{~K}$. The $\delta^{1} \mathrm{H}$ of both the major and minor rotamers of 6 are assigned; $\mathrm{U} / \mathrm{T}$ and $\mathrm{u} / \mathrm{t}$ : upper and terminal unit of major and minor rotamers.

${ }^{13} \mathrm{C}, 225 \mathrm{MHz}, 255 \mathrm{~K}$

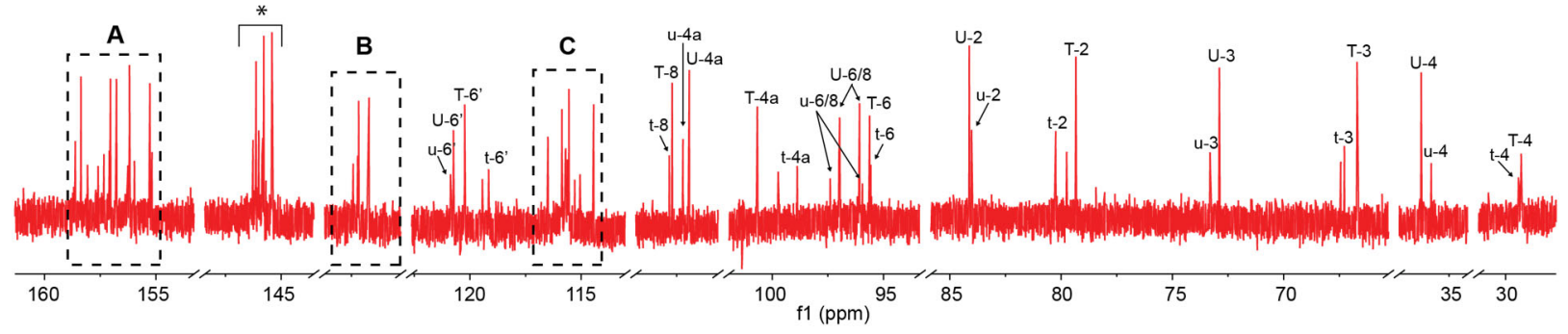

A

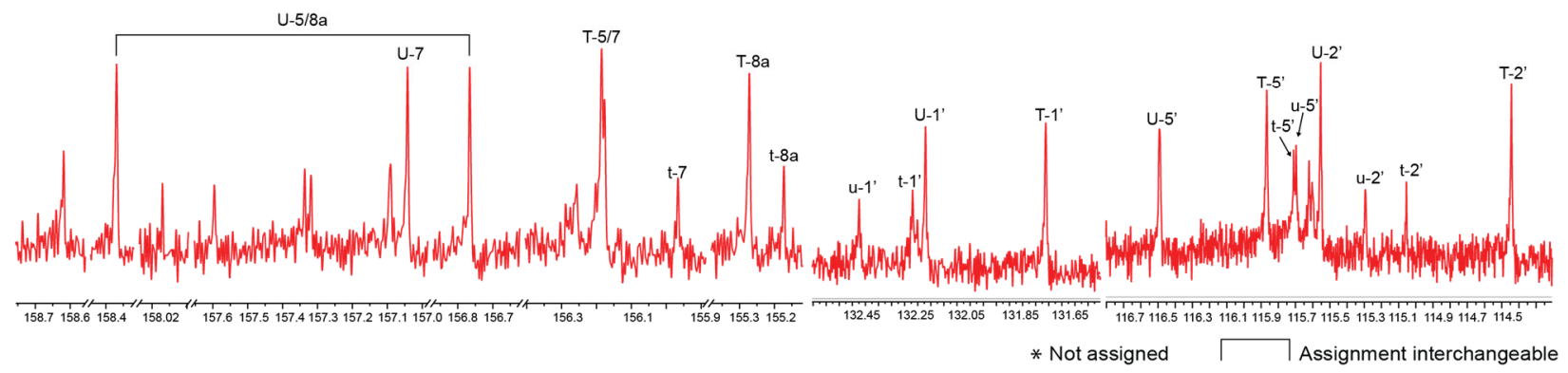

Figure S4 B: ${ }^{13} \mathrm{C}$ NMR spectrum of 6 in methanol- $d_{4}$ Panels A, B and C: Expansions of the Aring quaternary carbon region, B-ring quaternary $\mathrm{C}$-1' carbons and carbons from the B-ring AMX spin system; U/T and $\mathrm{u} / \mathrm{t}$ : upper and terminal unit of major and minor rotamers. 


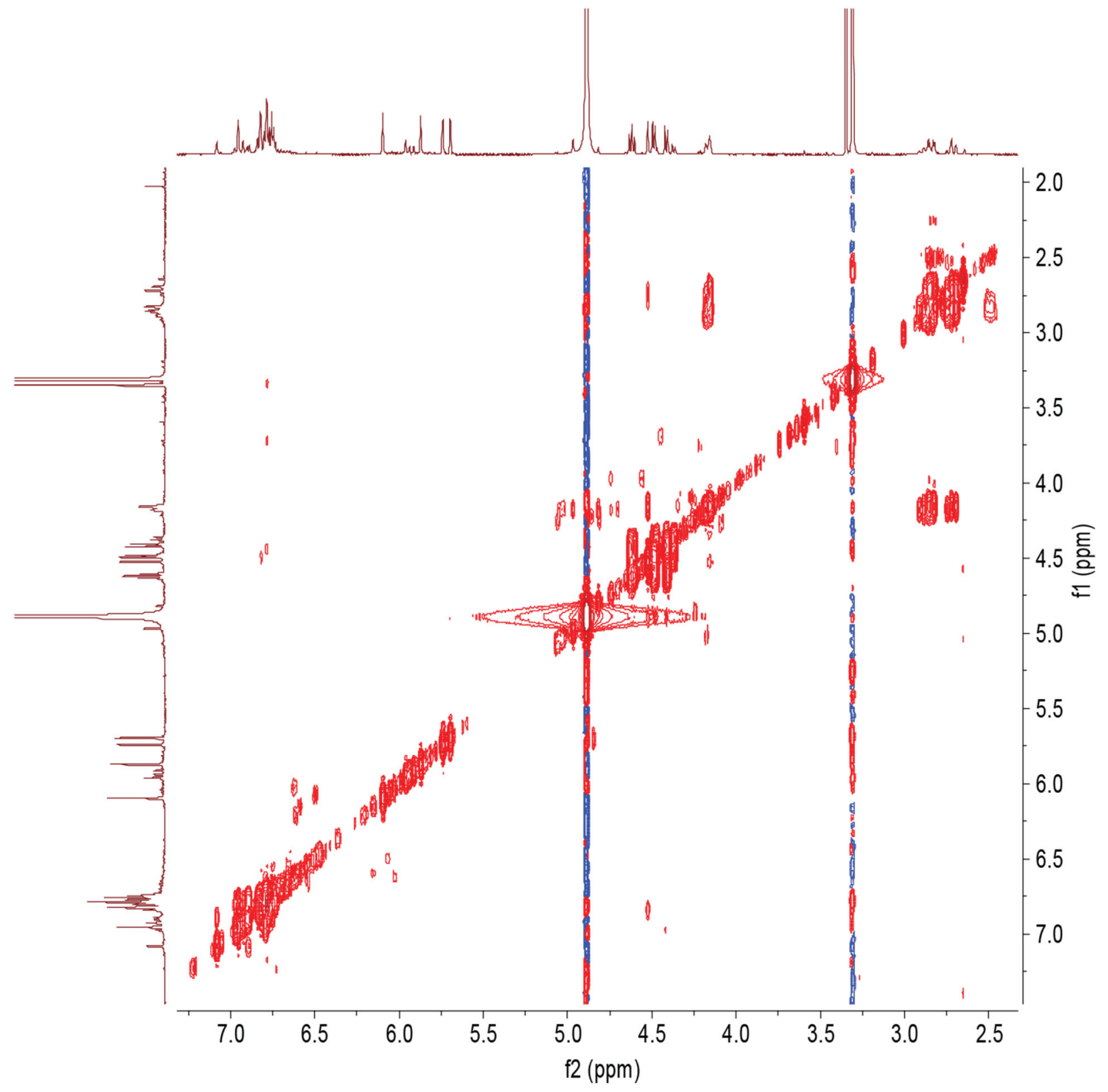

Figure S4 C: Compound 6, COSY spectrum 


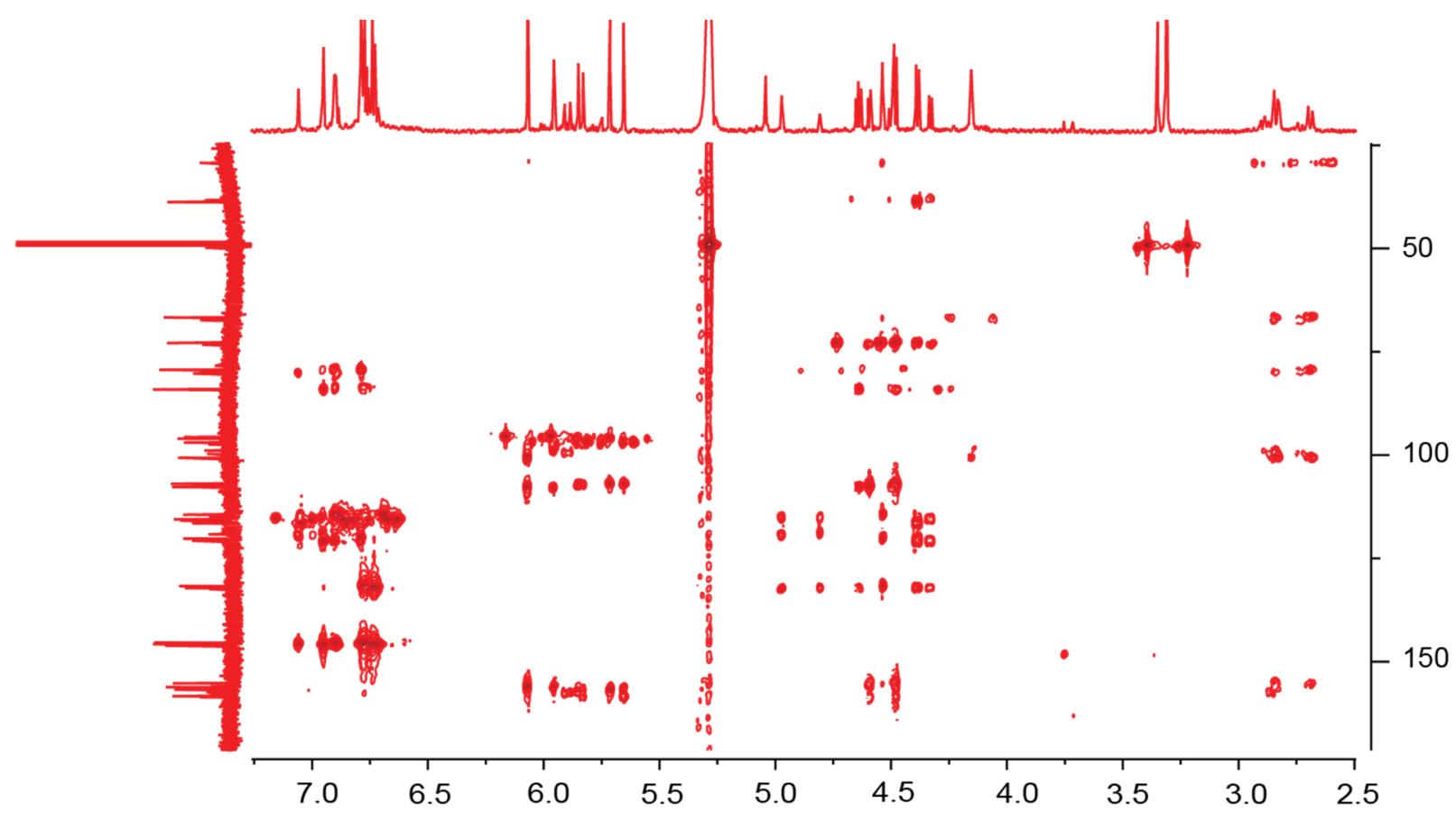

Figure S4 D: Compound 6, HMBC spectrum

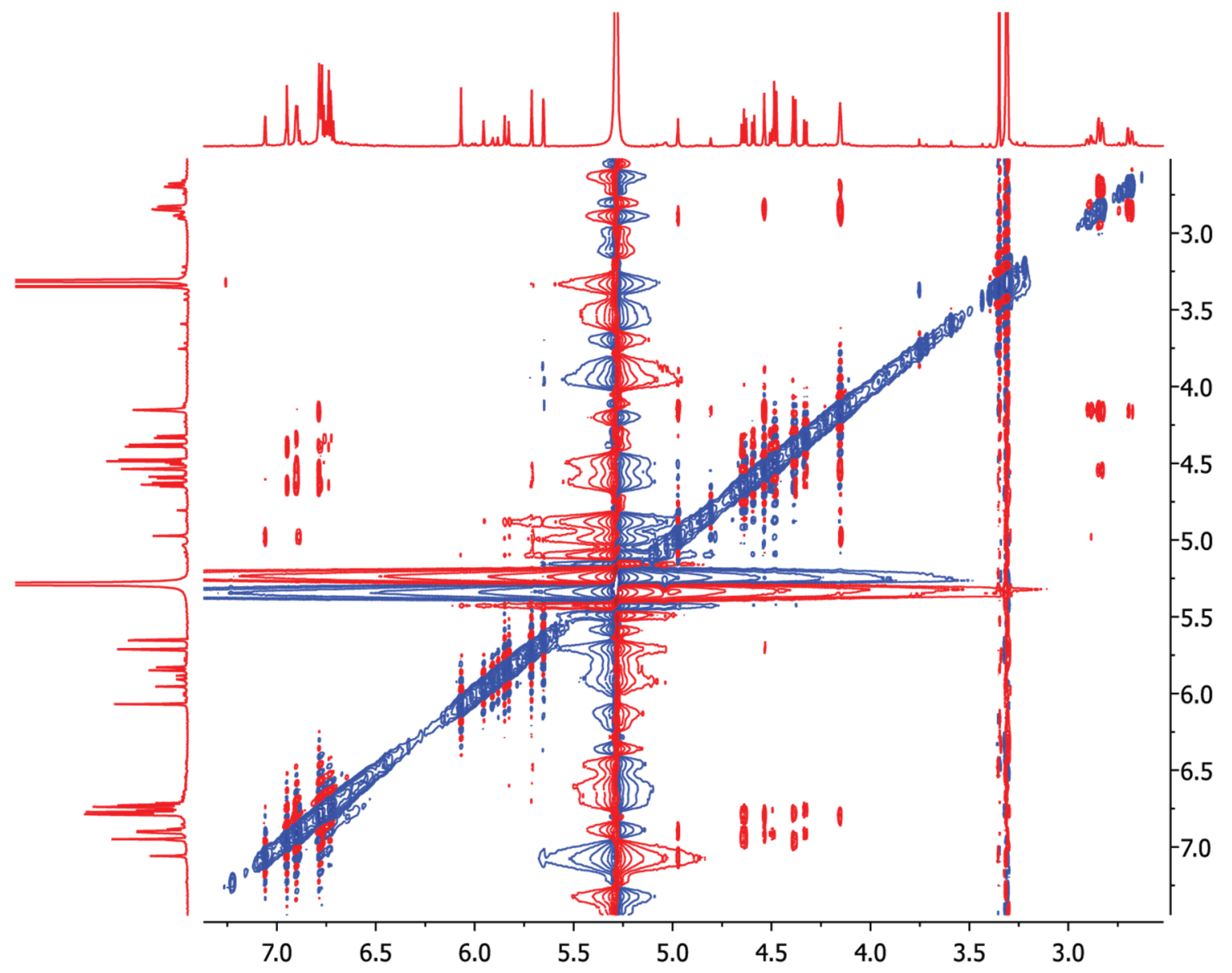

Figure S4 E: Compound 6, ROESY spectrum 


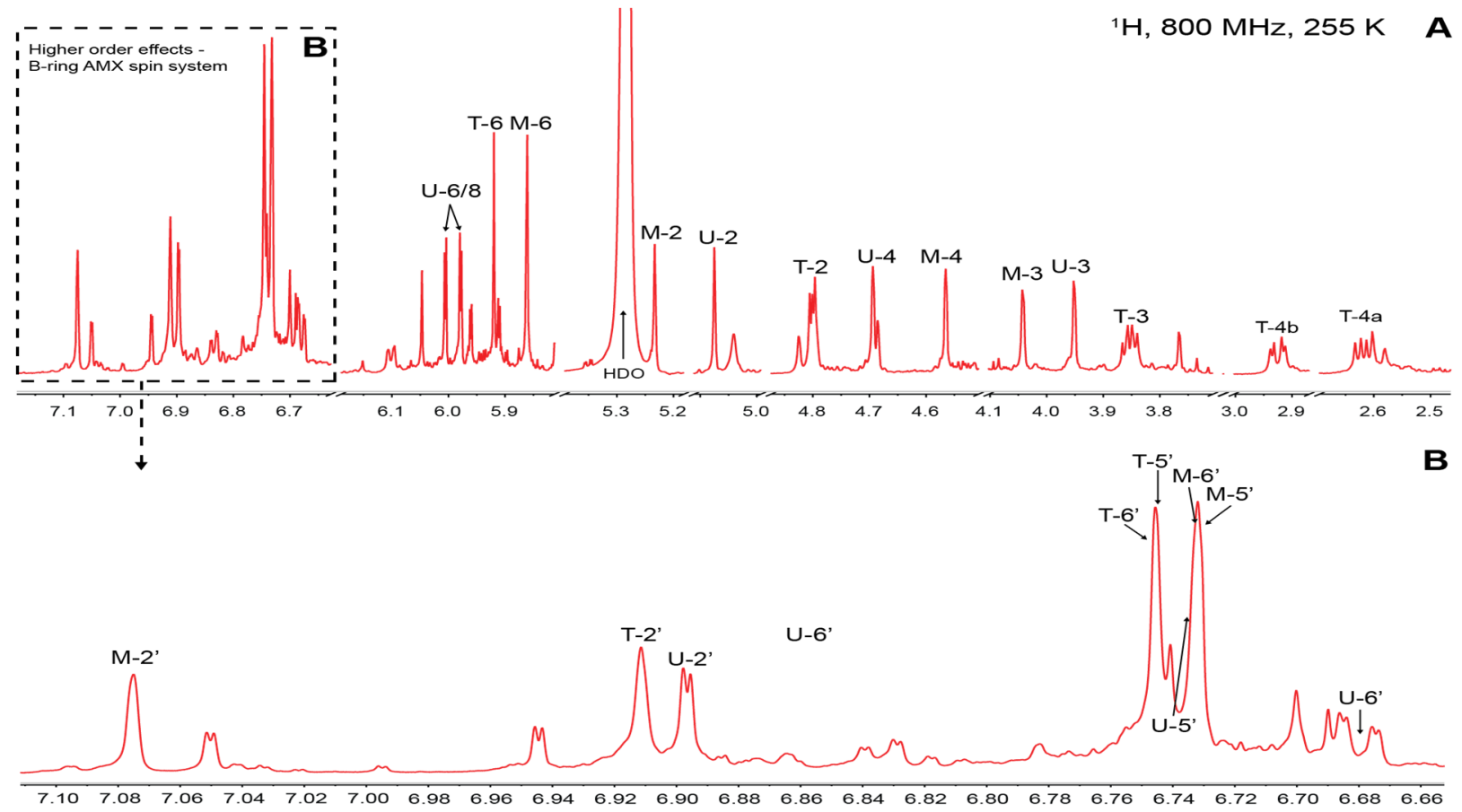

Figure S5 A: ${ }^{1} \mathrm{H}$ NMR spectrum of compound 9 in methanol- $d 4$. Panel B: Expansion of B-ring AMX spin system region affected by higher order effects.
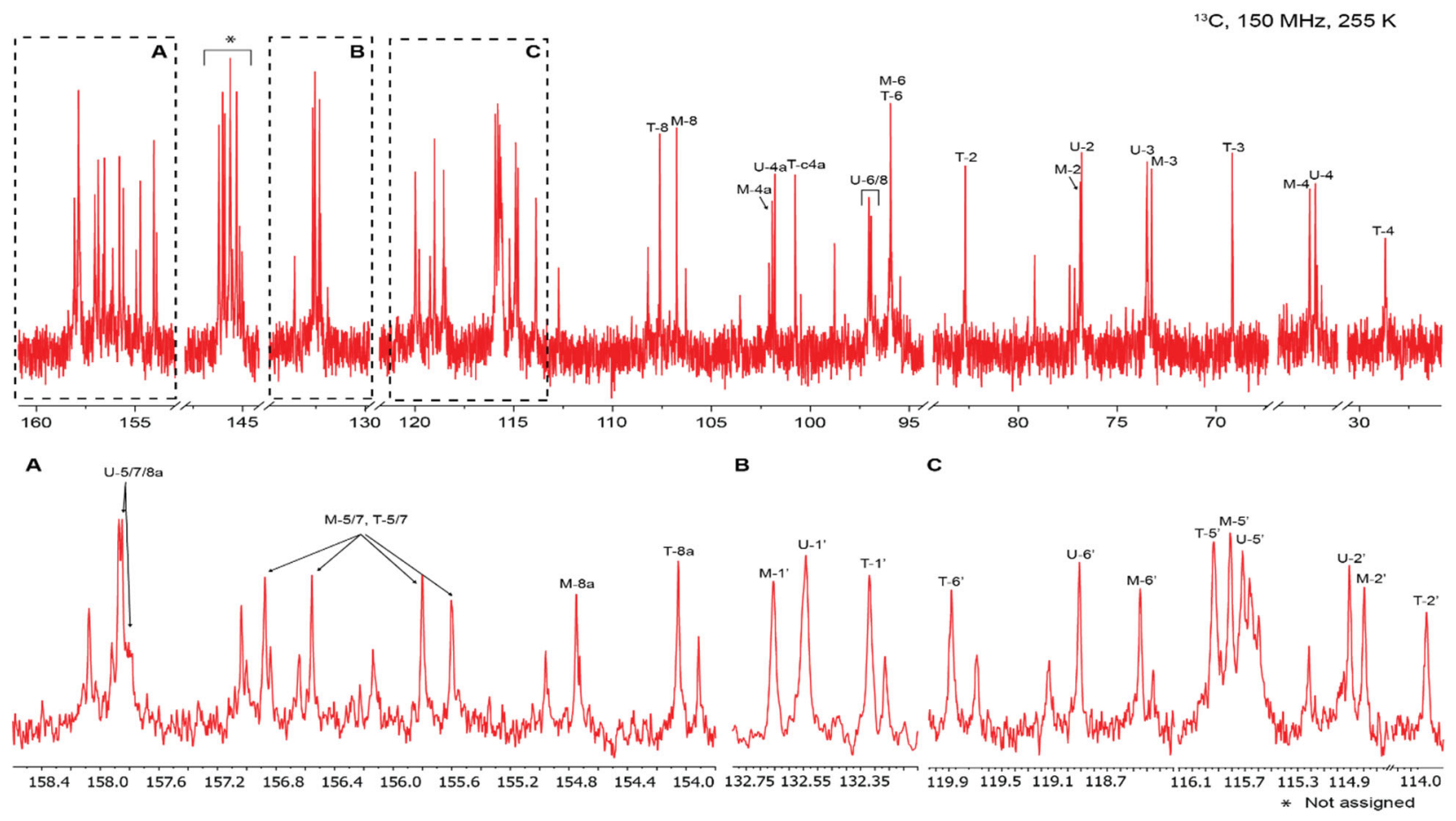

Figure S5 B: ${ }^{13} \mathrm{C}$ NMR spectrum of 9 acquired at $255 \mathrm{~K}$ in methanol- $d_{4}$ on a $600 \mathrm{MHz}$ NMR (150 MHz carbon channel frequency) instrument. Panel A: A-ring quaternary carbons; panel B: B-ring quaternary carbons $\mathrm{C}-1$ position; panel C: B-ring AMX spin system carbon assignments. 


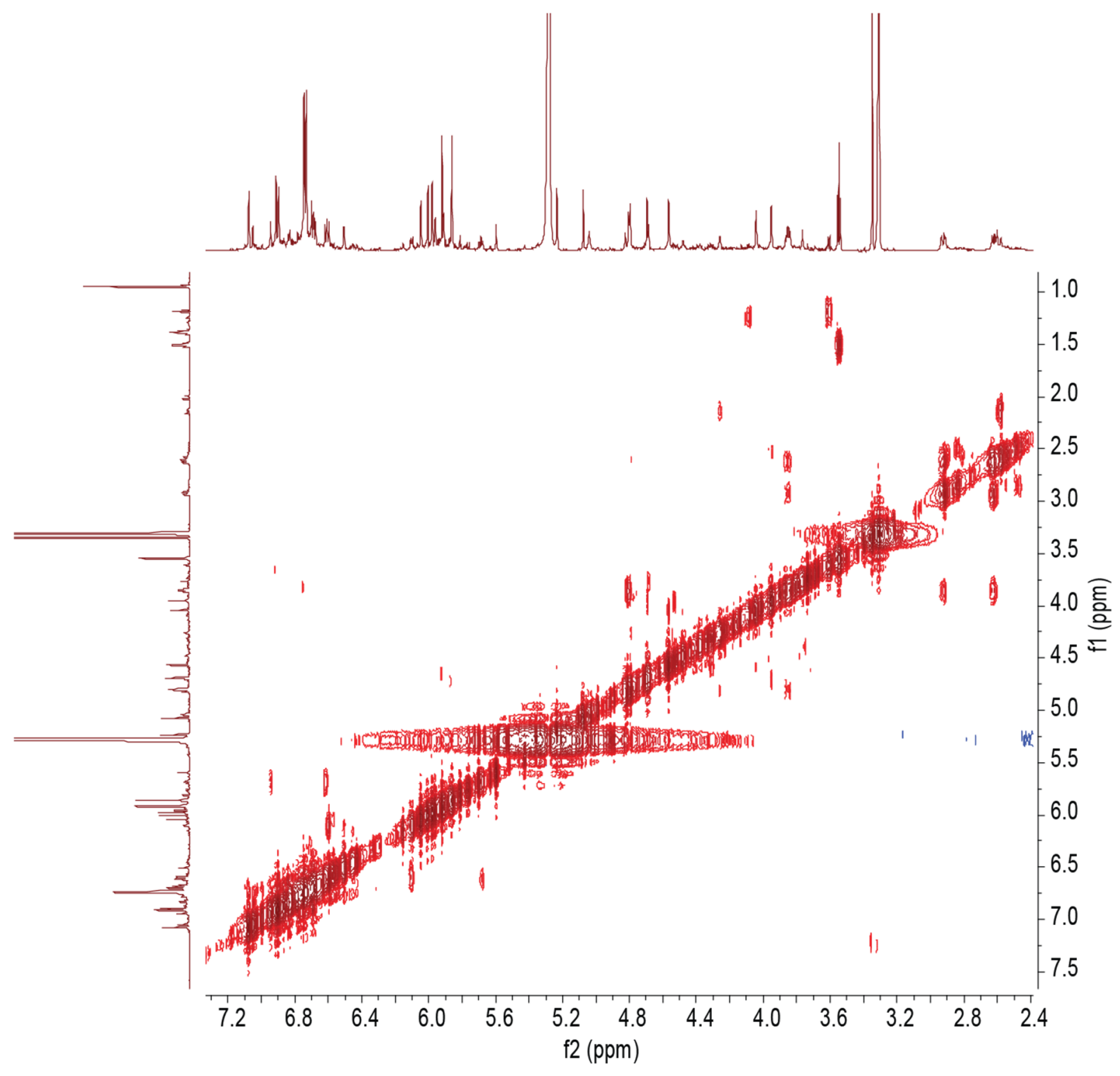

Figure S5 C: Compound 9, COSY spectrum 


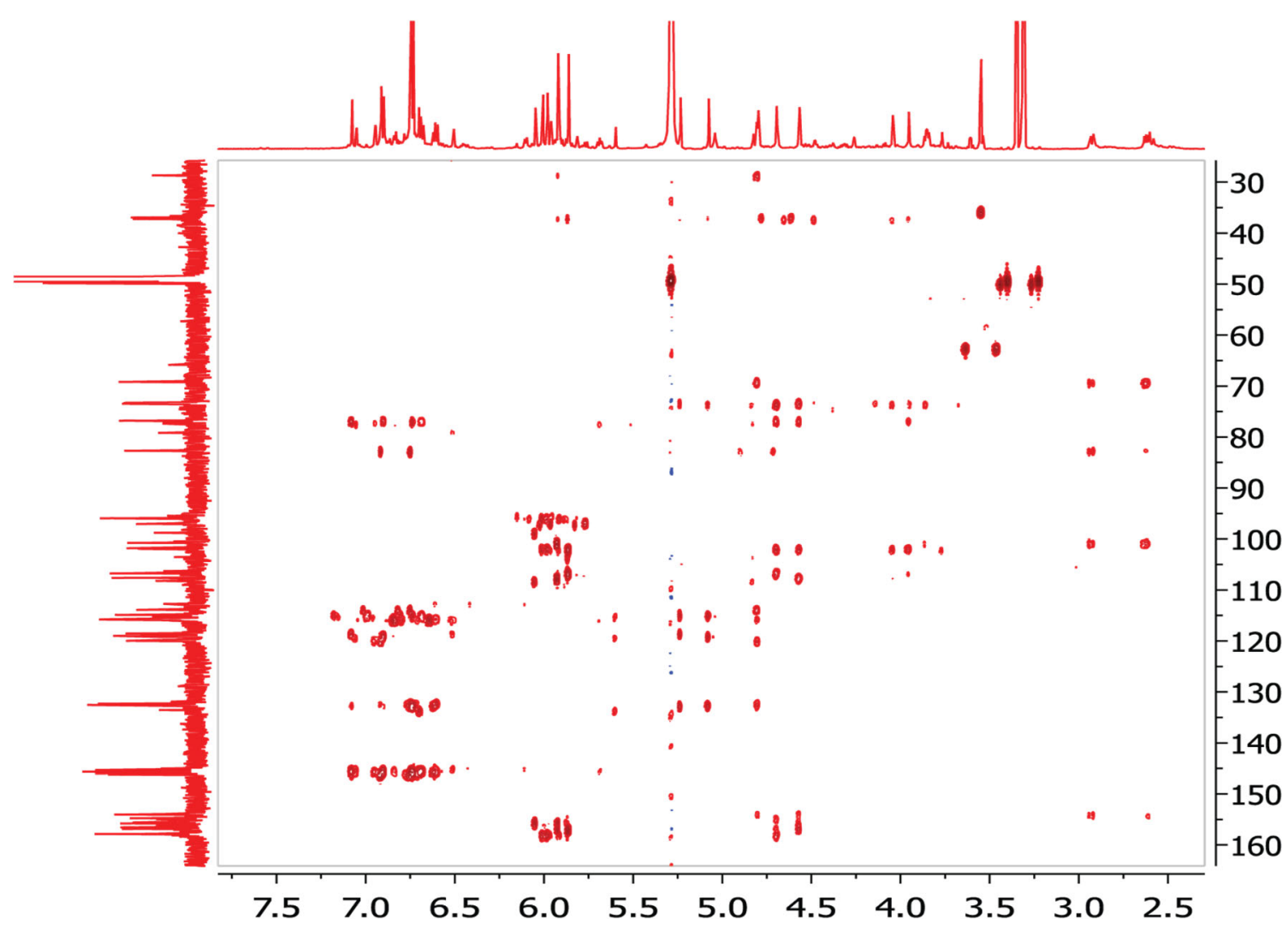

Figure S5 D: Compound 9, HMBC spectrum

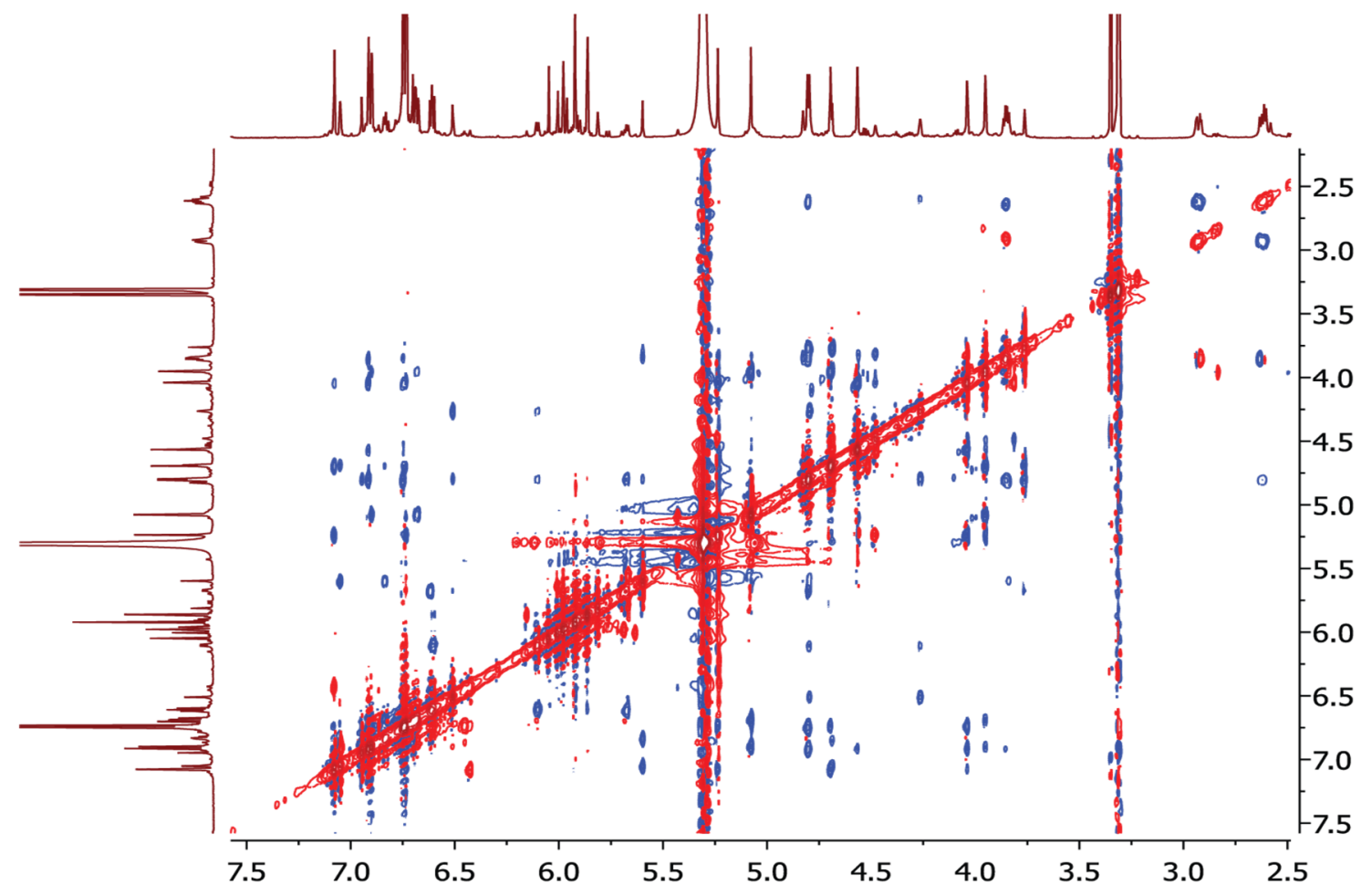

Figure S5 E: Compound 9, ROESY spectrum 


\title{
S6
}

\section{HiFSA parameter sets for compounds 1-9}

\author{
PMS file for 1 \\ NMR-data: c:luserslvmuserldesktop|rasika_dentalprojectlpab1|perch
}

\#\$ Date 11.10.2016; Time 20: 5:51 perch.pms

ACTIVE SPECIES:1H

CHEMICAL SHIFTS(PPM):

PROTON $2 *$ SPIN $=1$ SPECIES $=1 \mathrm{H} \quad$ POPULATION $(\mathrm{Y})=0.84233$

$\mathrm{H} 31 / 14.9548491 * 1 * 1$ STAT $=Y$ PRED $=4.431$ RANGE $=0.375 \mathrm{WIDTH}(\mathrm{Y})=3.606 \mathrm{RESP}(\mathrm{Y})=1.0000 \mathrm{HSQC}=\mathrm{C} 31$

$\mathrm{H} 32 / 14.1618081 * 1 * 1$ STAT $=Y$ PRED $=3.892$ RANGE $=0.250 \mathrm{WIDTH}(\mathrm{Y})=2.647 \mathrm{RESP}(\mathrm{Y})=1.0000 \mathrm{HSQC}=\mathrm{C} 32$

$\mathrm{H} 8 / 15.1000801 * 1 * 1$ STAT=Y PRED $=5.187$ RANGE=0.349 WIDTH(Y)=3.239 RESP $(\mathrm{Y})=1.0000 \mathrm{HSQC}=\mathrm{C} 8$

$\mathrm{H} 9 / 13.9398281 * 1 * 1$ STAT $=\mathrm{Y}$ PRED $=4.186 \mathrm{RANGE}=0.425 \mathrm{WIDTH}(\mathrm{N})=4.013 \mathrm{RESP}(\mathrm{Y})=1.0000 \mathrm{HSQC}=\mathrm{C} 9$

$\mathrm{H} 33 \mathrm{~A} / 12.6020691 * 1 * 1$ STAT $=\mathrm{Y}$ PRED $=2.483 \mathrm{RANGE}=0.220 \mathrm{WIDTH}(\mathrm{Y})=4.516 \operatorname{RESP}(\mathrm{Y})=1.0000 \mathrm{HSQC}=\mathrm{C} 33$

$\mathrm{H} 33 \mathrm{~B} / 12.5928581 * 1 * 1$ STAT $=Y$ PRED $=2.811$ RANGE $=0.184 \mathrm{WIDTH}(\mathrm{Y})=3.904 \operatorname{RESP}(\mathrm{Y})=1.0000 \mathrm{HSQC}=\mathrm{C} 33$

$\mathrm{H} 25 / 15.8384651 * 1 * 1$ STAT $=Y$ PRED $=5.878$ RANGE $=0.500 \mathrm{WIDTH}(\mathrm{Y})=1.444 \mathrm{RESP}(\mathrm{Y})=1.0000 \mathrm{HSQC}=\mathrm{C} 25$

$\mathrm{H} 4 / 15.9292561 * 1 * 1$ STAT=Y PRED $=5.969$ RANGE= $0.270 \mathrm{WIDTH}(\mathrm{Y})=2.035 \mathrm{RESP}(\mathrm{Y})=1.0000 \mathrm{HSQC}=\mathrm{C} 4$

$\mathrm{H} 1 / 15.9476321 * 1 * 1$ STAT=Y PRED $=5.796$ RANGE= $0.420 \mathrm{WIDTH}(\mathrm{Y})=2.163 \mathrm{RESP}(\mathrm{Y})=1.0000 \mathrm{HSQC}=\mathrm{C} 1$

$\mathrm{H} 10 / 14.6635541 * 1 * 1$ STAT=Y PRED $=4.590$ RANGE $=0.490 \mathrm{WIDTH}(\mathrm{Y})=3.672 \operatorname{RESP}(\mathrm{Y})=1.0000 \mathrm{HSQC}=\mathrm{C} 10$

$\mathrm{H} 14 / 16.6847571 * 1 * 1$ STAT $=Y$ PRED $=6.630$ RANGE $=0.150$ WIDTH $(\mathrm{Y})=2.546$ RESP $(\mathrm{Y})=1.0000 \mathrm{HSQC}=\mathrm{C} 14$

$\mathrm{H} 15 / 16.7006961{ }^{*} 1{ }^{*} 1$ STAT=Y PRED $=6.720$ RANGE $=0.150 \mathrm{WIDTH}(\mathrm{Y})=1.943 \mathrm{RESP}(\mathrm{Y})=1.0000 \mathrm{HSQC}=\mathrm{C} 15$

$\mathrm{H} 17 / 16.8749631 * 1 * 1$ STAT $=\mathrm{Y}$ PRED $=6.968$ RANGE $=0.150 \mathrm{WIDTH}(\mathrm{Y})=2.484 \operatorname{RESP}(\mathrm{Y})=1.0000 \mathrm{HSQC}=\mathrm{C} 17$

$\mathrm{H} 35 / 16.8828951 * 1 * 1$ STAT=Y PRED $=6.806$ RANGE $=0.320 \mathrm{WIDTH}(\mathrm{Y})=2.827 \mathrm{RESP}(\mathrm{Y})=1.0000 \mathrm{HSQC}=\mathrm{C} 35$

$\mathrm{H} 36 / 16.7155221 * 1 * 1$ STAT $=Y$ PRED $=6.672$ RANGE $=0.200 \mathrm{WIDTH}(\mathrm{Y})=1.871 \mathrm{RESP}(\mathrm{Y})=1.0000 \mathrm{HSQC}=\mathrm{C} 36$

$\mathrm{H} 39 / 16.8557231 * 1 * 1$ STAT=Y PRED= 6.663 RANGE=0.290 WIDTH(Y)=2.516 RESP $(Y)=1.0000 \mathrm{HSQC}=\mathrm{C} 39$

UNK01 $2 *$ SPIN $=1$ SPECIES $=1 \mathrm{H} \quad$ POPULATION $(\mathrm{Y})=0.15767$

$2-\mathrm{H} 1 / 2 \quad 3.9258241 * 1 * 1$ STAT $=\mathrm{Y}$ PRED $=3.926 \mathrm{RANGE}=0.120 \mathrm{WIDTH}(\mathrm{Y})=1.880 \mathrm{RESP}(\mathrm{Y})=1.0000$

$2-\mathrm{H} 4 / 220.0000021 * 1 * 1$ STAT $=Y$ PRED $=10.926$ RANGE $=0.120 \mathrm{WIDTH}(\mathrm{Y})=1.600 \mathrm{RESP}(\mathrm{Y})=1.0000$

$2-\mathrm{H} 6 / 26.8652031 * 1 * 1$ STAT=Y PRED $=6.860 \mathrm{RANGE}=0.120 \mathrm{WIDTH}(\mathrm{Y})=3.180 \operatorname{RESP}(\mathrm{Y})=1.0000$

\begin{tabular}{|c|c|c|c|c|c|}
\hline \multicolumn{6}{|c|}{ OUPLING CONSTANTS(HZ): } \\
\hline J53_54 & 5.3990 & $\mathrm{~J} \mathrm{H} 31$ & $\mathrm{H} 32$ & STAT $=Y$ & PRED $=9.745$ RANGE $=2.000$ \\
\hline J54_57 & 5.5919 & $\mathrm{~J} \mathrm{H} 32$ & $\mathrm{H} 33 \mathrm{~A}$ & STAT $=Y$ & $Y$ PRED $=11.291$ RANGE $=3.130$ \\
\hline $\mathrm{J} 54-58$ & 5.4552 & J H32 & $\mathrm{H} 33 \mathrm{~B}$ & STAT $=Y$ & Y PRED $=4.493$ RANGE $=2.000$ \\
\hline 56 & 0.0462 & $\mathrm{~J} \mathrm{H} 8$ & $\mathrm{H} 9 \mathrm{~S}$ & $S T A T=Y P$ & PREL \\
\hline $\mathrm{J} 56 \quad 62$ & 1.5160 & $\mathrm{~J} \mathrm{H9}$ & $\mathrm{H} 10$ & STAT $=Y \quad F$ & PRED $=2.8$ \\
\hline $\mathrm{J} 57^{-} 58$ & -15.5953 & $\mathrm{~J} \mathrm{H} 33$ & ЗА H33E & B STAT $=$ & $=\mathrm{N}$ \\
\hline J60_61 & 2.0517 & $\mathrm{JH}$ & $\mathrm{H} 1$ & $S T A T=N P$ & PRED $=2$ \\
\hline J63_64 & 8.2300 & $\mathrm{~J} \mathrm{H} 14$ & H15 & $S T A T=Y$ & PRED $=8.186$ RANGE $=1.000$ \\
\hline J63_65 & 76 & $\mathrm{JH}$ & $\mathrm{H} 17$ & $S T A T=Y$ & PRED = \\
\hline 65 & 0.0417 & J H15 & $\mathrm{H} 17$ & $\mathrm{STAT}=\mathrm{Y}$ & RANGE $=1.000$ \\
\hline 67 & & & $\mathrm{H} 36$ & STAT $=Y$ & \\
\hline & & & & STAT $=Y$ & \\
\hline & & & & $\mathrm{TAT}=\mathrm{Y}$ & 000 \\
\hline & & & & & .0 \\
\hline
\end{tabular}

CONTROL PARAMETERS:

Solvent $=$ none $($ def. $99 \%$ enriched $)$

$1.000=$ Concentration $($ vol $\%$, def $=1.0 \%)$

$0.00100000=$ Minimum line-intensity

$0.00100000=$ Diagonalization criterium (not in use)

$800.20420000=\mathrm{FIELD}(1 \mathrm{H}, \mathrm{MHz})$, used to transform shifts to ppms

$12.26555493=$ Left frequency $(\mathrm{ppm})$

$-1.68174729=$ Right frequency $(\mathrm{ppm})$

$10.000=$ Acquisition time (s, for QMTLS)

$0.000=$ Line-width (for modes D, P \& T, 0=use defaults)

$0.072200001=$ Data-point resolution $(\mathrm{Hz})$

$9.681=$ GAUSSIAN (\%, $0=$ use default from INF)

$4.507=$ Dispersion contribution (\%, 0=use default from INF)

$0.00000000=$ Decoupling frequency (for DORES) 


\section{PMS file for 2}

NMR-data: c:Iuserslvmuserldesktoplrasika_dentalprojectlngd02/perch

\#\$® Date 8.10.2016; Time 15:14:25 perch.pms

\section{ACTIVE SPECIES:1H}

CHEMICAL SHIFTS(PPM):

Major $2 *$ SPIN $=1$ SPECIES $=1 \mathrm{H} \quad$ POPULATION $(\mathrm{Y})=0.69055$

$\mathrm{H} 31 / 1$ 4.764179 $1 * 1 * 1$ STAT=Y PRED $=4.630$ RANGE $=0.295 \mathrm{WIDTH}(\mathrm{Y})=2.645 \operatorname{RESP}(\mathrm{Y})=1.0000 \mathrm{HSQC}=\mathrm{C} 31$

$\mathrm{H} 32 / 13.8511221 * 1 * 1$ STAT=Y PRED $=3.858$ RANGE $=0.269$ WIDTH $(\mathrm{Y})=2.472$ RESP $(\mathrm{Y})=1.0000 \mathrm{HSQC}=\mathrm{C} 32$

$\mathrm{H} 8 / 15.0404491 * 1 * 1$ STAT $=Y$ PRED $=4.881$ RANGE $=0.250 \mathrm{WIDTH}(\mathrm{Y})=2.558 \operatorname{RESP}(\mathrm{Y})=1.0000 \mathrm{HSQC}=\mathrm{C} 8$

$\mathrm{H} 9 / 13.9620031 * 1 * 1$ STAT=Y PRED $=4.066$ RANGE $=0.415 \mathrm{WIDTH}(\mathrm{Y})=2.692 \operatorname{RESP}(\mathrm{Y})=1.0000 \mathrm{HSQC}=\mathrm{C} 9$

$\mathrm{H} 33 \mathrm{~A} / 12.9134041^{*} 1^{*} 1$ STAT $=\mathrm{Y}$ PRED $=2.803$ RANGE $=0.165 \mathrm{WIDTH}(\mathrm{Y})=3.494 \mathrm{RESP}(\mathrm{Y})=1.0000 \mathrm{HSQC}=\mathrm{C} 33$

$\mathrm{H} 33 \mathrm{~B} / 12.5989821 * 1 * 1$ STAT=Y PRED $=2.527$ RANGE $=0.185 \mathrm{WIDTH}(\mathrm{Y})=3.147 \operatorname{RESP}(\mathrm{Y})=1.0000 \mathrm{HSQC}=\mathrm{C} 33$

$\mathrm{H} 25 / 15.8604151 * 1 * 1$ STAT $=Y$ PRED $=5.895$ RANGE $=0.525$ WIDTH $(Y)=1.373 \operatorname{RESP}(Y)=1.0000 \mathrm{HSQC}=\mathrm{C} 25$

$\mathrm{H} 4 / 15.95645911^{*} 1 * 1$ STAT=Y PRED= 6.012 RANGE=0.195 WIDTH(Y)=1.918 RESP $(\mathrm{Y})=1.0000 \mathrm{HSQC}=\mathrm{C} 4$

$\mathrm{H} 1 / 15.8992961 * 1 * 1$ STAT $=\mathrm{Y}$ PRED $=5.870 \mathrm{RANGE}=0.235 \mathrm{WIDTH}(\mathrm{Y})=1.667 \operatorname{RESP}(\mathrm{Y})=1.0000 \mathrm{HSQC}=\mathrm{C} 1$

$\mathrm{H} 10 / 14.4814201 * 1 * 1$ STAT=Y PRED $=4.525 \mathrm{RANGE}=0.479 \mathrm{WIDTH}(\mathrm{Y})=2.493 \operatorname{RESP}(\mathrm{Y})=1.0000 \mathrm{HSQC}=\mathrm{C} 10$

$\mathrm{H} 14 / 16.6704201 * 1 * 1$ STAT $=Y$ PRED $=6.934$ RANGE $=0.150 \mathrm{WIDTH}(\mathrm{Y})=2.266 \operatorname{RESP}(\mathrm{Y})=1.0000 \mathrm{HSQC}=\mathrm{C} 14$

$\mathrm{H} 15 / 16.7415961 * 1 * 1$ STAT=Y PRED $=6.708$ RANGE $=0.150 \mathrm{WIDTH}(\mathrm{Y})=1.623 \operatorname{RESP}(\mathrm{Y})=1.0000 \mathrm{HSQC}=\mathrm{C} 15$

$\mathrm{H} 17 / 16.9006891 * 1 * 1$ STAT $=Y$ PRED $=6.687$ RANGE $=0.190 \mathrm{WIDTH}(\mathrm{Y})=1.912 \mathrm{RESP}(\mathrm{Y})=1.0000 \mathrm{HSQC}=\mathrm{C} 17$

$\mathrm{H} 35 / 16.7254561 * 1 * 1$ STAT=Y PRED $=6.647$ RANGE $=0.150 \mathrm{WIDTH}(\mathrm{Y})=2.108 \mathrm{RESP}(\mathrm{Y})=1.0000 \mathrm{HSQC}=\mathrm{C} 35$

$\mathrm{H} 36 / 16.7251931 * 1 * 1$ STAT=Y PRED $=6.699$ RANGE $=0.185 \mathrm{WIDTH}(\mathrm{Y})=2.173 \mathrm{RESP}(\mathrm{Y})=1.0000 \mathrm{HSQC}=\mathrm{C} 36$

$\mathrm{H} 39 / 16.8659821 * 1 * 1$ STAT=Y PRED=6.748 RANGE=0.735 WIDTH(Y)=2.244 RESP(Y)=1.0000 HSQC $=\mathrm{C} 39$

Minor $2 *$ SPIN $=1$ SPECIES $=1 \mathrm{H} \quad$ POPULATION $(\mathrm{Y})=0.30945$

U31 / $24.3970251 * 1 * 1$ STAT=Y PRED $=4.630$ RANGE $=0.295 \mathrm{WIDTH}(\mathrm{Y})=2.877 \mathrm{RESP}(\mathrm{Y})=1.0000 \mathrm{HSQC}=\mathrm{C} 31$

U32 / $23.7826171 * 1 * 1$ STAT=Y PRED $=3.858$ RANGE $=0.269$ WIDTH $(Y)=2.651$ RESP $(Y)=1.0000 \mathrm{HSQC}=\mathrm{C} 32$

U8 $/ 25.1030791 * 1 * 1$ STAT $=Y$ PRED $=4.881$ RANGE $=0.250 \mathrm{WIDTH}(\mathrm{Y})=2.542 \operatorname{RESP}(\mathrm{Y})=1.0000 \mathrm{HSQC}=\mathrm{C} 8$

U9 / $23.8010681 * 1 * 1$ STAT=Y PRED $=4.066$ RANGE $=0.415 \mathrm{WIDTH}(\mathrm{Y})=4.019 \mathrm{RESP}(\mathrm{Y})=1.0000 \mathrm{HSQC}=\mathrm{C} 33$

$\mathrm{U} 33 \mathrm{~A} / 22.6192751 * 1 * 1$ STAT $=\mathrm{Y}$ PRED $=2.803 \mathrm{RANGE}=0.165 \mathrm{WIDTH}(\mathrm{Y})=4.072 \mathrm{RESP}(\mathrm{Y})=1.0000 \mathrm{HSQC}=\mathrm{C} 33$

U33B/ $22.4902001 * 1 * 1$ STAT=Y PRED $=2.527$ RANGE $=0.185 \operatorname{WIDTH}(Y)=3.373 \operatorname{RESP}(Y)=1.0000 \mathrm{HSQC}=\mathrm{C} 33$

$\mathrm{U} 25 / 26.0679121 * 1 * 1$ STAT $=Y$ PRED $=5.895 \mathrm{RANGE}=0.525 \mathrm{WIDTH}(\mathrm{Y})=1.868 \operatorname{RESP}(\mathrm{Y})=1.0000 \mathrm{HSQC}=\mathrm{C} 25$

U4 $/ 25.856767 \quad 1 * 1 * 1$ STAT=Y PRED= 6.012 RANGE=0.195 WIDTH(Y)=2.049 RESP $(\mathrm{Y})=1.0000 \mathrm{HSQC}=\mathrm{C} 4$

$\mathrm{U} 1 / 25.6401461 * 1 * 1$ STAT $=\mathrm{Y}$ PRED $=5.870 \mathrm{RANGE}=0.235 \mathrm{WIDTH}(\mathrm{Y})=3.424 \operatorname{RESP}(\mathrm{Y})=1.0000 \mathrm{HSQC}=\mathrm{C} 1$

$\mathrm{U} 10 / 24.5759381 * 1 * 1$ STAT=Y PRED $=4.525 \mathrm{RANGE}=0.479 \mathrm{WIDTH}(\mathrm{Y})=2.916 \mathrm{RESP}(\mathrm{Y})=1.0000 \mathrm{HSQC}=\mathrm{C} 10$

$\mathrm{U} 14 / 26.6607471 * 1 * 1$ STAT $=\mathrm{Y}$ PRED $=6.934 \mathrm{RANGE}=0.150 \mathrm{WIDTH}(\mathrm{Y})=1.946 \mathrm{RESP}(\mathrm{Y})=1.0000 \mathrm{HSQC}=\mathrm{C} 14$

$\mathrm{U} 15 / 26.6812121 * 1 * 1$ STAT=Y PRED $=6.708$ RANGE $=0.150 \mathrm{WIDTH}(\mathrm{Y})=1.747 \mathrm{RESP}(\mathrm{Y})=1.0000 \mathrm{HSQC}=\mathrm{C} 15$

$\mathrm{U} 17 / 26.8504511 * 1 * 1$ STAT $=Y$ PRED $=6.687$ RANGE $=0.190 \mathrm{WIDTH}(\mathrm{Y})=2.032 \mathrm{RESP}(\mathrm{Y})=1.0000 \mathrm{HSQC}=\mathrm{C} 17$

$\mathrm{U} 35 / 26.14530611^{*} 1 * 1$ STAT=Y PRED $=6.647$ RANGE $=0.150 \mathrm{WIDTH}(\mathrm{Y})=2.187 \mathrm{RESP}(\mathrm{Y})=1.0000 \mathrm{HSQC}=\mathrm{C} 35$

$\mathrm{U} 36 / 26.6142791 * 1 * 1$ STAT $=Y$ PRED $=6.699 \mathrm{RANGE}=0.185 \mathrm{WIDTH}(\mathrm{Y})=1.805 \mathrm{RESP}(\mathrm{Y})=1.0000 \mathrm{HSQC}=\mathrm{C} 36$

U39 / $26.4510041 * 1 * 1$ STAT=Y PRED= 6.748 RANGE=0.735 WIDTH(Y)=2.093 RESP $(Y)=1.0000 \mathrm{HSQC}=\mathrm{C} 39$

COUPLING CONSTANTS(HZ):

J53_54 7.4036 J H31 H32 STAT=Y PRED $=9.745$ RANGE $=2.000$

$\mathrm{J} 5457 \quad 5.4418 \quad \mathrm{~J} \mathrm{H} 32$ H33A STAT $=Y$ PRED $=4.119$ RANGE $=2.000$

J54_58 8.2401 J H32 H33B STAT=Y PRED= 11.731 RANGE $=2.000$

J55_56 $0.9801 \quad \mathrm{~J} \mathrm{H} 8$ H9 STAT $=Y$ PRED $=2.713$ RANGE $=2.000$

J56_62 1.9348 J H9 H10 STAT=Y PRED $=3.902$ RANGE $=3.530$

J57_58 $-16.0843 \quad \mathrm{~J} \mathrm{H} 33 \mathrm{~A}$ H33B STAT $=Y$ PRED $=-15.791$ RANGE $=1.340$

J60_61 2.0736 J H4 H1 STAT=Y PRED $=2.184$ RANGE $=1.000$

J63 $64 \quad 8.1595 \quad \mathrm{~J} \mathrm{H} 14$ H15 STAT $=Y$ PRED $=8.186$ RANGE $=1.000$

J63_65 2.0291 J H14 H17 STAT=Y PRED $=2.083$ RANGE $=1.000$

J64_65 $0.0350 \quad \mathrm{~J} \mathrm{H} 15 \quad \mathrm{H} 17 \quad \mathrm{STAT}=\mathrm{Y}$ PRED $=0.500$ RANGE $=1.000$

J66_67 9.3097 J H35 H36 STAT=Y PRED= 8.185 RANGE= 1.000

J66_68 $1.0430 \mathrm{~J} \mathrm{H} 35$ H39 STAT $=Y$ PRED $=2.083$ RANGE $=1.000$

J67_68 $0.9527 \mathrm{~J} \mathrm{H} 36$ H39 STAT $=Y$ PRED $=0.500$ RANGE $=1.000$

$\mathrm{J} 15 \overline{3} \_546.5827 \mathrm{~J}$ U31 U32 STAT $=Y$ PRED $=9.745$ RANGE $=2.000$

J154_57 5.3240 J U32 U33A STAT=Y PRED $=4.119$ RANGE $=2.000$

J154_58 7.0942 J U32 U33B STAT=Y PRED $=11.731$ RANGE $=2.000$

J155_56 1.0968 J U8 U9 STAT $=Y$ PRED $=2.713$ RANGE $=2.000$

J156_62 1.8337 J U9 U10 STAT=Y PRED $=3.902$ RANGE $=3.530$

J157_58 $-16.2593 \quad \mathrm{~J} U 33 \mathrm{~A}$ U33B STAT $=Y$ PRED $=-15.791$ RANGE $=1.340$

J160_61 2.1694 J U4 U1 STAT $=Y$ PRED $=2.184$ RANGE $=1.000$

J163_64 8.2271 J U14 U15 STAT $=Y$ PRED $=8.186$ RANGE $=1.000$

J163_65 2.0316 J U14 U17 STAT=Y PRED $=2.083$ RANGE $=1.000$ 
J164_65 $0.0464 \quad \mathrm{~J} U 15$ U17 STAT $=Y$ PRED $=0.500$ RANGE $=1.000$

J166_67 8.1321 J U35 U36 STAT=Y PRED $=8.185$ RANGE $=1.000$

J166_68 2.0525 J U35 U39 STAT $=Y$ PRED $=2.083$ RANGE $=1.000$

J167_68 0.0212 J U36 U39 STAT=Y PRED $=0.500$ RANGE $=1.000$

CONTROL PARAMETERS:

Solvent $=$ none $($ def. $99 \%$ enriched $)$

$1.000=$ Concentration $($ vol $\%$, def $=1.0 \%)$

$0.00100000=$ Minimum line-intensity

$0.00100000=$ Diagonalization criterium (not in use)

$800.20420000=$ FIELD $(1 \mathrm{H}, \mathrm{MHz})$, used to transform shifts to ppms

$12.25040517=$ Left frequency $(\mathrm{ppm})$

$-1.69687254=$ Right frequency $(\mathrm{ppm})$

$0.000=$ Acquisition time (s, for QMTLS)

$1.357=$ Line-width (for modes D, P \& T, 0=use defaults)

$0.068650002=$ Data-point resolution $(\mathrm{Hz})$

$14.043=$ GAUSSIAN $(\%, 0=$ use default from INF)

$5.544=$ Dispersion contribution (\%, 0=use default from INF)

$0.00000000=$ Decoupling frequency (for DORES)

\section{PMS file for 3}

NMR-data: c:luserslvmuserldesktop|rasika_dentalprojectlngd03|perch

\#\$œ Date 19.10.2016; Time 10:13:19 perch.pms

ACTIVE SPECIES:1H

CHEMICAL SHIFTS(PPM):

Major $2 *$ SPIN $=1$ SPECIES $=1 \mathrm{H} \quad$ POPULATION $(\mathrm{Y})=0.51227$

$\mathrm{H} 2 / 15.7410651 * 1 * 1$ STAT=Y PRED $=5.955$ RANGE $=0.175 \mathrm{WIDTH}(\mathrm{Y})=1.142 \mathrm{RESP}(\mathrm{Y})=1.0000 \mathrm{HSQC}=\mathrm{C} 2$

$\mathrm{H} 6 / 15.5308301 * 1 * 1$ STAT=Y PRED $=5.881$ RANGE= $0.150 \mathrm{WIDTH}(\mathrm{Y})=1.200 \mathrm{RESP}(\mathrm{Y})=1.0000 \mathrm{HSQC}=\mathrm{C} 6$

$\mathrm{H} 7 / 14.3672831 * 1 * 1$ STAT=Y PRED $=4.483$ RANGE=0.495 WIDTH $(\mathrm{Y})=1.349 \mathrm{RESP}(\mathrm{Y})=1.0000 \mathrm{HSQC}=\mathrm{C} 7$

$\mathrm{H} 8 / 14.38637311^{*}{ }^{* 1}$ STAT=Y PRED $=4.371$ RANGE= $0.480 \mathrm{WIDTH}(\mathrm{Y})=1.575 \operatorname{RESP}(\mathrm{Y})=1.0000 \mathrm{HSQC}=\mathrm{C} 8$

$\mathrm{H} 9 / 14.1997331 * 1 * 1$ STAT=Y PRED $=4.798$ RANGE= $0.310 \mathrm{WIDTH}(\mathrm{Y})=1.545 \mathrm{RESP}(\mathrm{Y})=1.0000 \mathrm{HSQC}=\mathrm{C} 9$

$\mathrm{H} 14 / 1 \quad 6.040651 \quad 1 * 1 * 1$ STAT $=\mathrm{Y}$ PRED $=6.004$ RANGE $=0.500 \mathrm{WIDTH}(\mathrm{Y})=1.737 \mathrm{RESP}(\mathrm{Y})=1.0000 \mathrm{HSQC}=\mathrm{C} 14$

$\mathrm{H} 18 \mathrm{~A} / 12.9018461 * 1 * 1 \mathrm{STAT}=\mathrm{Y}$ PRED $=2.660 \mathrm{RANGE}=0.235 \mathrm{WIDTH}(\mathrm{Y})=2.494 \operatorname{RESP}(\mathrm{Y})=1.0000 \mathrm{HSQC}=\mathrm{C} 18$

$\mathrm{H} 18 \mathrm{~B} / 12.41950611^{*} 1$ STAT $=\mathrm{Y}$ PRED $=2.586$ RANGE $=0.315 \mathrm{WIDTH}(\mathrm{Y})=2.586 \mathrm{RESP}(\mathrm{Y})=1.0000 \mathrm{HSQC}=\mathrm{C} 18$

$\mathrm{H} 19 / 13.9016301 * 1 * 1$ STAT $=\mathrm{Y}$ PRED $=4.044 \mathrm{RANGE}=0.290 \mathrm{WIDTH}(\mathrm{Y})=1.909 \operatorname{RESP}(\mathrm{Y})=1.0000 \mathrm{HSQC}=\mathrm{C} 19$

$\mathrm{H} 20 / 14.0934021 * 1 * 1$ STAT $=\mathrm{Y}$ PRED $=4.673$ RANGE $=0.390 \mathrm{WIDTH}(\mathrm{Y})=1.808 \mathrm{RESP}(\mathrm{Y})=1.0000 \mathrm{HSQC}=\mathrm{C} 20$

$\mathrm{H} 26 / 16.5779291 * 1 * 1$ STAT $=\mathrm{Y}$ PRED $=6.756 \mathrm{RANGE}=0.150 \mathrm{WIDTH}(\mathrm{Y})=1.629 \mathrm{RESP}(\mathrm{Y})=1.0000 \mathrm{HSQC}=\mathrm{C} 26$

$\mathrm{H} 27 / 16.7397561{ }^{*}{ }^{*} 1$ STAT $=\mathrm{Y}$ PRED $=6.751$ RANGE $=0.150 \mathrm{WIDTH}(\mathrm{Y})=1.280 \mathrm{RESP}(\mathrm{Y})=1.0000 \mathrm{HSQC}=\mathrm{C} 27$

$\mathrm{H} 30 / 16.7833851 * 1 * 1$ STAT $=\mathrm{Y}$ PRED $=6.812$ RANGE $=0.505 \mathrm{WIDTH}(\mathrm{Y})=1.618 \operatorname{RESP}(\mathrm{Y})=1.0000 \mathrm{HSQC}=\mathrm{C} 30$

$\mathrm{H} 32 / 16.6572161{ }^{*} 1 * 1$ STAT $=\mathrm{Y}$ PRED $=6.563 \mathrm{RANGE}=0.150 \mathrm{WIDTH}(\mathrm{Y})=1.573 \mathrm{RESP}(\mathrm{Y})=1.0000 \mathrm{HSQC}=\mathrm{C} 32$

$\mathrm{H} 33 / 16.8039881 * 1 * 1$ STAT $=\mathrm{Y}$ PRED $=6.746$ RANGE $=0.175 \mathrm{WIDTH}(\mathrm{Y})=1.328 \mathrm{RESP}(\mathrm{Y})=1.0000 \mathrm{HSQC}=\mathrm{C} 33$

$\mathrm{H} 36 / 16.7908551 * 1 * 1$ STAT $=\mathrm{Y}$ PRED $=6.697$ RANGE $=0.495 \mathrm{WIDTH}(\mathrm{Y})=1.522 \mathrm{RESP}(\mathrm{Y})=1.0000 \mathrm{HSQC}=\mathrm{C} 36$

Minor $2 *$ SPIN $=1$ SPECIES $=1 \mathrm{H} \quad$ POPULATION $(\mathrm{Y})=0.48773$

U2 / 2 5.829504 $1 * 1 * 1$ STAT=Y PRED= 5.955 RANGE $=0.175 \mathrm{WIDTH}(\mathrm{Y})=1.254 \mathrm{RESP}(\mathrm{Y})=1.0000 \mathrm{HSQC}=\mathrm{C} 2$

U6 / $25.7960751 * 1 * 1$ STAT=Y PRED= 5.881 RANGE=0.150 WIDTH(Y)=1.233 RESP $(Y)=1.0000 \mathrm{HSQC}=\mathrm{C} 6$

U7 / $24.4750961 * 1 * 1$ STAT $=Y$ PRED $=4.483$ RANGE $=0.495 \mathrm{WIDTH}(\mathrm{Y})=1.647 \mathrm{RESP}(\mathrm{Y})=1.0000 \mathrm{HSQC}=\mathrm{C} 7$

U8 / $24.4385431 * 1 * 1$ STAT=Y PRED $=4.371$ RANGE= $0.480 \mathrm{WIDTH}(\mathrm{Y})=1.666 \mathrm{RESP}(\mathrm{Y})=1.0000 \mathrm{HSQC}=\mathrm{C} 8$

U9 / $24.3047531 * 1 * 1$ STAT $=Y$ PRED $=4.798$ RANGE $=0.310 \mathrm{WIDTH}(\mathrm{Y})=1.657 \mathrm{RESP}(\mathrm{Y})=1.0000 \mathrm{HSQC}=\mathrm{C9}$

U14 / $25.9182781 * 1 * 1$ STAT=Y PRED $=6.004$ RANGE $=0.500$ WIDTH $(\mathrm{Y})=1.993$ RESP $(\mathrm{Y})=1.0000 \mathrm{HSQC}=\mathrm{C} 14$

$\mathrm{U} 18 \mathrm{~A} / 22.8495251 * 1 * 1$ STAT=Y PRED $=2.660 \mathrm{RANGE}=0.235 \mathrm{WIDTH}(\mathrm{Y})=3.537 \operatorname{RESP}(\mathrm{Y})=1.0000 \mathrm{HSQC}=\mathrm{C} 18$

$\mathrm{U} 18 \mathrm{~B} / 22.5398371 * 1 * 1$ STAT=Y PRED $=2.586 \mathrm{RANGE}=0.315 \mathrm{WIDTH}(\mathrm{Y})=2.911 \mathrm{RESP}(\mathrm{Y})=1.0000 \mathrm{HSQC}=\mathrm{C} 18$

$\mathrm{U} 19 / 23.9158141 * 1 * 1$ STAT $=Y$ PRED $=4.044$ RANGE $=0.290 \mathrm{WIDTH}(\mathrm{Y})=2.178 \mathrm{RESP}(\mathrm{Y})=1.0000 \mathrm{HSQC}=\mathrm{C} 19$

$\mathrm{U} 20 / 24.7362101 * 1 * 1$ STAT $=\mathrm{Y}$ PRED $=4.673 \mathrm{RANGE}=0.390 \mathrm{WIDTH}(\mathrm{Y})=2.300 \mathrm{RESP}(\mathrm{Y})=1.0000 \mathrm{HSQC}=\mathrm{C} 20$

$\mathrm{U} 26 / 26.7485251 * 1 * 1$ STAT $=\mathrm{Y}$ PRED $=6.756 \mathrm{RANGE}=0.150 \mathrm{WIDTH}(\mathrm{Y})=1.381 \mathrm{RESP}(\mathrm{Y})=1.0000 \mathrm{HSQC}=\mathrm{C} 26$

$\mathrm{U} 27 / 26.7205531 * 1 * 1$ STAT=Y PRED $=6.751$ RANGE $=0.150 \mathrm{WIDTH}(\mathrm{Y})=1.457 \mathrm{RESP}(\mathrm{Y})=1.0000 \mathrm{HSQC}=\mathrm{C} 27$

$\mathrm{U} 30 / 26.8915951 * 1 * 1$ STAT $=Y$ PRED $=6.812$ RANGE $=0.505 \mathrm{WIDTH}(\mathrm{Y})=1.339 \mathrm{RESP}(\mathrm{Y})=1.0000 \mathrm{HSQC}=\mathrm{C} 30$

U32 / $26.8978741 * 1 * 1$ STAT=Y PRED= 6.563 RANGE=0.150 WIDTH(Y)=1.975 RESP(Y)=1.0000 HSQC $=\mathrm{C} 32$

$\mathrm{U} 33 / 26.7711421 * 1 * 1$ STAT $=Y$ PRED $=6.746$ RANGE $=0.175 \mathrm{WIDTH}(\mathrm{Y})=1.508 \mathrm{RESP}(\mathrm{Y})=1.0000 \mathrm{HSQC}=\mathrm{C} 33$

U36 / $26.9168361 * 1 * 1$ STAT=Y PRED $=6.697$ RANGE $=0.495 \mathrm{WIDTH}(\mathrm{Y})=1.687 \mathrm{RESP}(\mathrm{Y})=1.0000 \mathrm{HSQC}=\mathrm{C} 36$

COUPLING CONSTANTS(HZ):

J43_44 2.3778 J H2 H6 STAT $=Y$ PRED $=2.184$ RANGE $=1.000$

$\mathrm{J} 4546 \quad 8.3623 \quad \mathrm{~J} \mathrm{H} 7 \quad \mathrm{H} 8 \quad \mathrm{STAT}=\mathrm{Y}$ PRED $=11.874$ RANGE $=3.580$

J46_47 9.8537 J H8 H9 STAT=Y PRED=9.762 RANGE $=2.800$ 


\author{
J49_50 -16.0717 J H18A H18B STAT=Y PRED $=-15.917$ RANGE $=1.280$ \\ J49_51 5.8323 J H18A H19 STAT $=Y$ PRED $=3.012$ RANGE $=2.000$ \\ J49_52 $0.6253 \mathrm{~J} \mathrm{H} 18 \mathrm{~A}$ H20 STAT $=Y \quad P R E D=2.471$ RANGE $=1.000$ \\ J50_51 8.7958 J H18B H19 STAT $=Y \quad P R E D=4.098$ RANGE $=2.000$ \\ J51_52 8.2200 J H19 H20 STAT $=Y$ PRED $=1.784$ RANGE $=2.000$ \\ J57_58 8.0923 J H26 H27 STAT $=Y$ PRED $=8.186$ RANGE $=1.000$ \\ J57_59 $1.9517 \mathrm{~J} \mathrm{H} 26$ H30 STAT=Y PRED $=2.083$ RANGE $=1.000$ \\ J58_59 $0.0067 \mathrm{~J} \mathrm{H} 27 \mathrm{H} 30 \quad$ STAT $=Y$ PRED $=0.500$ RANGE $=1.000$ \\ J60_61 8.0393 J H32 H33 STAT $=Y$ PRED $=8.185$ RANGE $=1.000$ \\ J60 $62 \quad 2.0338$ J H32 H36 STAT=Y PRED $=2.083$ RANGE $=1.000$ \\ J61_62 $0.0047 \mathrm{~J} H 33$ H36 STAT=Y PRED $=0.500$ RANGE $=1.000$ \\ $\mathrm{J} 143 \quad 442.3972 \quad \mathrm{~J}$ U2 U6 STAT $=Y$ PRED $=2.184 \mathrm{RANGE}=1.000$ \\ J145_46 8.2687 J U7 U8 STAT $=Y$ PRED $=11.874$ RANGE $=3.580$ \\ $\mathrm{J} 146 \quad 47 \quad 9.8954 \quad \mathrm{~J} \cup 8$ U9 $S T A T=Y$ PRED $=9.762$ RANGE $=2.800$ \\ J149_50 -15.8654 J U18A U18B STAT=Y PRED $=-15.917$ RANGE $=1.280$ \\ $\mathrm{J} 14951 \quad 5.3616 \quad \mathrm{~J}$ U18A U19 STAT $=Y \quad P R E D=3.012$ RANGE $=2.000$ \\ J149_52 0.6999 J U18A U20 STAT $=Y \quad P R E D=2.471$ RANGE $=1.000$ \\ $\mathrm{J} 150-518.1684 \mathrm{~J}$ U18B U19 STAT $=Y \quad P R E D=4.098$ RANGE $=2.000$ \\ J151_52 7.3153 J U19 U20 STAT $=Y$ PRED $=1.784$ RANGE $=2.000$ \\ J157_58 8.0766 J U26 U27 STAT $=Y$ PRED $=8.186$ RANGE $=1.000$ \\ J157_59 1.9889 J U26 U30 STAT $=Y$ PRED $=2.083$ RANGE $=1.000$ \\ J158_59 $0.3009 \quad \mathrm{~J} U 27$ U30 STAT $=Y$ PRED $=0.500$ RANGE $=1.000$ \\ J160_61 8.1007 J U32 U33 STAT $=Y$ PRED $=8.185$ RANGE $=1.000$ \\ J160_62 2.0218 J U32 U36 STAT $=Y$ PRED $=2.083$ RANGE $=1.000$ \\ J161_62 0.0604 J U33 U36 STAT $=Y$ PRED $=0.500$ RANGE $=1.000$ \\ CONTROL PARAMETERS: \\ Solvent $=$ none $($ def. $99 \%$ enriched $)$ \\ $1.000=$ Concentration $(v o l \%$, def $=1.0 \%)$ \\ $0.00100000=$ Minimum line-intensity \\ $0.00100000=$ Diagonalization criterium (not in use) \\ $800.20420000=\mathrm{FIELD}(1 \mathrm{H}, \mathrm{MHz})$, used to transform shifts to ppms \\ $12.25068342=$ Left frequency $(\mathrm{ppm})$ \\ $-1.69659414=$ Right frequency $(\mathrm{ppm})$ \\ $0.000=$ Acquisition time (s, for QMTLS) \\ $1.066=$ Line-width (for modes D, P \& T, 0=use defaults) \\ $0.057100577=$ Data-point resolution $(\mathrm{Hz})$ \\ $-2.128=$ GAUSSIAN ( $\%, 0=$ use default from INF) \\ $5.723=$ Dispersion contribution (\%, $0=$ use default from INF) \\ $0.00000000=$ Decoupling frequency (for DORES)
}

\title{
PMS file for 4
}

NMR-data: c:luserslvmuserldesktoplrasika_dentalprojectlpab3_perchlperch

\#\$ Date 10.10.2016; Time 15: 5:18 perch.pms

\section{ACTIVE SPECIES:1H}

CHEMICAL SHIFTS(PPM):

PROTON $2 *$ SPIN $=1$ SPECIES $=1 \mathrm{H} \quad$ POPULATION $(Y)=1.00000$

$\mathrm{H} 2 / 15.7874061 * 1 * 1$ STAT=Y PRED $=6.137$ RANGE= 0.150 WIDTH(Y)=1.177 RESP(Y)=0.6491 HSQC $=\mathrm{C} 2$

$\mathrm{H} 6 / 15.8873751 * 1 * 1$ STAT $=\mathrm{Y}$ PRED $=6.288 \mathrm{RANGE}=0.150 \mathrm{WIDTH}(\mathrm{Y})=1.306 \mathrm{RESP}(\mathrm{Y})=0.6759 \mathrm{HSQC}=\mathrm{C} 6$

$\mathrm{H} 7 / 14.4105891 * 1 * 1$ STAT=Y PRED $=4.688$ RANGE= $0.490 \mathrm{WIDTH}(\mathrm{Y})=1.361 \mathrm{RESP}(\mathrm{Y})=0.7089 \mathrm{HSQC}=\mathrm{C} 7$

$\mathrm{H} 8 / 14.3531081 * 1 * 1$ STAT $=\mathrm{Y}$ PRED $=4.452$ RANGE $=0.447 \mathrm{WIDTH}(\mathrm{Y})=1.531 \mathrm{RESP}(\mathrm{Y})=1.0000 \mathrm{HSQC}=\mathrm{C} 8$

$\mathrm{H} 9 / 14.2556291 * 1 * 1$ STAT=Y PRED $=4.793$ RANGE=0.380 WIDTH(Y)=1.354 RESP(Y)=0.6895 HSQC $=\mathrm{C} 9$

$\mathrm{H} 14 / 16.0716671 * 1 * 1$ STAT=Y PRED $=5.776$ RANGE $=0.737 \mathrm{WIDTH}(\mathrm{Y})=1.909 \operatorname{RESP}(\mathrm{Y})=0.6790 \mathrm{HSQC}=\mathrm{C} 14$

$\mathrm{H} 18 \mathrm{~A} / 12.4865401 * 1 * 1$ STAT=Y PRED $=2.691 \mathrm{RANGE}=0.202 \mathrm{WIDTH}(\mathrm{Y})=2.244 \operatorname{RESP}(\mathrm{Y})=0.7312 \mathrm{HSQC}=\mathrm{C} 18$

$\mathrm{H} 18 \mathrm{~B} / 12.7608291 * 1 * 1$ STAT $=\mathrm{Y}$ PRED $=2.835 \mathrm{RANGE}=0.277 \mathrm{WIDTH}(\mathrm{Y})=2.043 \mathrm{RESP}(\mathrm{Y})=0.7092 \mathrm{HSQC}=\mathrm{C} 18$

$\mathrm{H} 19 / 13.7918411 * 1 * 1$ STAT=Y PRED $=4.218$ RANGE $=0.235 \mathrm{WIDTH}(\mathrm{Y})=1.737 \mathrm{RESP}(\mathrm{Y})=0.6607 \mathrm{HSQC}=\mathrm{C} 19$

$\mathrm{H} 20 / 14.5405181 * 1 * 1$ STAT $=\mathrm{Y}$ PRED $=4.841$ RANGE $=0.370 \mathrm{WIDTH}(\mathrm{Y})=1.793 \mathrm{RESP}(\mathrm{Y})=0.7517 \mathrm{HSQC}=\mathrm{C} 20$

$\mathrm{H} 26 / 16.4663031 * 1 * 1$ STAT=Y PRED $=6.430$ RANGE $=0.770 \mathrm{WIDTH}(\mathrm{Y})=1.181 \mathrm{RESP}(\mathrm{Y})=0.6494 \mathrm{HSQC}=\mathrm{C} 26$

$\mathrm{H} 27 / 16.6718131 * 1 * 1$ STAT $=Y$ PRED $=6.576$ RANGE $=0.410 \mathrm{WIDTH}(\mathrm{Y})=1.304 \mathrm{RESP}(\mathrm{Y})=0.7002 \mathrm{HSQC}=\mathrm{C} 27$

$\mathrm{H} 30 / 16.7365161 * 1 * 1$ STAT $=Y$ PRED $=6.959$ RANGE $=0.307 \mathrm{WIDTH}(\mathrm{Y})=1.740 \mathrm{RESP}(\mathrm{Y})=0.8164 \mathrm{HSQC}=\mathrm{C} 30$

$\mathrm{H} 32 / 16.2522391 * 1 * 1$ STAT $=Y$ PRED $=6.865$ RANGE $=0.165$ WIDTH $(Y)=1.215$ RESP $(Y)=0.6319 \mathrm{HSQC}=\mathrm{C} 32$

$\mathrm{H} 33 / 16.6764721 * 1 * 1$ STAT $=Y$ PRED $=6.732$ RANGE $=0.212 \mathrm{WIDTH}(\mathrm{Y})=1.380 \mathrm{RESP}(\mathrm{Y})=0.7292 \mathrm{HSQC}=\mathrm{C} 33$

$\mathrm{H} 36 / 16.58912711^{*} 1$ 1 STAT=Y PRED= 6.722 RANGE=0.307 WIDTH(Y)=1.363 RESP $(\mathrm{Y})=0.6962 \mathrm{HSQC}=\mathrm{C} 36$ 
COUPLING CONSTANTS(HZ):

\begin{tabular}{|c|c|c|c|c|}
\hline J43_44 & 2.3699 & $\mathrm{~J} \mathrm{H} 2$ & $\mathrm{H} 6$ & $S T A T=Y \quad P R E D=2.184$ RANGE $=1.000$ \\
\hline J45_46 & 875 & $\mathrm{~J} \mathrm{H7}$ & $\mathrm{H} 8$ & STAT $=Y$ PRED $=2.812$ RANGE $=2.040$ \\
\hline 47 & 0.0266 & $\mathrm{~J} \mathrm{H} 7$ & $\mathrm{H} 9$ & STAT $=Y \quad P R E D=1.647$ RANGE $=1.080$ \\
\hline $6 \_47$ & 9.8379 & $\mathrm{~J} \mathrm{H} 8$ & $\mathrm{H} 9$ & STAT $=Y \quad P R E D=2.556$ RANGE $=2.000$ \\
\hline 57 & 32 & $\mathrm{~J} \mathrm{H9}$ & $\mathrm{H} 26$ & $S T A T=Y$ PRED $=-C$ \\
\hline 59 & 23 & & $\mathrm{H} 30$ & STAT $=Y$ PRED \\
\hline 50 & -16 & & H18 & \\
\hline & 771 & & -119 & STAT $=$ \\
\hline 52 & 0.1127 & & $3 \mathrm{~A} \mathrm{H} 20$ & STAT $=Y \quad P R E D=2.470$ RANGE $=1.000$ \\
\hline $0 \_51$ & 5.5307 & & 3B H19 & STAT $=Y \quad P R E D=4.530$ RANGE $=2.000$ \\
\hline 52 & 7.3628 & & $\mathrm{H} 2 \mathrm{O}$ & STAT $=Y \quad P R E D=1.431 \mathrm{RA}$ \\
\hline & -0 . & & $\mathrm{H} 32$ & STAT $=Y$ PRED $=$ \\
\hline D & & & $\mathrm{H} 36$ & STAT $=Y$ PRED \\
\hline & & & $\mathrm{H} 27$ & STAT $=Y \quad P R E D=\varepsilon$ \\
\hline 59 & 2.0371 & & $\mathrm{H} 30$ & $\mathrm{RED}=2.083$ \\
\hline 58_59 & 0.0245 & $\mathrm{~J} \mathrm{H} 27$ & $\mathrm{H} 30$ & STAT $=Y \quad P R E D=0.500$ RANGE $=1.000$ \\
\hline & 8.1 & & & RED $=8.185$ RANGE $=1.000$ \\
\hline & 2.0 & & & RED $=2.083$ RANGE $=1.000$ \\
\hline & & & & $=1.000$ \\
\hline
\end{tabular}

CONTROL PARAMETERS:

Solvent $=$ none $($ def. $99 \%$ enriched $)$

$1.000=$ Concentration $($ vol $\%$, def $=1.0 \%)$

$0.00100000=$ Minimum line-intensity

$0.00100000=$ Diagonalization criterium (not in use)

$600.13020000=$ FIELD $(1 \mathrm{H}, \mathrm{MHz})$, used to transform shifts to ppms

$12.27150983=$ Left frequency $(\mathrm{ppm})$

$-1.70745485=$ Right frequency $(\mathrm{ppm})$

$0.000=$ Acquisition time $(\mathrm{s}$, for QMTLS)

1.177 = Line-width (for modes D, P \& T, 0=use defaults)

$0.058852578=$ Data-point resolution $(\mathrm{Hz})$

$-10.744=$ GAUSSIAN (\%, $0=$ use default from INF)

$1.335=$ Dispersion contribution (\%, $0=$ use default from INF)

$0.00000000=$ Decoupling frequency (for DORES)

\section{PMS file for 5}

NMR-data: c:lusers|vmuserldesktoplrasika_dentalproject|ngd05।perch

\#\$ Date 19.10.2016; Time 10:23:34 perch.pms

ACTIVE SPECIES:1H

CHEMICAL SHIFTS(PPM):

PROTON $2 *$ SPIN $=1$ SPECIES $=1 \mathrm{H} \quad$ POPULATION $(\mathrm{Y})=1.00000$

$\mathrm{H} 2 / 15.8975271 * 1 * 1$ STAT=Y PRED $=5.837 \mathrm{RANGE}=0.360 \mathrm{WIDTH}(\mathrm{Y})=2.641 \operatorname{RESP}(\mathrm{Y})=0.7291 \mathrm{HSQC}=\mathrm{C} 2$

$\mathrm{H} 6 / 15.9683801 * 1 * 1$ STAT $=Y$ PRED $=5.907$ RANGE $=0.200 \mathrm{WIDTH}(\mathrm{Y})=1.692 \mathrm{RESP}(\mathrm{Y})=0.5995 \mathrm{HSQC}=\mathrm{C} 6$

$\mathrm{H} 7 / 14.6276651 * 1 * 1$ STAT=Y PRED $=4.624$ RANGE $=0.504 \operatorname{WIDTH}(\mathrm{Y})=3.008 \mathrm{RESP}(\mathrm{Y})=0.7480 \mathrm{HSQC}=\mathrm{C} 7$

$\mathrm{H} 8 / 14.2298551 * 1 * 1$ STAT=Y PRED $=4.096$ RANGE=0.440 WIDTH $(\mathrm{Y})=3.080 \mathrm{RESP}(\mathrm{Y})=0.7514 \mathrm{HSQC}=\mathrm{C} 8$

$\mathrm{H} 9 / 15.0842721 * 1 * 1$ STAT=Y PRED $=5.317$ RANGE= $0.325 \mathrm{WIDTH}(\mathrm{Y})=3.100 \mathrm{RESP}(\mathrm{Y})=0.8726 \mathrm{HSQC}=\mathrm{C} 9$

$\mathrm{H} 13 / 16.763741 \quad 1 * 1 * 1$ STAT $=\mathrm{Y}$ PRED $=6.625 \mathrm{RANGE}=0.150 \mathrm{WIDTH}(\mathrm{Y})=1.833 \mathrm{RESP}(\mathrm{Y})=0.8074 \mathrm{HSQC}=\mathrm{C} 13$

$\mathrm{H} 14 / 16.74134611^{*}{ }^{*} 1$ STAT=Y PRED= 6.747 RANGE=0.199 WIDTH $(\mathrm{Y})=1.800 \mathrm{RESP}(\mathrm{Y})=0.8074 \mathrm{HSQC}=\mathrm{C} 14$

$\mathrm{H} 17 / 16.9579041 * 1 * 1$ STAT $=Y$ PRED $=7.188$ RANGE $=0.150 \mathrm{WIDTH}(\mathrm{Y})=1.823 \mathrm{RESP}(\mathrm{Y})=0.8319 \mathrm{HSQC}=\mathrm{C} 17$

$\mathrm{H} 22 / 15.8488061 * 1 * 1$ STAT=Y PRED $=5.970$ RANGE $=0.500 \mathrm{WIDTH}(\mathrm{Y})=2.842 \mathrm{RESP}(\mathrm{Y})=0.7583 \mathrm{HSQC}=\mathrm{C} 22$

$\mathrm{H} 26 \mathrm{~A} / 12.9597091 * 1 * 1$ STAT $=\mathrm{Y}$ PRED $=2.726 \mathrm{RANGE}=0.239 \mathrm{WIDTH}(\mathrm{Y})=4.098 \mathrm{RESP}(\mathrm{Y})=0.8349 \mathrm{HSQC}=\mathrm{C} 26$

$\mathrm{H} 26 \mathrm{~B} / 12.84702611^{*} 1 * 1$ STAT=Y PRED $=2.304 \mathrm{RANGE}=0.340 \mathrm{WIDTH}(\mathrm{Y})=5.288 \mathrm{RESP}(\mathrm{Y})=1.0000 \mathrm{HSQC}=\mathrm{C} 26$

$\mathrm{H} 27 / 14.2324171 * 1 * 1$ STAT $=\mathrm{Y}$ PRED $=3.906$ RANGE $=0.394 \operatorname{WIDTH}(\mathrm{Y})=3.064 \mathrm{RESP}(\mathrm{Y})=0.8084 \mathrm{HSQC}=\mathrm{C} 27$

$\mathrm{H} 28 / 15.0315131 * 1 * 1$ STAT $=\mathrm{Y}$ PRED $=4.756$ RANGE $=0.380 \mathrm{WIDTH}(\mathrm{Y})=3.436 \mathrm{RESP}(\mathrm{Y})=0.8817 \mathrm{HSQC}=\mathrm{C} 28$

$\mathrm{H} 32 / 16.7495381 * 1 * 1$ STAT $=\mathrm{Y}$ PRED $=6.651 \mathrm{RANGE}=0.265 \mathrm{WIDTH}(\mathrm{Y})=2.833 \mathrm{RESP}(\mathrm{Y})=0.8074 \mathrm{HSQC}=\mathrm{C} 32$

$\mathrm{H} 33 / 16.7488761 * 1 * 1$ STAT $=\mathrm{Y}$ PRED $=6.725 \mathrm{RANGE}=0.245 \mathrm{WIDTH}(\mathrm{Y})=2.833 \mathrm{RESP}(\mathrm{Y})=0.8074 \mathrm{HSQC}=\mathrm{C} 33$

$\mathrm{H} 36 / 17.0578071 * 1 * 1$ STAT $=Y$ PRED $=6.762$ RANGE $=0.710$ WIDTH $(Y)=2.438$ RESP $(Y)=0.7582 \mathrm{HSQC}=\mathrm{C} 36$

COUPLING CONSTANTS(HZ):

J43 $44 \quad 2.2312 \quad \mathrm{~J} \mathrm{H} 2 \quad \mathrm{H} 6 \quad \mathrm{STAT}=\mathrm{Y}$ PRED $=2.184$ RANGE $=1.000$

J45_46 1.9208 J H7 H8 STAT=Y PRED $=2.812$ RANGE $=2.040$ 


\begin{tabular}{|c|c|c|c|}
\hline J46_47 & 0.6865 & $\mathrm{JH} 8 \mathrm{H} 9$ & STAT $=Y \quad P R E D=2.576$ RANGE $=2.040$ \\
\hline J49_50 & 8.2885 & J H13 H14 & STAT $=Y \quad P R E D=8.186$ RANGE $=1.000$ \\
\hline J49-51 & 1.9868 & J H13 H17 & STAT $=Y \quad P R E D=2.083$ RANGE $=1.000$ \\
\hline J50_51 & 283 & $\mathrm{~J} \mathrm{H} 14 \quad \mathrm{H} 17$ & STAT $=Y \quad P R E D$ \\
\hline J55 56 & -16.5634 & $\mathrm{~J} \mathrm{H} 26 \mathrm{~A} \mathrm{H} 26 \mathrm{E}$ & $B$ STAT $=Y$ PRED \\
\hline J55 57 & 4.5570 & J H26A H27 & STAT $=Y \quad$ PRED $=4.393$ RANGE $=2.000$ \\
\hline J55_58 & 0.0049 & $\mathrm{~J} \mathrm{H} 26 \mathrm{~A} \mathrm{H} 28$ & STAT $=Y \quad P R E D=2.520$ RANGE $=1.000$ \\
\hline J56_57 & 2.5190 & J H26B H27 & STAT $=Y \quad P R E D=11.735$ RANGE $=2.000$ \\
\hline J57_58 & 0.7301 & $\mathrm{~J} \mathrm{H} 27$ H28 & STAT $=Y \quad P R E D=3.463$ RANGE $=2.000$ \\
\hline J60_61 & 11.5438 & $\mathrm{~J} \mathrm{H} 32 \mathrm{H} 33$ & STAT $=Y \quad P R E D=8.185$ RANGE $=1.000$ \\
\hline J60_62 & 1.6112 & J H32 H36 & $\mathrm{STAT}=\mathrm{Y} \quad \mathrm{PRED}=2.083 \mathrm{RANGE}=1.000$ \\
\hline J61_62 & 0.3518 & J H33 H36 & STAT $=Y \quad P R E D=0.500$ RANGE $=1.000$ \\
\hline
\end{tabular}

CONTROL PARAMETERS:

Solvent $=$ none $($ def. $99 \%$ enriched $)$

$1.000=$ Concentration $($ vol $\%$, def $=1.0 \%)$

$0.00100000=$ Minimum line-intensity

$0.00100000=$ Diagonalization criterium (not in use)

$800.20420000=$ FIELD $(1 \mathrm{H}, \mathrm{MHz})$, used to transform shifts to ppms

$12.25030144=$ Left frequency $(\mathrm{ppm})$

$-1.69697460=$ Right frequency $(\mathrm{ppm})$

$10.000=$ Acquisition time (s, for QMTLS)

$0.000=$ Line-width (for modes D, P \& T, 0=use defaults)

$0.084618981=$ Data-point resolution $(\mathrm{Hz})$

$10.325=$ GAUSSIAN (\%, 0=use default from INF)

$5.632=$ Dispersion contribution (\%, $0=$ use default from INF)

$0.00000000=$ Decoupling frequency $($ for DORES)

\section{PMS file for 6}

NMR-data: C:IUserslvmuser|Desktop|Rasika_dentalProjectINGD06INGD06LT/perch

\#\$ Date 8.10.2016; Time 15:28:16 perch.pms

ACTIVE SPECIES:1H

CHEMICAL SHIFTS(PPM):

Major $2 *$ SPIN $=1$ SPECIES $=1 \mathrm{H} \quad$ POPULATION $(\mathrm{Y})=0.66724$

$\mathrm{H} 1 / 15.6527011 * 1 * 1$ STAT $=Y$ PRED $=5.877$ RANGE $=0.150 \operatorname{WIDTH}(\mathrm{Y})=1.686 \operatorname{RESP}(\mathrm{Y})=1.0000 \mathrm{HSQC}=\mathrm{C} 1$

$\mathrm{H} 4 / 15.7127221 * 1 * 1$ STAT=Y PRED= 5.967 RANGE=0.175 WIDTH(Y)=1.485 RESP(Y)=1.0000 HSQC $=\mathrm{C} 4$

$\mathrm{H} 8 / 14.3850301 * 1 * 1$ STAT $=\mathrm{Y}$ PRED $=4.740 \mathrm{RANGE}=0.314 \mathrm{WIDTH}(\mathrm{Y})=1.499 \operatorname{RESP}(\mathrm{Y})=1.0000 \mathrm{HSQC}=\mathrm{C} 8$

$\mathrm{H} 9 / 14.6393001 * 1 * 1$ STAT=Y PRED $=4.115$ RANGE= $0.475 \mathrm{WIDTH}(\mathrm{Y})=1.629 \mathrm{RESP}(\mathrm{Y})=1.0000 \mathrm{HSQC}=\mathrm{C} 9$

$\mathrm{H} 10 / 14.4810081 * 1 * 1$ STAT $=\mathrm{Y}$ PRED $=4.446 \mathrm{RANGE}=0.500 \mathrm{WIDTH}(\mathrm{Y})=1.321 \mathrm{RESP}(\mathrm{Y})=1.0000 \mathrm{HSQC}=\mathrm{C} 10$

$\mathrm{H} 14 / 16.0683381 * 1 * 1$ STAT $=Y$ PRED $=5.891$ RANGE $=0.515 \mathrm{WIDTH}(\mathrm{Y})=3.063 \mathrm{RESP}(\mathrm{Y})=1.0000 \mathrm{HSQC}=\mathrm{C} 14$

$\mathrm{H} 18 / 16.9482661 * 1 * 1$ STAT $=\mathrm{Y}$ PRED $=6.771 \mathrm{RANGE}=0.225 \mathrm{WIDTH}(\mathrm{Y})=1.513 \mathrm{RESP}(\mathrm{Y})=1.0000 \mathrm{HSQC}=\mathrm{C} 18$

$\mathrm{H} 21 / 16.780567 \quad 1^{*} 1 * 1$ STAT $=\mathrm{Y}$ PRED $=6.631$ RANGE $=0.725 \mathrm{WIDTH}(\mathrm{Y})=1.722 \mathrm{RESP}(\mathrm{Y})=1.0000 \mathrm{HSQC}=\mathrm{C} 21$

$\mathrm{H} 22 / 16.7333521 * 1 * 1$ STAT $=\mathrm{Y}$ PRED $=6.620 \mathrm{RANGE}=0.275 \mathrm{WIDTH}(\mathrm{Y})=1.316 \mathrm{RESP}(\mathrm{Y})=1.0000 \mathrm{HSQC}=\mathrm{C} 22$

$\mathrm{H} 24 / 14.5368291 * 1 * 1$ STAT $=Y$ PRED $=4.791$ RANGE $=0.380 \mathrm{WIDTH}(\mathrm{Y})=2.224 \mathrm{RESP}(\mathrm{Y})=1.0000 \mathrm{HSQC}=\mathrm{C} 24$

$\mathrm{H} 25 / 14.1518391 * 1 * 1$ STAT $=Y$ PRED $=3.819$ RANGE $=0.385$ WIDTH(Y $)=2.303$ RESP $(Y)=1.0000 \mathrm{HSQC}=\mathrm{C} 25$

$\mathrm{H} 26 \mathrm{~A} / 12.6893801 * 1 * 1 \mathrm{STAT}=\mathrm{Y}$ PRED= 2.721 RANGE $=0.230 \mathrm{WIDTH}(\mathrm{Y})=3.325 \mathrm{RESP}(\mathrm{Y})=1.0000 \mathrm{HSQC}=\mathrm{C} 26$

$\mathrm{H} 26 \mathrm{~B} / 12.8372641 * 1 * 1$ STAT $=\mathrm{Y}$ PRED $=2.435 \mathrm{RANGE}=0.325 \mathrm{WIDTH}(\mathrm{Y})=2.949 \mathrm{RESP}(\mathrm{Y})=1.0000 \mathrm{HSQC}=\mathrm{C} 26$

$\mathrm{H} 28 / 16.7874531 * 1 * 1$ STAT $=\mathrm{Y}$ PRED $=6.787 \mathrm{RANGE}=0.570 \mathrm{WIDTH}(\mathrm{Y})=1.397 \mathrm{RESP}(\mathrm{Y})=1.0000 \mathrm{HSQC}=\mathrm{C} 28$

$\mathrm{H} 31 / 16.7765961 * 1 * 1$ STAT=Y PRED $=6.757$ RANGE $=0.235 \mathrm{WIDTH}(\mathrm{Y})=1.288 \mathrm{RESP}(\mathrm{Y})=1.0000 \mathrm{HSQC}=\mathrm{C} 31$

$\mathrm{H} 32 / 16.9010941 * 1 * 1$ STAT $=Y$ PRED $=6.637$ RANGE $=0.150 \mathrm{WIDTH}(\mathrm{Y})=1.659 \operatorname{RESP}(\mathrm{Y})=1.0000 \mathrm{HSQC}=\mathrm{C} 32$

Minor $2 *$ SPIN $=1$ SPECIES $=1 \mathrm{H} \quad$ POPULATION $(Y)=0.33276$

U1 / $25.82737611^{*} 1^{* 1}$ STAT $=Y$ PRED $=5.877$ RANGE $=0.150 \mathrm{WIDTH}(\mathrm{Y})=1.763 \operatorname{RESP}(\mathrm{Y})=1.0000 \mathrm{HSQC}=\mathrm{C} 1$

U4 / $25.8495451 * 1 * 1$ STAT=Y PRED $=5.967$ RANGE $=0.175 \mathrm{WIDTH}(\mathrm{Y})=1.387 \mathrm{RESP}(\mathrm{Y})=1.0000 \mathrm{HSQC}=\mathrm{C} 4$

U8 $/ 24.3287181 * 1 * 1$ STAT $=Y$ PRED $=4.740$ RANGE $=0.314 \operatorname{WIDTH}(\mathrm{Y})=1.524 \operatorname{RESP}(\mathrm{Y})=1.0000 \mathrm{HSQC}=\mathrm{C} 8$

U9 / $24.4960691 * 1 * 1$ STAT=Y PRED $=4.115$ RANGE= 0.475 WIDTH(Y)=1.719 RESP $(Y)=1.0000 \mathrm{HSQC}=\mathrm{C} 9$

$\mathrm{U} 10 / 24.5922751 * 1 * 1$ STAT $=\mathrm{Y}$ PRED $=4.446 \mathrm{RANGE}=0.500 \mathrm{WIDTH}(\mathrm{Y})=1.403 \mathrm{RESP}(\mathrm{Y})=1.0000 \mathrm{HSQC}=\mathrm{C} 10$

U14 / $25.9547411^{*} 1 * 1$ STAT=Y PRED $=5.891$ RANGE $=0.515 \mathrm{WIDTH}(\mathrm{Y})=3.003$ RESP $(\mathrm{Y})=1.0000 \mathrm{HSQC}=\mathrm{C} 14$

$\mathrm{U} 18 / 26.9014381 * 1 * 1$ STAT $=\mathrm{Y}$ PRED $=6.771 \mathrm{RANGE}=0.225 \mathrm{WIDTH}(\mathrm{Y})=1.585 \mathrm{RESP}(\mathrm{Y})=1.0000 \mathrm{HSQC}=\mathrm{C} 18$

$\mathrm{U} 21 / 26.7577951 * 1 * 1$ STAT=Y PRED $=6.631$ RANGE $=0.725 \mathrm{WIDTH}(\mathrm{Y})=1.780 \mathrm{RESP}(\mathrm{Y})=1.0000 \mathrm{HSQC}=\mathrm{C} 21$

$\mathrm{U} 22 / 26.7673351 * 1 * 1$ STAT $=\mathrm{Y}$ PRED=6.620 RANGE $=0.275 \mathrm{WIDTH}(\mathrm{Y})=1.571 \mathrm{RESP}(\mathrm{Y})=1.0000 \mathrm{HSQC}=\mathrm{C} 22$

U24 / $24.9722001 * 1 * 1$ STAT $=Y$ PRED $=4.791$ RANGE $=0.380 \mathrm{WIDTH}(\mathrm{Y})=2.555 \mathrm{RESP}(\mathrm{Y})=1.0000 \mathrm{HSQC}=\mathrm{C} 24$

$\mathrm{U} 25 / 24.1541351 * 1 * 1$ STAT $=Y$ PRED $=3.819$ RANGE $=0.385 \mathrm{WIDTH}(\mathrm{Y})=1.662 \mathrm{RESP}(\mathrm{Y})=1.0000 \mathrm{HSQC}=\mathrm{C} 25$

$\mathrm{U} 26 \mathrm{~A} / 22.8392891 * 1 * 1$ STAT $=\mathrm{Y}$ PRED $=2.721$ RANGE $=0.230 \mathrm{WIDTH}(\mathrm{Y})=2.880 \mathrm{RESP}(\mathrm{Y})=1.0000 \mathrm{HSQC}=\mathrm{C} 26$

$\mathrm{U} 26 \mathrm{~B} / 22.8927801{ }^{*} 1 * 1$ STAT=Y PRED $=2.435 \mathrm{RANGE}=0.325 \mathrm{WIDTH}(\mathrm{Y})=3.370 \mathrm{RESP}(\mathrm{Y})=1.0000 \mathrm{HSQC}=\mathrm{C} 26$ 
U28 / $27.0585901 * 1 * 1$ STAT $=Y$ PRED $=6.787$ RANGE $=0.570$ WIDTH $(\mathrm{Y})=1.396 \operatorname{RESP}(\mathrm{Y})=1.0000 \mathrm{HSQC}=\mathrm{C} 28$ U31 / $26.7197781 * 1 * 1$ STAT=Y PRED $=6.757$ RANGE= 0.235 WIDTH(Y)=1.465 RESP $(Y)=1.0000 \mathrm{HSQC}=\mathrm{C} 31$

$\mathrm{U} 32 / 26.8893621 * 1 * 1$ STAT=Y PRED $=6.637 \mathrm{RANGE}=0.150 \mathrm{WIDTH}(\mathrm{Y})=1.121 \operatorname{RESP}(\mathrm{Y})=1.0000 \mathrm{HSQC}=\mathrm{C} 32$

COUPLING CONSTANTS(HZ):

J53_54 2.2048 J H1 H4 STAT $=Y$ PRED $=2.184$ RANGE $=1.000$

J53_57 $0.0050 \quad \mathrm{~J} \mathrm{H} 1 \quad \mathrm{H} 10 \quad$ STAT $=Y$ PRED $=0.409$ RANGE $=1.000$

J54 57 $0.0059 \quad \mathrm{~J} \mathrm{H} 4 \quad \mathrm{H} 10 \quad \mathrm{STAT}=\mathrm{Y} \quad \mathrm{PRED}=0.415 \mathrm{RANGE}=1.000$

J55_56 9.8872 J H8 H9 STAT=Y PRED=9.761 RANGE $=2.800$

J55 $59 \quad-0.0001 \quad J$ H8 $\quad H 18 \quad S T A T=Y$ PRED $=-0.413$ RANGE $=1.000$

J55_60 $-0.5473 \quad \mathrm{~J} H 8$ H21 STAT $=Y$ PRED $=-0.587$ RANGE $=1.000$

J55 $61 \quad 0.0037 \quad \mathrm{~J} \mathrm{H} 8 \quad \mathrm{H} 22 \quad \mathrm{STAT}=\mathrm{Y} \quad \mathrm{PRED}=0.442 \mathrm{RANGE}=1.000$

J56_57 8.7755 J H9 H10 STAT=Y PRED $=11.873$ RANGE $=3.570$

J58 $64 \quad 0.0012 \quad \mathrm{~J} \mathrm{H} 14$ H26A STAT $=Y$ PRED $=0.433$ RANGE $=1.000$

J58_65 0.5775 J H14 H26B STAT $=Y$ PRED $=0.413$ RANGE $=1.000$

J59 $60 \quad 2.0001 \quad \mathrm{JH} 18$ H21 STAT=Y PRED $=2.083$ RANGE $=1.000$

J59_61 $0.0775 \mathrm{~J} \mathrm{H} 18$ H22 STAT=Y PRED $=0.500$ RANGE $=1.000$

J60_61 $8.0690 \quad \mathrm{~J} \mathrm{H} 21 \quad \mathrm{H} 22$ STAT $=Y$ PRED $=8.186$ RANGE $=1.000$

J62_63 1.4549 J H24 H25 STAT=Y PRED= 3.463 RANGE= 2.000

J62_64 $0.2871 \quad \mathrm{~J} \mathrm{H} 24$ H26A STAT $=Y$ PRED $=2.520$ RANGE $=1.000$

J62_66 $-0.5702 \quad \mathrm{~J}$ H24 H28 STAT $=Y$ PRED $=-0.614$ RANGE $=1.000$

J62_67 $0.0030 \quad \mathrm{~J} \mathrm{H} 24$ H31 STAT=Y PRED $=0.468$ RANGE $=1.000$

J62 $68 \quad-0.7874 \quad \mathrm{~J} \mathrm{H} 24$ H32 STAT $=Y$ PRED $=-0.597$ RANGE $=1.000$

J63_64 3.1742 J H25 H26A STAT=Y PRED $=3.986$ RANGE $=2.000$

J63_65 4.5496 J H25 H26B STAT $=Y$ PRED $=11.731$ RANGE $=2.000$

J64_65 $-16.7115 \mathrm{~J}$ H26A H26B STAT $=Y$ PRED $=-15.800$ RANGE $=1.340$

J66 $67 \quad 0.0204 \quad \mathrm{~J} \mathrm{H} 28$ H31 STAT $=Y$ PRED $=0.500$ RANGE $=1.000$

J66_68 2.0556 J H28 H32 STAT=Y PRED $=2.083$ RANGE $=1.000$

J67 $68 \quad 8.1544 \quad \mathrm{~J} \mathrm{H} 31 \mathrm{H} 32 \quad$ STAT $=Y$ PRED $=8.185$ RANGE $=1.000$

J153_54 2.2644 JU1 U4 STAT $=Y$ PRED $=2.184$ RANGE $=1.000$

$\mathrm{J} 15357 \quad 0.0004 \quad \mathrm{~J} U 1 \quad \mathrm{U} 10 \quad \mathrm{STAT}=\mathrm{Y}$ PRED $=0.409$ RANGE $=1.000$

J154_57 $0.0214 \quad \mathrm{JU} 4 \quad \mathrm{U} 10 \quad \mathrm{STAT}=\mathrm{Y}$ PRED $=0.415 \mathrm{RANGE}=1.000$

$\mathrm{J} 15556 \quad 9.8927$ J U8 U9 STAT $=Y$ PRED $=9.761$ RANGE $=2.800$

J155_59 -0.0181 J U8 U18 STAT $=Y$ PRED $=-0.413$ RANGE $=1.000$

$\mathrm{J} 15560 \quad-0.1696 \quad \mathrm{~J} \cup 8 \quad \mathrm{U} 21 \quad \mathrm{STAT}=\mathrm{Y} \quad \mathrm{PRED}=-0.587$ RANGE $=1.000$

J155_61 0.0104 J U8 U22 STAT $=Y$ PRED $=0.442$ RANGE $=1.000$

J156_57 8.2673 J U9 U10 STAT $=Y$ PRED $=11.873$ RANGE $=3.570$

J158_64 $-0.0116 \quad \mathrm{~J} U 14$ U26A STAT $=Y$ PRED $=0.433$ RANGE $=1.000$

$\mathrm{J} 158 \_650.0393 \quad \mathrm{~J} U 14 \mathrm{U} 26 \mathrm{~B} \quad \mathrm{STAT}=\mathrm{Y}$ PRED $=0.413 \mathrm{RANGE}=1.000$

J159_60 2.0274 J U18 U21 STAT=Y PRED $=2.083$ RANGE $=1.000$

J159_61 $0.0097 \quad \mathrm{~J} U 18$ U22 STAT $=Y$ PRED $=0.500$ RANGE $=1.000$

J160_61 $8.0950 \quad \mathrm{~J} U 21$ U22 $\quad$ STAT $=Y$ PRED $=8.186$ RANGE $=1.000$

J162_63 $1.1728 \quad \mathrm{~J} U 24$ U25 STAT $=Y$ PRED $=3.463$ RANGE $=2.000$

J162_64 $0.0041 \quad \mathrm{~J} U 24$ U26A STAT $=Y$ PRED $=2.520$ RANGE $=1.000$

J162_66 -0.0062 J U24 U28 STAT $=Y$ PRED $=-0.614$ RANGE $=1.000$

J162_67 $0.0740 \quad \mathrm{~J} U 24$ U31 STAT $=Y \quad$ PRED $=0.468$ RANGE $=1.000$

J162_68 $-0.2806 \quad \mathrm{~J}$ U24 U32 STAT $=Y$ PRED $=-0.597$ RANGE $=1.000$

J163_64 3.5579 J U25 U26A STAT $=Y$ PRED $=3.986$ RANGE $=2.000$

J163_65 4.2029 J U25 U26B STAT $=Y$ PRED $=11.731$ RANGE $=2.000$

$\mathrm{J} 16465-16.2591 \quad \mathrm{~J}$ U26A U26B STAT $=Y \quad P R E D=-15.800$ RANGE $=1.340$

J166_67 0.0334 J U28 U31 STAT=Y PRED $=0.500$ RANGE $=1.000$

$\mathrm{J} 166 \quad 68-2.0186 \quad \mathrm{~J} U 28$ U32 STAT $=Y \quad P R E D=2.083$ RANGE $=1.000$

J167_68 8.1171 J U31 U32 STAT=Y PRED= 8.185 RANGE= 1.000

CONTROL PARAMETERS:

Solvent $=$ none $($ def. $99 \%$ enriched $)$

$1.000=$ Concentration $($ vol $\%$, def $=1.0 \%)$

$0.00100000=$ Minimum line-intensity

$0.00100000=$ Diagonalization criterium (not in use)

$800.20420000=\operatorname{FIELD}(1 \mathrm{H}, \mathrm{MHz})$, used to transform shifts to ppms

$12.25048572=$ Left frequency $(\mathrm{ppm})$

$-1.69679245=$ Right frequency $(\mathrm{ppm})$

$10.000=$ Acquisition time (s, for QMTLS)

$0.000=$ Line-width (for modes D, P \& T, $0=$ use defaults)

$0.056318433=$ Data-point resolution $(\mathrm{Hz})$

$6.499=$ GAUSSIAN $(\%, 0=$ use default from INF)

$2.973=$ Dispersion contribution (\%, 0=use default from INF)

$0.00000000=$ Decoupling frequency (for DORES) 


\section{PMS file for 7}

NMR-data: c:lusersIvmuserldesktoplrasika_dentalprojectlpab2_021perch

\#\$ Date 9.10.2016; Time 20:25:50 perch.pms

\section{ACTIVE SPECIES:1H}

CHEMICAL SHIFTS(PPM):

PROTON $2 *$ SPIN $=1$ SPECIES $=1 \mathrm{H} \quad$ POPULATION $(\mathrm{Y})=1.00000$

$\mathrm{H} 31 / 14.9619361 * 1 * 1$ STAT $=\mathrm{Y}$ PRED $=4.535$ RANGE $=0.335 \mathrm{WIDTH}(\mathrm{Y})=3.153 \mathrm{RESP}(\mathrm{Y})=0.8905 \mathrm{HSQC}=\mathrm{C} 31$

$\mathrm{H} 32 / 14.2619001 * 1 * 1$ STAT=Y PRED=3.936 RANGE $=0.270 \mathrm{WIDTH}(\mathrm{Y})=2.224 \mathrm{RESP}(\mathrm{Y})=0.8598 \mathrm{HSQC}=\mathrm{C} 32$

$\mathrm{H} 8 / 15.0560601 * 1 * 1$ STAT $=Y$ PRED $=5.449$ RANGE $=0.355 \mathrm{WIDTH}(\mathrm{Y})=2.374 \mathrm{RESP}(\mathrm{Y})=0.9062 \mathrm{HSQC}=\mathrm{C} 8$

$\mathrm{H} 9 / 13.8198121 * 1 * 1$ STAT $=\mathrm{Y}$ PRED $=4.284 \mathrm{RANGE}=0.424 \mathrm{WIDTH}(\mathrm{Y})=1.746 \operatorname{RESP}(\mathrm{Y})=0.8356 \mathrm{HSQC}=\mathrm{C} 9$

$\mathrm{H} 33 \mathrm{~A} / 12.9427141 * 1 * 1$ STAT $=\mathrm{Y}$ PRED $=2.744 \mathrm{RANGE}=0.200 \mathrm{WIDTH}(\mathrm{Y})=3.337 \mathrm{RESP}(\mathrm{Y})=0.8426 \mathrm{HSQC}=\mathrm{C} 33$

$\mathrm{H} 33 \mathrm{~B} / 12.8068531 * 1 * 1$ STAT $=\mathrm{Y}$ PRED $=2.660 \mathrm{RANGE}=0.180 \mathrm{WIDTH}(\mathrm{Y})=5.441 \mathrm{RESP}(\mathrm{Y})=0.8424 \mathrm{HSQC}=\mathrm{C} 33$

$\mathrm{H} 25 / 15.8829991 * 1 * 1$ STAT $=Y$ PRED $=5.902$ RANGE $=0.534 \mathrm{WIDTH}(\mathrm{Y})=1.232 \mathrm{RESP}(\mathrm{Y})=0.8970 \mathrm{HSQC}=\mathrm{C} 25$

$\mathrm{H} 4 / 15.9421131 * 1 * 1$ STAT=Y PRED= 5.945 RANGE=0.254 WIDTH(Y)=1.024 RESP(Y)=0.8479 HSQC=C4

$\mathrm{H} 1 / 15.9668991 * 1 * 1$ STAT $=\mathrm{Y}$ PRED $=5.815 \mathrm{RANGE}=0.340 \mathrm{WIDTH}(\mathrm{Y})=1.104 \operatorname{RESP}(\mathrm{Y})=0.9090 \mathrm{HSQC}=\mathrm{C} 1$

$\mathrm{H} 10 / 14.6304791 * 1 * 1$ STAT=Y PRED $=4.543$ RANGE $=0.479 \mathrm{WIDTH}(\mathrm{Y})=2.263 \operatorname{RESP}(\mathrm{Y})=0.9138 \mathrm{HSQC}=\mathrm{C} 10$

$\mathrm{H} 14 / 16.6238871 * 1 * 1$ STAT=Y PRED $=6.506$ RANGE $=0.150 \mathrm{WIDTH}(\mathrm{Y})=2.077 \mathrm{RESP}(\mathrm{Y})=1.0000 \mathrm{HSQC}=\mathrm{C} 14$

$\mathrm{H} 15 / 16.6881951 * 1 * 1$ STAT=Y PRED $=6.716$ RANGE $=0.150 \mathrm{WIDTH}(\mathrm{Y})=1.186 \operatorname{RESP}(\mathrm{Y})=0.8429 \mathrm{HSQC}=\mathrm{C} 15$

$\mathrm{H} 17 / 16.8462991 * 1 * 1$ STAT=Y PRED $=7.152 \mathrm{RANGE}=0.150 \mathrm{WIDTH}(\mathrm{Y})=1.449 \mathrm{RESP}(\mathrm{Y})=0.8613 \mathrm{HSQC}=\mathrm{C} 17$

$\mathrm{H} 35 / 16.86535611^{*} 1 * 1$ STAT=Y PRED $=6.911$ RANGE $=0.245 \mathrm{WIDTH}(\mathrm{Y})=1.876 \mathrm{RESP}(\mathrm{Y})=0.9543 \mathrm{HSQC}=\mathrm{C} 35$

$\mathrm{H} 36 / 16.7329821 * 1 * 1$ STAT=Y PRED $=6.683$ RANGE $=0.300 \mathrm{WIDTH}(\mathrm{Y})=1.384 \mathrm{RESP}(\mathrm{Y})=0.8905 \mathrm{HSQC}=\mathrm{C} 36$

$\mathrm{H} 39 / 17.1088441 * 1 * 1$ STAT=Y PRED= 6.687 RANGE=0.265 WIDTH $(\mathrm{Y})=1.645$ RESP $(\mathrm{Y})=0.8457 \mathrm{HSQC}=\mathrm{C} 39$

COUPLING CONSTANTS(HZ):

J53_54 1.1561 J H31 H32 STAT=Y PRED= 1.427 RANGE $=2.000$

J54_57 4.5969 J H32 H33A STAT $=Y$ PRED $=4.436$ RANGE $=2.000$

J54_58 2.8687 J H32 H33B STAT=Y PRED $=2.919$ RANGE $=2.000$

J55 $56 \quad 0.8519 \quad \mathrm{~J} \mathrm{H} 8$ H9 STAT $=Y$ PRED $=2.714$ RANGE $=2.000$

J56_62 2.1330 J H9 H10 STAT=Y PRED $=2.812$ RANGE $=2.040$

J57_58 $-16.7139 \mathrm{~J}$ H33A H33B STAT=Y PRED $=-15.928$ RANGE $=1.280$

J60_61 2.3572 J H4 H1 STAT=Y PRED $=2.184$ RANGE $=1.000$

J63_64 8.1914 J H14 H15 STAT=Y PRED $=8.186$ RANGE $=1.000$

J63_65 2.0388 J H14 H17 STAT=Y PRED $=2.083$ RANGE $=1.000$

J64_65 $0.2316 \quad \mathrm{~J} \mathrm{H} 15 \quad \mathrm{H} 17 \quad \mathrm{STAT}=\mathrm{Y}$ PRED $=0.500$ RANGE $=1.000$

J66_67 8.2331 J H35 H36 STAT=Y PRED $=8.185$ RANGE $=1.000$

J66_68 2.0335 J H35 H39 STAT $=Y$ PRED $=2.083$ RANGE $=1.000$

J67_68 0.0877 J H36 H39 STAT=Y PRED $=0.500$ RANGE $=1.000$

CONTROL PARAMETERS:

Solvent $=$ none $($ def. $99 \%$ enriched $)$

$1.000=$ Concentration $($ vol $\%$, def $=1.0 \%)$

$0.00100000=$ Minimum line-intensity

$0.00100000=$ Diagonalization criterium (not in use)

$800.20500000=\mathrm{FIELD}(1 \mathrm{H}, \mathrm{MHz})$, used to transform shifts to ppms

$13.12561458=$ Left frequency $(\mathrm{ppm})$

$-0.82167183=$ Right frequency $(\mathrm{ppm})$

$10.000=$ Acquisition time (s, for QMTLS)

$0.975=$ Line-width (for modes D, P \& T, 0=use defaults)

$0.051214404=$ Data-point resolution $(\mathrm{Hz})$

$37.757=$ GAUSSIAN $(\%, 0=$ use default from INF)

$4.363=$ Dispersion contribution (\%, $0=$ use default from INF)

$0.00000000=$ Decoupling frequency (for DORES) 


\section{PMS file for 8}

NMR-data: C:IUserslvmuser|Desktop|Rasika_dentalProject|GSE_trimers|PERCHI65

\#\$ Date 18. 9.2016; Time 13:19:52 perch.pms

ACTIVE SPECIES:1H

CHEMICAL SHIFTS(PPM):

PROTON $2 *$ SPIN $=1$ SPECIES $=1 \mathrm{H} \quad$ POPULATION $(Y)=1.00000$

$\mathrm{H} 2 / 15.9999081 * 1 * 1$ STAT $=\mathrm{Y}$ PRED= 5.912 RANGE $=0.380 \mathrm{WIDTH}(\mathrm{Y})=1.249 \operatorname{RESP}(\mathrm{Y})=0.6856 \mathrm{HSQC}=\mathrm{C} 2$

$\mathrm{H} 6 / 15.9639121 * 1 * 1$ STAT $=Y$ PRED $=5.821$ RANGE $=0.469 \mathrm{WIDTH}(\mathrm{Y})=1.186 \mathrm{RESP}(\mathrm{Y})=0.6770 \mathrm{HSQC}=\mathrm{C} 6$

$\mathrm{H} 7 / 14.6849341 * 1 * 1$ STAT $=\mathrm{Y}$ PRED $=4.477$ RANGE= $0.510 \mathrm{WIDTH}(\mathrm{Y})=2.383 \mathrm{RESP}(\mathrm{Y})=0.8251 \mathrm{HSQC}=\mathrm{C} 7$

$\mathrm{H} 8 / 13.9905431 * 1 * 1$ STAT $=Y$ PRED $=4.066$ RANGE $=0.445 \mathrm{WIDTH}(\mathrm{Y})=2.208 \mathrm{RESP}(\mathrm{Y})=0.7027 \mathrm{HSQC}=\mathrm{C} 8$

$\mathrm{H} 9 / 15.0855261 * 1 * 1$ STAT=Y PRED $=5.488$ RANGE $=0.365 \mathrm{WIDTH}(\mathrm{Y})=1.994 \operatorname{RESP}(\mathrm{Y})=0.6728 \mathrm{HSQC}=\mathrm{C9}$

$\mathrm{H} 13 / 16.9078001 * 1 * 1$ STAT=Y PRED $=7.032$ RANGE $=0.165 \mathrm{WIDTH}(\mathrm{Y})=1.941 \mathrm{RESP}(\mathrm{Y})=0.7396 \mathrm{HSQC}=\mathrm{C} 13$

$\mathrm{H} 16 / 16.7384191 * 1 * 1$ STAT=Y PRED $=6.721$ RANGE $=0.195 \mathrm{WIDTH}(\mathrm{Y})=2.124 \mathrm{RESP}(\mathrm{Y})=0.9536 \mathrm{HSQC}=\mathrm{C} 16$

$\mathrm{H} 17 / 16.6921971 * 1 * 1$ STAT $=Y$ PRED $=6.622$ RANGE $=0.150$ WIDTH $(\mathrm{N})=2.578$ RESP $(\mathrm{Y})=0.7398 \mathrm{HSQC}=\mathrm{C} 17$

$\mathrm{H} 23 / 15.8813201 * 1 * 1$ STAT=Y PRED $=5.919$ RANGE $=0.615 \mathrm{WIDTH}(\mathrm{Y})=1.331 \mathrm{RESP}(\mathrm{Y})=0.7056 \mathrm{HSQC}=\mathrm{C} 23$

$\mathrm{H} 28 / 14.0805431 * 1 * 1$ STAT $=Y$ PRED $=3.783$ RANGE $=0.460$ WIDTH $(Y)=2.768$ RESP $(Y)=0.6711 \mathrm{HSQC}=\mathrm{C} 28$

$\mathrm{H} 29 / 15.3150881 * 1 * 1$ STAT $=Y$ PRED $=5.010$ RANGE $=0.405$ WIDTH $(\mathrm{Y})=2.034 \operatorname{RESP}(\mathrm{Y})=0.6489 \mathrm{HSQC}=\mathrm{C} 29$

$\mathrm{H} 33 / 17.0457211 * 1 * 1$ STAT $=Y$ PRED $=6.930$ RANGE $=0.440 \mathrm{WIDTH}(\mathrm{N})=2.309 \mathrm{RESP}(\mathrm{Y})=0.7582 \mathrm{HSQC}=\mathrm{C} 33$

$\mathrm{H} 36 / 16.6871021 * 1 * 1$ STAT=Y PRED $=6.778$ RANGE $=0.285 \mathrm{WIDTH}(\mathrm{Y})=2.213 \mathrm{RESP}(\mathrm{Y})=0.9637 \mathrm{HSQC}=\mathrm{C} 36$

$\mathrm{H} 37 / 16.7873971 * 1 * 1$ STAT $=Y$ PRED $=7.012$ RANGE $=0.385$ WIDTH $(Y)=2.724$ RESP $(Y)=0.8218$ HSQC $=\mathrm{C} 37$

$\mathrm{H} 42 / 14.7742651 * 1 * 1$ STAT=Y PRED $=4.586$ RANGE $=0.490 \mathrm{WIDTH}(\mathrm{Y})=2.490 \mathrm{RESP}(\mathrm{Y})=0.7065 \mathrm{HSQC}=\mathrm{C} 42$

$\mathrm{H} 44 / 15.8932681 * 1 * 1$ STAT=Y PRED $=5.835$ RANGE $=0.585 \mathrm{WIDTH}(\mathrm{Y})=1.239 \mathrm{RESP}(\mathrm{Y})=0.6729 \mathrm{HSQC}=\mathrm{C} 44$

$\mathrm{H} 49 / 14.2141051 * 1 * 1$ STAT=Y PRED $=3.906$ RANGE $=0.325 \mathrm{WIDTH}(\mathrm{Y})=2.913 \mathrm{RESP}(\mathrm{Y})=0.7085 \mathrm{HSQC}=\mathrm{C} 49$

$\mathrm{H} 50 / 15.0604091 * 1 * 1$ STAT=Y PRED $=4.580$ RANGE $=0.355 \mathrm{WIDTH}(\mathrm{Y})=4.338 \mathrm{RESP}(\mathrm{Y})=0.7212 \mathrm{HSQC}=\mathrm{C} 50$

$\mathrm{H} 54 / 16.848657 \quad 1 * 1 * 1$ STAT=Y PRED $=6.987$ RANGE $=0.255 \mathrm{WIDTH}(\mathrm{Y})=3.185 \mathrm{RESP}(\mathrm{Y})=0.9011 \mathrm{HSQC}=\mathrm{C} 54$

$\mathrm{H} 57 / 16.71690611^{*} 1$ 1 STAT=Y PRED $=6.792 \mathrm{RANGE}=0.195 \mathrm{WIDTH}(\mathrm{Y})=2.357 \mathrm{RESP}(\mathrm{Y})=1.0000 \mathrm{HSQC}=\mathrm{C} 57$

$\mathrm{H} 58 / 16.9234591 * 1 * 1$ STAT $=\mathrm{Y}$ PRED $=6.699 \mathrm{RANGE}=0.215 \mathrm{WIDTH}(\mathrm{N})=2.447 \mathrm{RESP}(\mathrm{Y})=0.7734 \mathrm{HSQC}=\mathrm{C} 58$

$\mathrm{H} 63 \mathrm{~A} / 12.5278271 * 1 * 1$ STAT $=\mathrm{Y}$ PRED $=2.470 \mathrm{RANGE}=0.215 \mathrm{WIDTH}(\mathrm{Y})=7.038 \mathrm{RESP}(\mathrm{Y})=0.8183 \mathrm{HSQC}=\mathrm{C} 63$

$\mathrm{H} 63 \mathrm{~B} / 12.6210401 * 1 * 1$ STAT=Y PRED $=2.786$ RANGE $=0.189 \mathrm{WIDTH}(\mathrm{Y})=7.367 \mathrm{RESP}(\mathrm{Y})=0.9157 \mathrm{HSQC}=\mathrm{C} 63$

COUPLING CONSTANTS(HZ):

J64_65 2.3546 J H2 H6 STAT=Y PRED $=2.184$ RANGE $=1.000$

J66_67 1.9544 J H7 H8 STAT $=Y$ PRED $=2.812$ RANGE $=1.020$

J67_68 1.0821 J H8 H9 STAT=Y PRED $=2.720$ RANGE $=1.000$

J70_71 $-0.0998 \quad \mathrm{~J} \mathrm{H} 13$ H16 STAT $=Y$ PRED $=0.500$ RANGE $=1.000$

J70_72 $1.8468 \quad \mathrm{JH} 13 \mathrm{H} 17 \quad$ STAT $=Y$ PRED $=2.083$ RANGE $=1.000$

J71_72 $8.1661 \quad \mathrm{~J} \mathrm{H} 16$ H17 $\quad$ STAT=Y PRED $=8.186$ RANGE $=1.000$

J78_79 $1.2104 \quad \mathrm{~J} \mathrm{H} 28 \quad \mathrm{H} 29 \quad$ STAT $=Y$ PRED $=2.719$ RANGE $=1.000$

J78_88 $1.9373 \mathrm{~J}$ H28 H42 STAT=Y PRED $=2.811$ RANGE $=1.020$

J81 $82 \quad-0.0211 \quad \mathrm{~J} \mathrm{H} 33$ H36 STAT $=Y$ PRED $=0.500$ RANGE $=1.000$

J81_83 1.7716 J H33 H37 STAT=Y PRED $=2.083$ RANGE $=1.000$

J82 $83 \quad 8.2653 \quad \mathrm{~J} \mathrm{H} 36$ H37 STAT $=Y$ PRED $=8.186$ RANGE $=1.000$

J90_91 4.6939 J H49 H50 STAT=Y PRED $=9.745$ RANGE $=1.000$

J90 $100 \quad 4.6365$ J H49 H63A STAT=Y PRED $=11.731$ RANGE $=1.000$

J90_101 5.3454 J H49 H63B STAT $=Y$ PRED $=4.132$ RANGE $=1.000$

J93 $94 \quad 0.1232 \quad \mathrm{~J} \mathrm{H} 54 \mathrm{H} 57 \quad \mathrm{STAT}=Y$ PRED $=0.500$ RANGE $=1.000$

J93_95 1.7876 J H54 H58 STAT $=Y$ PRED $=2.083$ RANGE $=1.000$

$\mathrm{J} 9495 \quad 8.1824$ J H57 H58 STAT $=Y$ PRED $=8.185$ RANGE $=1.000$

J100_101 -16.0502 J H63A H63B STAT=Y PRED $=-15.802$ RANGE $=1.340$

CONTROL PARAMETERS:

Solvent $=$ none $($ def. $99 \%$ enriched $)$

$1.000=$ Concentration $($ vol $\%$, def $=1.0 \%)$

$0.00100000=$ Minimum line-intensity

$0.00100000=$ Diagonalization criterium (not in use)

$800.20420000=\operatorname{FIELD}(1 \mathrm{H}, \mathrm{MHz})$, used to transform shifts to ppms

$12.25056016=$ Left frequency $(\mathrm{ppm})$

$-1.69676988=$ Right frequency $(\mathrm{ppm})$

$0.000=$ Acquisition time (s, for QMTLS)

$1.418=$ Line-width (for modes D, P \& T, 0=use defaults)

$0.170301584=$ Data-point resolution $(\mathrm{Hz})$

$-30.089=$ GAUSSIAN (\%, 0=use default from INF)

$7.537=$ Dispersion contribution (\%, 0=use default from INF)

$0.00000000=$ Decoupling frequency (for DORES) 


\section{PMS file for 9}

\section{ACTIVE SPECIES:1H}

CHEMICAL SHIFTS(PPM):

PROTON $2 *$ SPIN $=1$ SPECIES $=1 \mathrm{H} \quad$ POPULATION $(\mathrm{Y})=0.99999$

$\mathrm{H} 2 / 16.00551811^{*} 1 * 1$ STAT $=\mathrm{Y}$ PRED $=5.954$ RANGE $=0.260 \mathrm{WIDTH}(\mathrm{Y})=1.123 \operatorname{RESP}(\mathrm{Y})=0.3762 \mathrm{HSQC}=\mathrm{C} 2$

$\mathrm{H} 6 / 15.9786921 * 1 * 1$ STAT=Y PRED $=5.892 \mathrm{RANGE}=0.260 \mathrm{WIDTH}(\mathrm{Y})=1.273 \mathrm{RESP}(\mathrm{Y})=0.4201 \mathrm{HSQC}=\mathrm{C} 6$

$\mathrm{H} 7 / 14.6936401 * 1 * 1$ STAT=Y PRED $=4.731$ RANGE $=0.500 \mathrm{WIDTH}(\mathrm{Y})=2.383 \mathrm{RESP}(\mathrm{Y})=0.5085 \mathrm{HSQC}=\mathrm{C} 7$

$\mathrm{H} 8 / 13.951287 \quad 1^{*} 1^{* 1}$ STAT=Y PRED= 4.325 RANGE=0.460 WIDTH(Y)=2.021 RESP $(\mathrm{Y})=0.4476 \mathrm{HSQC}=\mathrm{C} 8$

$\mathrm{H} 9 / 15.0748901 * 1 * 1$ STAT=Y PRED $=4.968$ RANGE= $0.385 \mathrm{WIDTH}(\mathrm{Y})=1.688 \operatorname{RESP}(\mathrm{Y})=0.3881 \mathrm{HSQC}=\mathrm{C} 9$

$\mathrm{H} 13 / 16.89675311^{*} 1 * 1$ STAT=Y PRED $=7.132 \mathrm{RANGE}=0.615 \mathrm{WIDTH}(\mathrm{Y})=2.061 \mathrm{RESP}(\mathrm{Y})=0.5367 \mathrm{HSQC}=\mathrm{C} 13$

$\mathrm{H} 16 / 16.7354321 * 1 * 1$ STAT=Y PRED $=6.683$ RANGE $=0.275 \mathrm{WIDTH}(\mathrm{Y})=1.859 \mathrm{RESP}(\mathrm{Y})=0.6188 \mathrm{HSQC}=\mathrm{C} 16$

$\mathrm{H} 17 / 16.6805441 * 1 * 1$ STAT=Y PRED $=6.919$ RANGE $=0.150 \mathrm{WIDTH}(\mathrm{Y})=2.168 \mathrm{RESP}(\mathrm{Y})=0.5260 \mathrm{HSQC}=\mathrm{C} 17$

$\mathrm{H} 23 / 15.8609621 * 1 * 1$ STAT=Y PRED $=5.934$ RANGE $=0.565 \mathrm{WIDTH}(\mathrm{Y})=1.627 \mathrm{RESP}(\mathrm{Y})=0.5342 \mathrm{HSQC}=\mathrm{C} 23$

$\mathrm{H} 28 / 14.0418621 * 1 * 1$ STAT $=Y$ PRED $=3.765$ RANGE $=0.475$ WIDTH $(Y)=2.216$ RESP $(Y)=0.4182 \mathrm{HSQC}=\mathrm{C} 28$

$\mathrm{H} 29 / 15.2332241 * 1 * 1$ STAT $=\mathrm{Y}$ PRED $=4.770$ RANGE $=0.395 \mathrm{WIDTH}(\mathrm{Y})=1.958 \mathrm{RESP}(\mathrm{Y})=0.4351 \mathrm{HSQC}=\mathrm{C} 29$

$\mathrm{H} 33 / 17.0753111 * 1 * 1$ STAT=Y PRED $=6.690$ RANGE $=0.695 \mathrm{WIDTH}(\mathrm{N})=1.648$ RESP $(\mathrm{Y})=0.4150 \mathrm{HSQC}=\mathrm{C} 33$

$\mathrm{H} 36 / 16.73231511^{*} 1 * 1$ STAT=Y PRED $=6.803$ RANGE $=0.150 \mathrm{WIDTH}(\mathrm{Y})=2.470 \mathrm{RESP}(\mathrm{Y})=0.5279 \mathrm{HSQC}=\mathrm{C} 36$

$\mathrm{H} 37 / 16.7331051 * 1 * 1$ STAT $=Y$ PRED $=6.677$ RANGE $=0.240$ WIDTH(Y $)=2.591$ RESP $(Y)=0.5605$ HSQC $=\mathrm{C} 37$

$\mathrm{H} 42 / 14.56519211^{*}{ }^{*} 1$ STAT=Y PRED $=4.110$ RANGE $=0.514 \mathrm{WIDTH}(\mathrm{Y})=2.270 \mathrm{RESP}(\mathrm{Y})=0.4727 \mathrm{HSQC}=\mathrm{C} 42$

$\mathrm{H} 44 / 15.9198131 * 1 * 1$ STAT $=Y$ PRED $=5.833$ RANGE $=0.590$ WIDTH $(Y)=1.340$ RESP $(Y)=0.4476$ HSQC $=\mathrm{C} 44$

$\mathrm{H} 49 / 13.8529041 * 1 * 1$ STAT $=Y$ PRED $=3.897$ RANGE $=0.305$ WIDTH $(Y)=3.471$ RESP $(Y)=0.6589 \mathrm{HSQC}=\mathrm{C} 49$

$\mathrm{H} 50 / 14.8007391 * 1 * 1$ STAT $=\mathrm{Y}$ PRED $=4.616$ RANGE $=0.275 \mathrm{WIDTH}(\mathrm{N})=2.455 \mathrm{RESP}(\mathrm{Y})=1.0000 \mathrm{HSQC}=\mathrm{C} 50$

$\mathrm{H} 54 / 16.9114611 * 1 * 1$ STAT=Y PRED $=6.880 \mathrm{RANGE}=0.610 \mathrm{WIDTH}(\mathrm{Y})=3.242 \operatorname{RESP}(\mathrm{Y})=0.6734 \mathrm{HSQC}=\mathrm{C} 54$

$\mathrm{H} 57 / 16.7452411 * 1 * 1$ STAT=Y PRED $=6.730$ RANGE $=0.270 \mathrm{WIDTH}(\mathrm{Y})=3.009 \mathrm{RESP}(\mathrm{Y})=0.6038 \mathrm{HSQC}=\mathrm{C} 57$

$\mathrm{H} 58 / 16.7459521 * 1 * 1$ STAT=Y PRED $=6.706$ RANGE $=0.165 \mathrm{WIDTH}(\mathrm{Y})=2.501 \mathrm{RESP}(\mathrm{Y})=0.6013 \mathrm{HSQC}=\mathrm{C} 58$

$\mathrm{H} 63 \mathrm{~A} / 12.9247911 * 1 * 1$ STAT=Y PRED $=2.823 \mathrm{RANGE}=0.170 \mathrm{WIDTH}(\mathrm{Y})=4.081 \mathrm{RESP}(\mathrm{Y})=0.5624 \mathrm{HSQC}=\mathrm{C63}$

$\mathrm{H} 63 \mathrm{~B} / 12.6179731 * 1 * 1$ STAT=Y PRED $=2.496 \mathrm{RANGE}=0.175 \mathrm{WIDTH}(\mathrm{Y})=4.975 \mathrm{RESP}(\mathrm{Y})=0.8013 \mathrm{HSQC}=\mathrm{C} 63$

Unk $2 *$ SPIN $=1$ SPECIES $=1 \mathrm{H} \quad$ POPULATION $(\mathrm{Y})=0.00001$

U23 / $24.80105011^{*} 1$ STAT=Y PRED= 5.934 RANGE=0.565 WIDTH(Y)=3.563 RESP $(Y)=0.5342 \mathrm{HSQC}=\mathrm{C} 23$

\begin{tabular}{|c|c|c|c|c|}
\hline \multicolumn{5}{|c|}{ COUPLING CONSTANTS(HZ): fixed } \\
\hline J64_65 & 2.3337 & $\mathrm{~J} \mathrm{H} 2$ & H6 & STAT $=Y \quad P R E D=2.184$ RANGE $=1.000$ \\
\hline J66_67 & 2.0058 & $\mathrm{~J} \mathrm{H} 7$ & $\mathrm{H} 8$ & STAT $=Y$ PRED $=11.862$ RANGE $=3.260$ \\
\hline J67_68 & 1.0911 & & $\mathrm{H} 9$ & STAT $=Y \quad P R E D=2.7$ \\
\hline & 0.0 & & $\mathrm{H} 16$ & STAT $=Y$ PRED \\
\hline 72 & 2.0238 & & $\mathrm{H} 17$ & 00 \\
\hline 72 & & J H16 & $\mathrm{H} 17$ & $S T A T=Y \quad P F$ \\
\hline J78_79 & 1.1 & & $\mathrm{H} 29$ & STAT $=Y$ PRED $=$ \\
\hline J78_88 & 1.9 & & $\mathrm{H} 42$ & $S T A T=Y \quad P R E D=$ \\
\hline J81-82 & 0.1 & & H36 & STAT $=Y$ PRED $=$ \\
\hline & & & H37 & $S T A T=Y P$ \\
\hline 83 & 8.6 & & $\mathrm{H} 37$ & STAT $=Y P$ \\
\hline $\mathrm{J} 9091$ & 7.3391 & 49 & $\mathrm{H} 50$ & $\mathrm{STAT}=\mathrm{N}$ PRED $=9.7$ \\
\hline 100 & 5.6194 & 49 & $9 \mathrm{H} 63 \mathrm{~A}$ & A $\quad S T A T=Y \quad P R E D=4.059$ RANGE $=2.000$ \\
\hline J90_101 & 8.4757 & J H49 & $9 \mathrm{H} 63 \mathrm{~B}$ & $B \quad S T A T=Y \quad P R E D=11.730$ RANGE $=2.000$ \\
\hline J93_94 & 0.0005 & J H54 & H57 & STAT $=Y \quad P R E D=0.500$ RANGE $=1.000$ \\
\hline J93_95 & 1.8380 & J H54 & H58 & $S T A T=Y \quad P R E D=2.083$ RANGE $=1.000$ \\
\hline & 8.6848 & & & $S T A T=Y \quad P R E D=8.185$ RANGE $=1.000$ \\
\hline & & & & \\
\hline
\end{tabular}

CONTROL PARAMETERS:

Solvent $=$ none $($ def. $99 \%$ enriched $)$

$1.000=$ Concentration $($ vol $\%$, def $=1.0 \%)$

$0.00100000=$ Minimum line-intensity

$0.00100000=$ Diagonalization criterium (not in use)

$800.20000000=\operatorname{FIELD}(1 \mathrm{H}, \mathrm{MHz})$, used to transform shifts to ppms

$12.25087997=$ Left frequency $(\mathrm{ppm})$

$-1.69641669=$ Right frequency $(\mathrm{ppm})$

$10.000=$ Acquisition time (s, for QMTLS)

$0.000=$ Line-width (for modes D, P \& T, $0=$ use defaults)

$0.085149489=$ Data-point resolution $(\mathrm{Hz})$

$-4.607=$ GAUSSIAN (\%, 0=use default from INF)

$7.683=$ Dispersion contribution (\%, $0=$ use default from INF)

$0.00000000=$ Decoupling frequency (for DORES) 
${ }^{1} \mathrm{H}$ and ${ }^{13} \mathrm{C}$ NMR chemical shifts for dimers $\mathbf{1}, \mathbf{4}$ and 7

\begin{tabular}{|c|c|c|c|c|c|c|}
\hline \multirow{2}{*}{ position } & \multicolumn{6}{|c|}{$\delta[\mathrm{ppm}]$, mult. $(J[\mathrm{~Hz}])$} \\
\hline & $\mathbf{1}, \delta^{1} \mathrm{H}$ & $\mathbf{1}, \delta^{13} \mathrm{C}$ & $4, \delta^{1} \mathrm{H}$ & $4, \delta^{13} \mathrm{C}$ & 7, $\delta^{1} \mathrm{H}$ & $7, \delta^{13} \mathrm{C}$ \\
\hline U-2 & $\begin{array}{l}5.1000, \mathrm{~d}( \\
<0.1)\end{array}$ & 77.06 & $\begin{array}{l}4.2556, \text { ddd } \\
(9.83,-0.53,<0.1)\end{array}$ & 83.80 & $\begin{array}{l}5.0560, d \\
(0.85)\end{array}$ & 76.89 \\
\hline U-3 & $\begin{array}{l}3.9398, \mathrm{dd} \\
(1.51,<0.1)\end{array}$ & 72.90 & $\begin{array}{l}4.3531, \mathrm{dd} \\
(7.98,9.83)\end{array}$ & 73.74 & $\begin{array}{l}3.8198, \mathrm{dd} \\
(0.85,2.13)\end{array}$ & 73.46 \\
\hline U-4 & $\begin{array}{l}4.6635, \mathrm{~d} \\
(1.51)\end{array}$ & 36.83 & $\begin{array}{l}4.4105, \mathrm{dd} \\
(7.98,<0.1)\end{array}$ & 38.35 & $\begin{array}{l}4.6304, d \\
(2.13)\end{array}$ & 36.72 \\
\hline U-6 & $\begin{array}{l}5.9291, \mathrm{~d} \\
(2.05)\end{array}$ & 95.87 & $\begin{array}{l}5.7874, \mathrm{~d} \\
(2.36)\end{array}$ & 96.47 & $\begin{array}{l}5.9421, \mathrm{~d} \\
(2.35)\end{array}$ & 95.88 \\
\hline U-8 & $\begin{array}{l}5.9477, \mathrm{~d} \\
(2.05)\end{array}$ & 95.31 & $\begin{array}{l}5.8873, \mathrm{~d} \\
(2.36)\end{array}$ & 97.03 & $\begin{array}{l}5.9668, \mathrm{~d} \\
(2.35)\end{array}$ & 95.71 \\
\hline U-2' & $\begin{array}{l}6.8749, \mathrm{dd} \\
(2.11,<0.1)\end{array}$ & 115.00 & $\begin{array}{l}6.7365, \mathrm{dd} \\
(2.03,<0.1)\end{array}$ & 116.23 & $\begin{array}{l}6.8462, \mathrm{dd} \\
(2.03,0.23)\end{array}$ & 114.92 \\
\hline $\mathrm{U}-5$ & $\begin{array}{l}6.7006, \mathrm{dd} \\
(8.23,<0.1)\end{array}$ & 115.61 & $\begin{array}{l}6.6718, \mathrm{dd} \\
(8.12,<0.1)\end{array}$ & 115.88 & $\begin{array}{l}6.6881, \mathrm{dd} \\
(8.19 .0 .23)\end{array}$ & 115.75 \\
\hline U-6' & $\begin{array}{l}6.6847, \mathrm{dd} \\
(8.23,2.11)\end{array}$ & 119.10 & $\begin{array}{l}6.4663, \mathrm{dd} \\
(8.12,2.03)\end{array}$ & 120.33 & $\begin{array}{l}6.6238, \mathrm{dd} \\
(8.19,2.03)\end{array}$ & 119.02 \\
\hline $\mathrm{U}-1$ & & 132.65 & & 132.46 & & 132.41 \\
\hline U-5 & & $157.82^{b}$ & & $158.55^{\mathrm{c}}$ & & $157.76^{\mathrm{b}}$ \\
\hline U-7 & & $158.02^{\mathrm{b}}$ & & $157.01^{\mathrm{c}}$ & & $157.81^{\mathrm{a}}$ \\
\hline $\mathrm{U}-8 \mathrm{a}$ & & $158.70^{\mathrm{b}}$ & & $157.20^{\mathrm{c}}$ & & $157.86^{\mathrm{a}}$ \\
\hline U-4a & & 101.92 & & 107.09 & & 101.74 \\
\hline $\mathrm{T}-2$ & $\begin{array}{l}4.9548, d \\
(5.39)\end{array}$ & 81.68 & $\begin{array}{l}4.5405, \text { ddd } \\
(7.36,-0.61,-0.52)\end{array}$ & 82.27 & $\begin{array}{l}4.9619, \mathrm{~d} \\
(1.15)\end{array}$ & 79.39 \\
\hline $\mathrm{T}-3$ & $\begin{array}{l}4.1618, \text { ddd }(5.39 \\
5.59,5.45)\end{array}$ & 68.16 & $\begin{array}{l}3.7918, \text { ddd } \\
(8.07,5.53,7.36)\end{array}$ & 68.88 & $\begin{array}{l}4.2619, \mathrm{ddd} \\
(1.15,4.59,2.86)\end{array}$ & 66.89 \\
\hline $\mathrm{T}-4 \mathrm{a}$ & $\begin{array}{l}2.5937, \mathrm{dd} \\
(5.59,-15.59)\end{array}$ & & $\begin{array}{l}2.4865, \mathrm{dd} \\
(-16.29,8.07)\end{array}$ & & $\begin{array}{l}2.8068, \mathrm{dd} \\
(-16.71,4.59)\end{array}$ & \\
\hline $\mathrm{T}-4 \mathrm{~b}$ & $\begin{array}{l}2.6011, \mathrm{dd} \\
(5.45,-15.59)\end{array}$ & 26.6 & $\begin{array}{l}2.7608, \mathrm{dd} \\
(-16.29,5.53)\end{array}$ & 28.81 & $\begin{array}{l}2.9427, \mathrm{dd} \\
(-16.71,2.86)\end{array}$ & 29.84 \\
\hline $\mathrm{T}-6$ & $5.8384, \mathrm{~s}$ & 96.72 & $6.0716, \mathrm{~s}$ & 95.45 & $5.8829, \mathrm{~s}$ & 96.91 \\
\hline T-2' & $\begin{array}{l}6.8557, \mathrm{dd} \\
(2.17,0.70)\end{array}$ & 114.00 & $\begin{array}{l}6.5891, \mathrm{dd} \\
(2.04,-0.52)\end{array}$ & 115.29 & $\begin{array}{l}7.1088, \mathrm{dd} \\
(2.09,<0.1)\end{array}$ & 114.92 \\
\hline $\mathrm{T}-5$ & $\begin{array}{l}6.7155, \mathrm{dd} \\
(8.19,0.70)\end{array}$ & 115.94 & $\begin{array}{l}6.6764, \mathrm{dd} \\
(8.12,<0.1)\end{array}$ & 115.97 & $\begin{array}{l}6.7329, \mathrm{dd} \\
(8.23,<0.1)\end{array}$ & 115.69 \\
\hline T-6' & $\begin{array}{l}6.8828, \mathrm{dd} \\
(8.19,2.17)\end{array}$ & 119.26 & $\begin{array}{l}6.2522, \text { ddd } \\
(8.17,2.04,-0.61)\end{array}$ & 119.63 & $\begin{array}{l}6.8653, \mathrm{dd} \\
(8.23,2.03)\end{array}$ & 118.77 \\
\hline $\mathrm{T}-8$ & & 107.64 & & 108.13 & & 107.14 \\
\hline $\mathrm{T}-1$ & & 132.32 & & 131.60 & & 132.03 \\
\hline $\mathrm{T}-5$ & & 155.7 & & 155.61 & & 156.43 \\
\hline $\mathrm{T}-7$ & & 156.37 & & 155.68 & & 156.51 \\
\hline $\mathrm{T}-8 \mathrm{a}$ & & 153.71 & & 154.69 & & 154.41 \\
\hline $\mathrm{T}-4 \mathrm{a}$ & & 100.17 & & 101.78 & & 99.84 \\
\hline
\end{tabular}

The $\delta{ }^{1} \mathrm{H}$ NMR $\delta$ values were obtained via HiFSA of the experimental NMR spectra acquired at $298 \mathrm{~K}$ for 4 on a $600 \mathrm{MHz}$ and $255 \mathrm{~K}$ for 1 and 7 on an $800 \mathrm{MHz}$ instruments. The ${ }^{13} \mathrm{C}$ NMR $\delta$ values for 1, 4 and 7 were obtained on a $900 \mathrm{MHz}$ NMR at $255 \mathrm{~K}$. a-c Assignments are interchangeable; The $\delta^{1} \mathrm{H}$ and $\delta^{13} \mathrm{C}$ for the major rotamers of $\mathbf{1 , 4}$ and $\mathbf{7}$ are presented in the table; U: upper unit, T: terminal unit 
${ }^{1} \mathrm{H}$ and ${ }^{13} \mathrm{C}$ NMR chemical shifts for trimer 8

\begin{tabular}{|l|l|l|}
\hline & \multicolumn{2}{|c|}{11} \\
\hline Position & $\delta_{\text {H }}(\mathbf{p p m}),(\boldsymbol{J}$ in Hz) & $\delta_{\mathbf{C}}(\mathbf{p p m})$ \\
\hline U-2 & $5.0855, \mathrm{~d}(1.08)$ & 76.80 \\
\hline U-3 & $3.9905, \mathrm{~d}(1.08,1.95)$ & 73.48 \\
\hline U-4 & $4.6849, \mathrm{~d}(1.95)$ & 36.98 \\
\hline U-6 & $5.9999, \mathrm{~d}(2.35)$ & $96.97^{\mathrm{a}}$ \\
\hline U-8 & $5.9639, \mathrm{~d}(2.35)$ & $96.84^{\mathrm{a}}$ \\
\hline U-4a & & 101.62 \\
\hline U-2 & $6.9078, \mathrm{~d}(1.84)$ & 114.81 \\
\hline U-5 & $6.6921, \mathrm{~d}(8.16)$ & 115.97 \\
\hline U-6 & $6.7384, \mathrm{dd}(8.16,1.84)$ & 119.03 \\
\hline M-2 & $5.3150, \mathrm{~d}(1.21)$ & 76.91 \\
\hline M-3 & $4.0805, \mathrm{dd}(1.21,1.93)$ & 72.31 \\
\hline M-4 & $4.7742, \mathrm{~d}(1.93)$ & 37.12 \\
\hline M-6 & $5.8813, \mathrm{~s}$ & 95.95 \\
\hline M-8 & & 106.37 \\
\hline M-4a & & 102.46 \\
\hline M-2 & $7.0457, \mathrm{~d}(1.77)$ & 114.92 \\
\hline M-5 & $6.6871, \mathrm{~d}(8.26)$ & 115.77 \\
\hline M-6 & $6.7873, \mathrm{dd}(8.26,1.77)$ & 118.64 \\
\hline M-8a & & 154.85 \\
\hline T-2 & $5.0604, \mathrm{~d}(4.69)$ & 81.57 \\
\hline T-3 & $4.2141, \mathrm{ddd}(4.69,4.63,5.34)$ & 68.10 \\
\hline T-4a & $2.5278, \mathrm{dd}(-16.05,4.63)$ & 26.27 \\
\hline T-4b & $2.6210, \mathrm{dd}(-16.05,5.34)$ & \\
\hline T-6 & $5.8932, \mathrm{~s}$ & 96.07 \\
\hline T-8 & & 108.06 \\
\hline T-C4a & & 100.17 \\
\hline T-2 & $6.8486, \mathrm{dd}(1.78,0.12)$ & 113.91 \\
\hline T-5 & $6.7169, \mathrm{dd}(8.18,0.12)$ & 115.82 \\
\hline T-6 & $6.9234, \mathrm{dd}(8.18,1.78)$ & 119.15 \\
\hline T-5 & & 155.73 \\
\hline T-8a & & 153.70 \\
\hline
\end{tabular}

a - interchangeable assignments. F1 resolution was not enough to unequivocally assign the U5/7/8a and M-5/7 carbon chemical shifts. 


\section{Phloroglucinolysis followed by chiral chromatography}

Prior to phloroglucinolysis, to generate standards for ent-catechin (C) and ent-epicatechin (E), which are not available commercially, an epimerization reaction was performed. Aqueous solutions of (+)-catechin and (-)-epicatechin were bubbled with $\mathrm{N} 2$ and heated at $95{ }^{\circ} \mathrm{C}$ for 1 hour in screw cap vials to achieve epimerization. Nitrogen was bubbled in order to deplete the oxygen and thereby minimize oxidative side reactions. The epimerization was confirmed by obtaining comparative chromatographic profiles of epimerized mixtures against the reference standards catechin and epicatechin. Catechin and epicatechin are epimerized to ent-epicatechin and ent-catechin respectively.

Phloroglucinolysis was performed for the determination of absolute configuration of the terminal monomeric unit of the PAC dimers. The phloroglucinol reaction products were separated on a C18-semi Preparative HPLC column to specifically isolate the cleaved terminal unit of the dimer and get rid of the excess of unreacted phloroglucinol before performing chiral chromatography. The following figure shows the C18- semi-prep HPLC profiles of phloroglucinolysis reaction products of dimers 2, 3, 4, 5, 6 and, 7. The HPLC peak i.d. of the phloroglucinolysis reaction products for dimer 7 (PA B2) was confirmed using ${ }^{1} \mathrm{H}$ NMR which provided guidance for the isolation of cleaved terminal units of rest of the compounds.

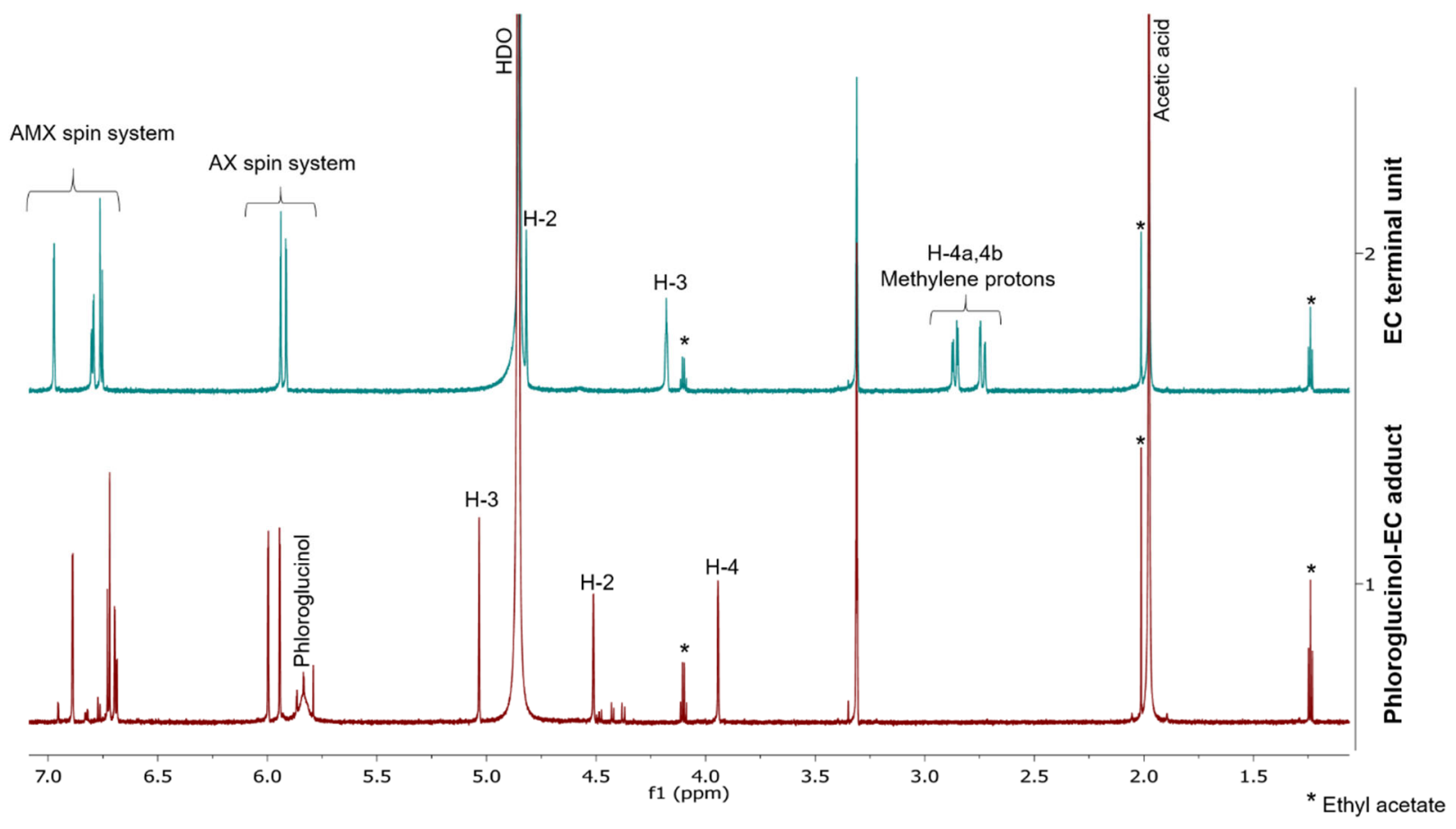



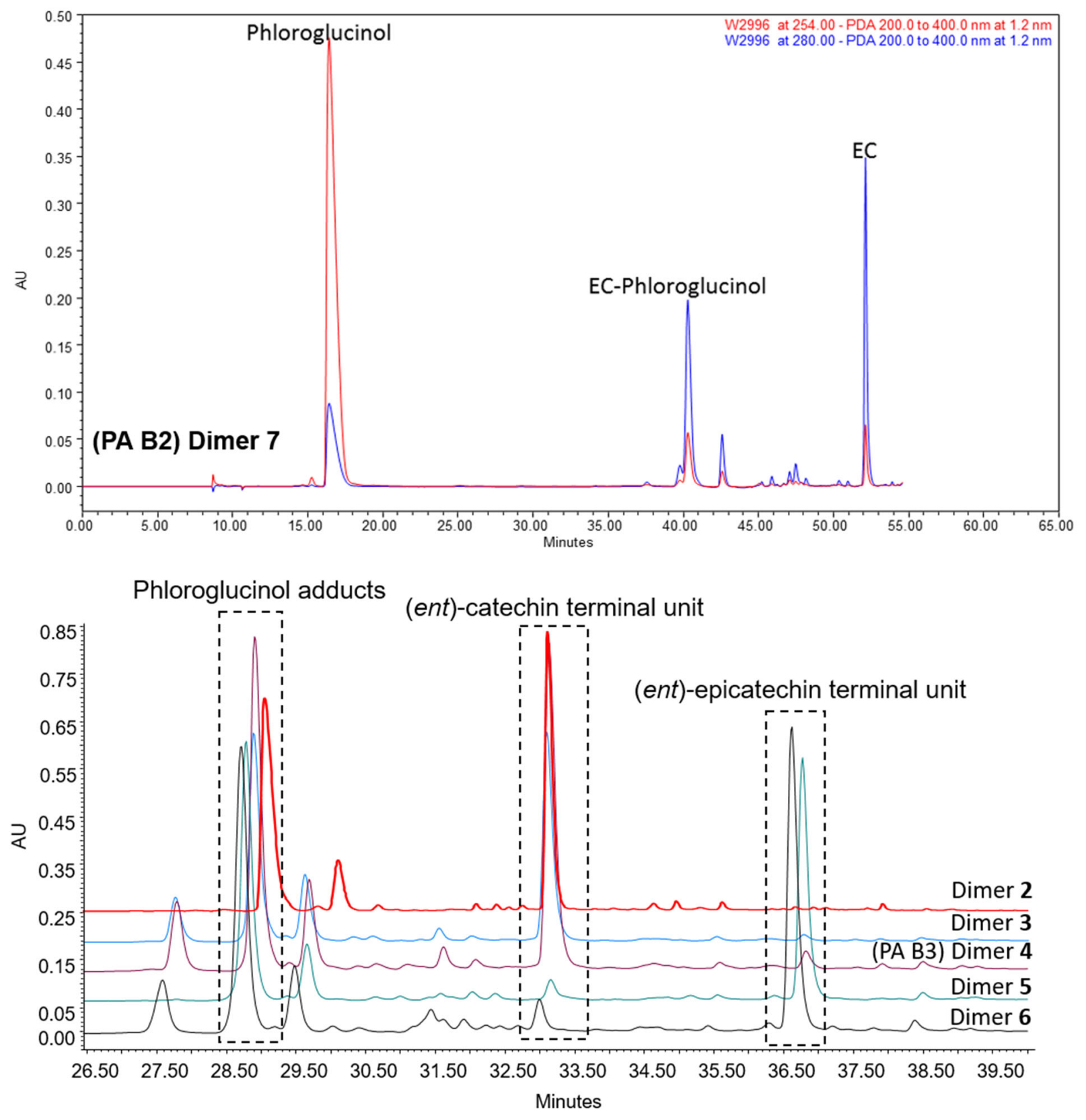

Each of the cleaved terminal units was analyzed using chiral chromatography to assign the absolute configuration based on comparison with the chiral profile of epimerized catechin and epicatechin. 
Dimer 2 has 2,3-cis and 2,3-trans configured upper and terminal units. Based on the chiral chromatogram, the terminal unit was confirmed to be (-)-catechin or ent-catechin.
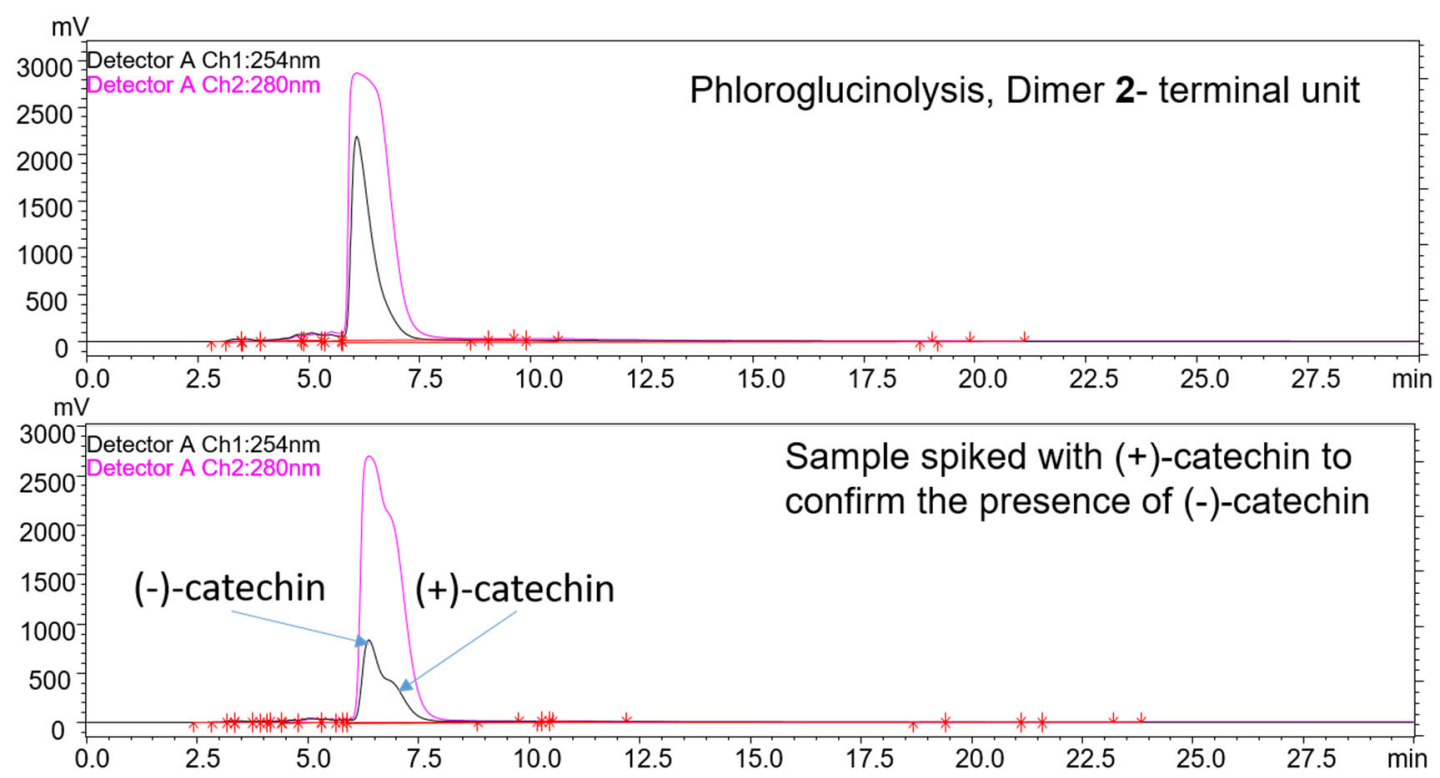

Dimer 5 has 2,3-cis configured upper and terminal units. Inversion of stereochemistry was also observed as seen by the presence of (-)-epicatechin peak, but $(+)$-epicatechin is the major product. Based on the chiral profile, the terminal unit was thus confirmed to be $(+)$-epicatechin or entepicatechin.

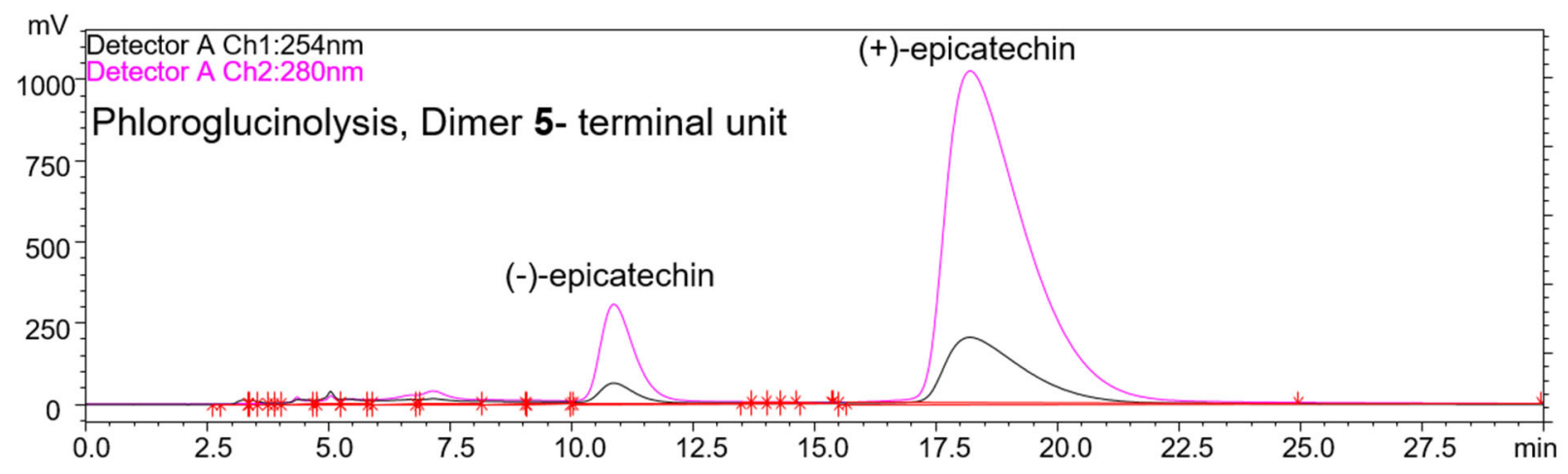


Dimer 6 has 2,3-trans and 2,3-cis configured upper and terminal units. Inversion of stereochemistry was also observed as seen by the presence of (+)-epicatechin peak, but (-)epicatechin is the major product. Based on the chiral profile, the terminal unit was thus confirmed to be (-)-epicatechin or ent-epicatechin.

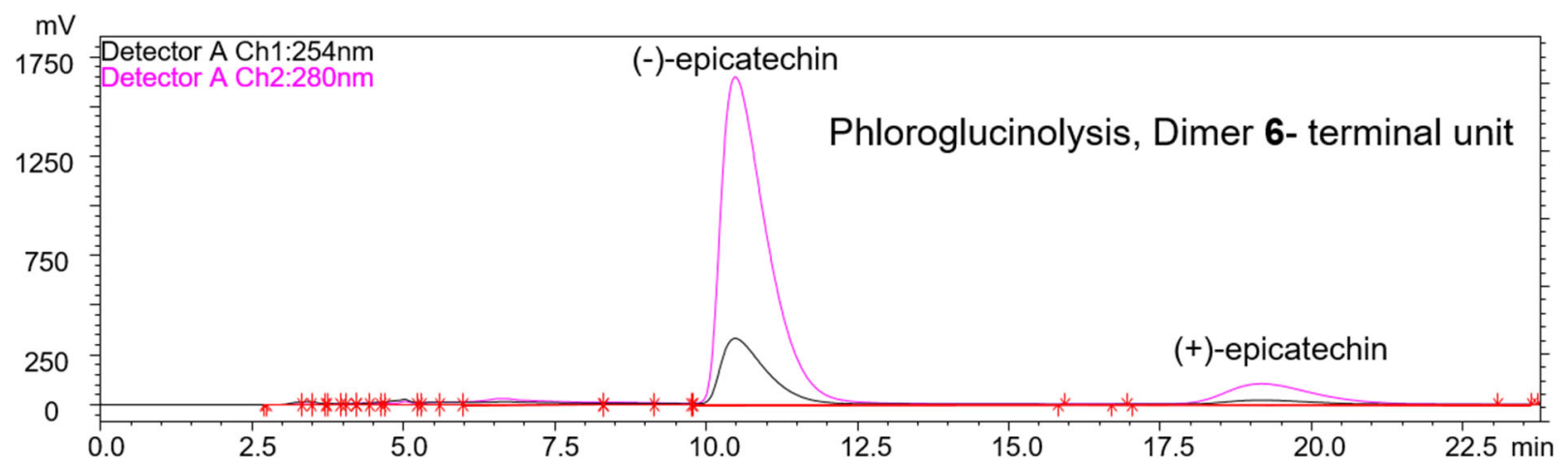

Dimer 3 has 2,3-trans configured upper and terminal units. Inversion of stereochemistry was also observed as seen by the presence of a small (-)-catechin peak, but (+)-catechin was the major product. Based on the chiral profile, the terminal unit was thus confirmed to be $(+)$-catechin.

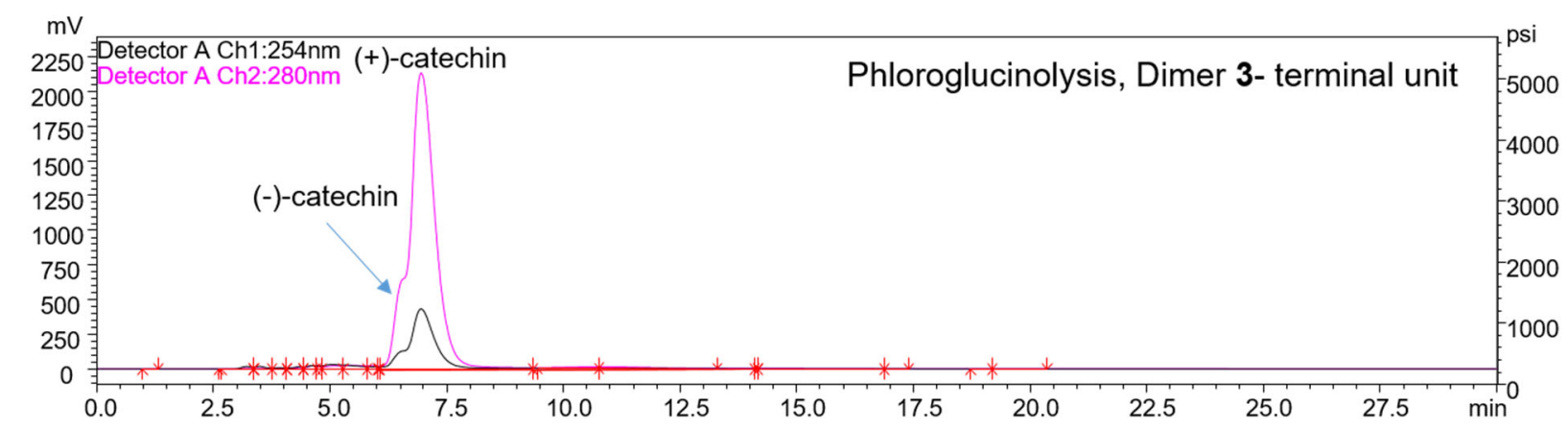


S9

DFT calculated ${ }^{13} \mathrm{C}$ NMR chemical shifts DP4+ analysis of compound 2

\begin{tabular}{|c|c|c|c|c|c|}
\hline Nuclei & Carbon i.d. & SP2? & $\begin{array}{c}\text { DP4+ } \\
\text { Experimental } \\
\end{array}$ & $\begin{array}{c}0.0 \% \\
\text { Isomer } 2 \mathrm{a}\end{array}$ & $\begin{array}{c}100 \% \\
\text { Isomer 2b }\end{array}$ \\
\hline C & U-2 & & 76.98 & 79.3 & 82.1 \\
\hline C & U-3 & & 73.59 & 76.0 & 78.0 \\
\hline C & U-4 & & 36.85 & 41.5 & 39.3 \\
\hline C & U-4a & $x$ & 101.43 & 110.9 & 104.0 \\
\hline C & U-1' & $x$ & 132.6 & 136.0 & 135.5 \\
\hline C & U-2' & $x$ & 114.95 & 127.8 & 123.3 \\
\hline C & U-6' & $x$ & 118.97 & 119.2 & 116.0 \\
\hline C & T-2 & & 82.71 & 86.1 & 86.4 \\
\hline C & T-3 & & 69.11 & 71.3 & 72.6 \\
\hline C & $\mathrm{T}-4$ & & 28.71 & 29.5 & 29.7 \\
\hline C & $\mathrm{T}-4 \mathrm{a}$ & $x$ & 100.7 & 104.4 & 104.5 \\
\hline C & T-5 & $x$ & 155.79 & 162.25 & 162.17 \\
\hline C & T-6 & $x$ & 96.79 & 97.58 & 100.13 \\
\hline C & $\mathrm{T}-7$ & $x$ & 156.54 & 161.35 & 165.69 \\
\hline C & T-8 & $x$ & 107.2 & 108.41 & 106.05 \\
\hline C & T-8a & $x$ & 154.07 & 163.08 & 160.71 \\
\hline C & T-1' & $x$ & 132.31 & 131.74 & 138.95 \\
\hline C & T-2' & $x$ & 114.09 & 118.92 & 118.92 \\
\hline$C$ & T-6' & $x$ & 120.05 & 126.95 & 127.88 \\
\hline
\end{tabular}

A, B and C-ring carbons with unequivocal chemical shift assignments were used for the DP4+ analysis https://sarotti-nmr.weebly.com/ of dimer 2. (Excel file is also included as a part of SI) 
DFT calculated ${ }^{13} \mathrm{C}$ NMR chemical shifts DP4+ analysis of compound $\mathbf{5}$

\begin{tabular}{|c|c|c|c|c|c|}
\hline Nuclei & Carbon i.d. & SP2? & $\begin{array}{c}\text { DP4+ } \\
\text { Experimental }\end{array}$ & $\begin{array}{c}100 \% \\
\text { Isomer 5a }\end{array}$ & $\begin{array}{c}0 \% \\
\text { Isomer 5b }\end{array}$ \\
\hline $\mathrm{C}$ & U-2 & & 77.21 & 79.7 & 81.7 \\
\hline $\mathrm{C}$ & U-3 & & 72.2 & 75.0 & 78.5 \\
\hline$C$ & U-4 & & 36.6 & 39.8 & 39.0 \\
\hline C & U-4a & $x$ & 101.49 & 108.5 & 102.5 \\
\hline$C$ & U-1' & $x$ & 132.64 & 133.0 & 134.5 \\
\hline C & U-2' & $x$ & 115.17 & 118.6 & 114.9 \\
\hline C & U-6' & $x$ & 119.29 & 125.8 & 121.1 \\
\hline C & $\mathrm{T}-2$ & & 79.32 & 84.9 & 84.8 \\
\hline C & T-3 & & 67.39 & 70.3 & 69.6 \\
\hline$C$ & $\mathrm{~T}-4$ & & 29.56 & 32.2 & 30.5 \\
\hline$C$ & $\mathrm{~T}-4 \mathrm{a}$ & $x$ & 99.78 & 103.1 & 98.6 \\
\hline$C$ & $\mathrm{~T}-5$ & $x$ & 156.16 & 163.33 & 163.14 \\
\hline$C$ & $\mathrm{~T}-6$ & $x$ & 96.77 & 98.62 & 100.07 \\
\hline$C$ & $\mathrm{~T}-7$ & $x$ & 156.28 & 162.69 & 167.06 \\
\hline$C$ & T-8 & $x$ & 107.82 & 106.41 & 106.43 \\
\hline C & T-8a & $x$ & 154.41 & 163.26 & 160.26 \\
\hline $\mathrm{C}$ & T-1' & $x$ & 132.73 & 133.01 & 139.74 \\
\hline C & T-2' & $x$ & 114.53 & 119.88 & 121.85 \\
\hline C & T-6' & $x$ & 118.3 & 124.17 & 125.47 \\
\hline
\end{tabular}

A, B and C-ring carbons with unequivocal chemical shift assignments were used for the DP4+ analysis https://sarotti-nmr.weebly.com/ of dimer 5. (Excel file is also included as a part of SI) 


\section{S9 Dentin Stiffness Assessment}

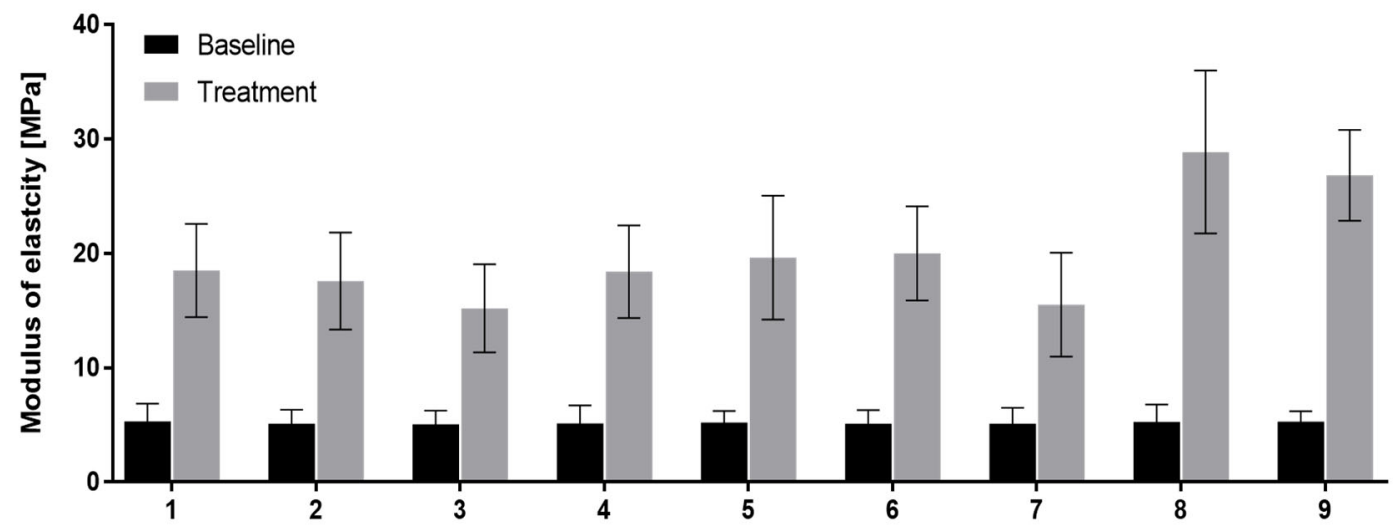

S10 Collagenase assay
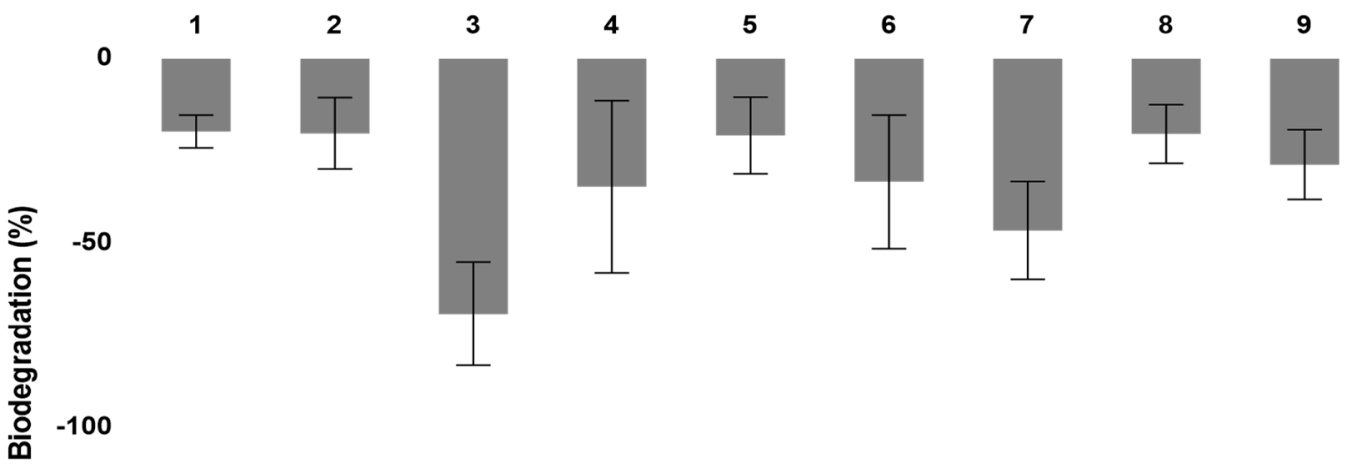

Control

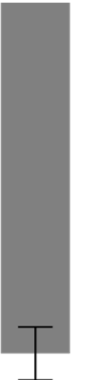

$-150$

One of the most potent (7-fold increase in modulus of elasticity) dentin biomodifiers described so far is an A-type trimer from pine bark extract. ${ }^{1}$ We have also tested two other A-type tetramers from cinnamon bark extracts that show promising dentin biomodification potential (data unpublished) 
S12 Purity analysis of compounds $\mathbf{1 - 9}$ using the $100 \%$ qHNMR method

qHNMR analysis of dimer $\mathbf{1}$ using the $100 \%$ method

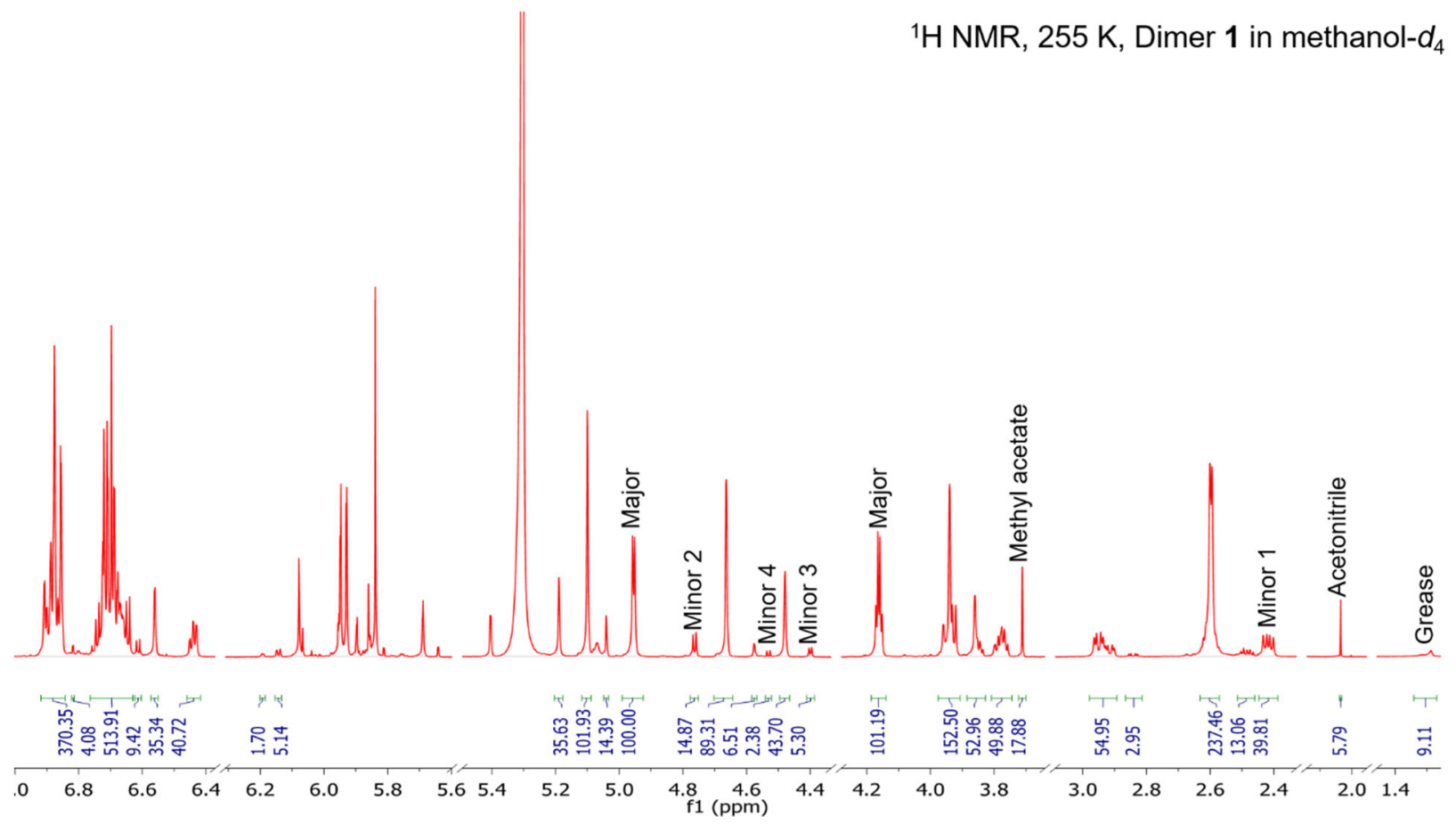

Cleanest integrals used for quantitation are labeled

\begin{tabular}{|c|c|c|c|c|c|c|c|c|c|}
\hline \multicolumn{10}{|c|}{$100 \%$ METHOD } \\
\hline \multirow[b]{2}{*}{$\begin{array}{l}\text { 혀 } \\
\text { 흔 }\end{array}$} & \multirow{2}{*}{\begin{tabular}{|l|} 
Dimer 1 (PA B1) \\
Major conformer
\end{tabular}} & \multicolumn{3}{|c|}{ (integration in MestReNova) } & \multirow[b]{2}{*}{$\begin{array}{c}\text { average } 1 \mathrm{H} \\
100.60\end{array}$} & \multirow[b]{2}{*}{$\begin{array}{c}\text { MW } \\
578.52\end{array}$} & \multirow[b]{2}{*}{$\begin{array}{c}\text { F (Molar Mass ratio) } \\
1.00\end{array}$} & \multirow[b]{2}{*}{$\begin{array}{c}\text { average mass } \\
100.60\end{array}$} & \multirow[b]{2}{*}{$\begin{array}{c}\delta \mathrm{H}[\mathrm{ppm}] \\
4.96(\mathrm{~d}) \\
4.16(\mathrm{dd}) \\
\end{array}$} \\
\hline & & $\begin{array}{c}\text { int } \\
100.00 \\
101.19\end{array}$ & $\begin{array}{c}\# \mathbf{H} \\
1 \\
1\end{array}$ & $\begin{array}{l}\text { int } 1 \mathrm{H} \\
100.00 \\
101.19\end{array}$ & & & & & \\
\hline & Minor 1 & 39.81 & \multirow{4}{*}{1} & 39.81 & 39.81 & \multirow{4}{*}{578.52} & \multirow{4}{*}{1.00} & 39.81 & $2.42(\mathrm{dd})$ \\
\hline & Minor 2 & 14.87 & & 14.87 & 14.87 & & & 14.87 & $4.76(d)$ \\
\hline & Minor 3 & 5.30 & & 5.30 & 5.30 & & & 5.30 & 4.40 (d) \\
\hline & Minor 4 & 2.38 & & 2.38 & 2.38 & & & 2.38 & $4.53(d)$ \\
\hline & Total minor & & & & 62.36 & & & 62.36 & \\
\hline \multicolumn{10}{|c|}{ Structurally unrelated impurities } \\
\hline & Acetonitrile & 5.79 & 3 & 1.93 & 1.93 & 41.05 & 0.07 & 0.14 & $2.03(\mathrm{~s})$ \\
\hline & Grease & 9.11 & 30 & 0.30 & 0.30 & 200.00 & 0.35 & 0.10 & $1.29(\mathrm{~s})$ \\
\hline & $\begin{array}{l}\text { unknown } \\
\text { (methyl acetate) }\end{array}$ & 17.88 & 3 & 5.96 & 5.96 & 74.08 & 0.13 & 0.76 & $3.71(s)$ \\
\hline & & & \multicolumn{2}{|c|}{ sum $1 \mathrm{H}$ of all } & 8.19 & & & 1.00 & \\
\hline & Mass ( $\%$ w/w) & & & & \multicolumn{2}{|c|}{ Molar (\% mol $/ \mathrm{mol})$} & & & \\
\hline \multicolumn{2}{|c|}{$\begin{array}{l}\text { 1H pure cmpd } \\
\text { (major confromer) }\end{array}$} & 100.60 & & & \multicolumn{2}{|c|}{$\begin{array}{l}\text { 1H pure cmpd } \\
\text { (major confromer) }\end{array}$} & 100.6 & & \\
\hline \multicolumn{2}{|c|}{$1 \mathrm{H}$ minor confromer } & 62.36 & & & \multicolumn{2}{|c|}{$1 \mathrm{H}$ minor confromer } & 62.36 & & \\
\hline \multicolumn{2}{|c|}{ sum $1 \mathrm{H}$ all imp } & 1.00 & & & \multicolumn{2}{|c|}{ sum $1 \mathrm{H}$ all imp } & 8.19 & & \\
\hline \multicolumn{2}{|c|}{$\%$ purity } & 99.01 & & & $\%$ purity & & 92.47 & & \\
\hline \multicolumn{2}{|c|}{$\%$ impurity } & 0.99 & & & $\%$ impurity & & 7.53 & & \\
\hline
\end{tabular}


qHNMR analysis of dimer 2 using the $100 \%$ method

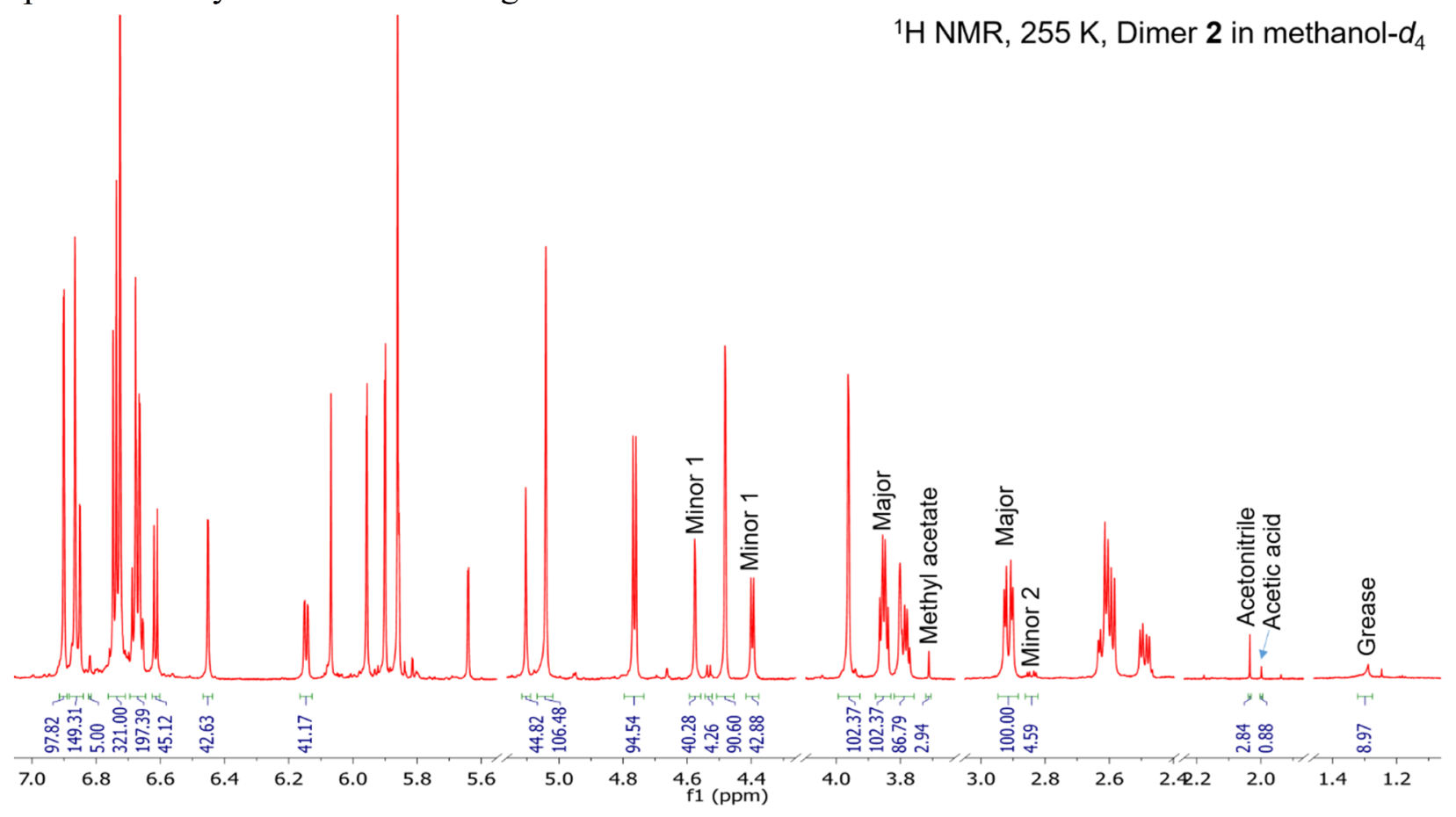

Cleanest integrals used for quantitation are labeled

\begin{tabular}{|c|c|c|c|c|c|c|c|c|c|}
\hline \multicolumn{10}{|c|}{$100 \%$ METHOD } \\
\hline \multirow[b]{2}{*}{ 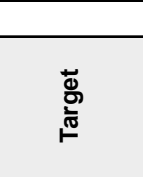 } & \multirow{2}{*}{\begin{tabular}{|l|} 
Dimer 2 \\
Major conformer
\end{tabular}} & \multicolumn{3}{|c|}{ (integration in MestReNova) } & & & & & \multirow[b]{2}{*}{$\begin{array}{c}\delta \mathrm{H} \text { [ppm] } \\
2.91(\mathrm{dd}) \\
3.85(\mathrm{dd})\end{array}$} \\
\hline & & $\begin{array}{c}\text { int } \\
100.00 \\
102.37\end{array}$ & $\begin{array}{c}\# \mathbf{H} \\
1 \\
1 \\
\end{array}$ & $\begin{array}{l}\text { int } 1 \mathbf{H} \\
100.00 \\
102.37\end{array}$ & $\begin{array}{c}\text { average } \mathbf{1 H} \\
101.19\end{array}$ & $\begin{array}{c}\text { MW } \\
578.52\end{array}$ & $\begin{array}{c}\boldsymbol{F} \text { (Molar Mass ratio) } \\
1.00\end{array}$ & $\begin{array}{c}\text { average mass } \\
101.19\end{array}$ & \\
\hline & Minor 1 & $\begin{array}{l}42.88 \\
40.28 \\
\end{array}$ & \multirow{2}{*}{1} & $\begin{array}{l}42.88 \\
40.28\end{array}$ & 41.58 & \multirow{2}{*}{578.52} & \multirow{2}{*}{1.00} & 41.58 & $\begin{array}{l}4.40(\mathrm{dd}) \\
4.57(\mathrm{brs})\end{array}$ \\
\hline & Minor 2 & $\begin{array}{l}4.59 \\
4.26\end{array}$ & & $\begin{array}{l}4.59 \\
4.26\end{array}$ & 4.43 & & & 4.43 & $\begin{array}{l}4.40(d) \\
4.53(d)\end{array}$ \\
\hline & Total minor & & & & 46.01 & & & 46.01 & \\
\hline \multicolumn{10}{|c|}{ Structurally unrelated impurities } \\
\hline & Acetonitrile & 2.84 & 3 & 0.95 & 0.95 & 41.05 & 0.07 & 0.07 & $2.03(\mathrm{~s})$ \\
\hline & Acetic acid & 0.88 & 3 & 0.29 & 0.29 & 60.05 & 0.10 & 0.03 & $2.00(\mathrm{~s})$ \\
\hline & Grease & 8.97 & 30 & 0.30 & 4.49 & 200.00 & 0.35 & 1.55 & $1.29(\mathrm{~s})$ \\
\hline & $\begin{array}{l}\text { unknown } \\
\text { (methyl acetate) }\end{array}$ & 2.94 & 3 & 0.98 & 0.98 & 74.08 & 0.13 & 0.13 & $3.71(\mathrm{~s})$ \\
\hline & & & \multicolumn{2}{|c|}{ sum of all $1 \mathrm{H}$ imp } & 6.71 & & & 1.78 & \\
\hline \multicolumn{2}{|c|}{ Mass ( $\%$ w/w) } & & & & \multicolumn{2}{|c|}{ Molar ( $\% \mathrm{~mol} / \mathrm{mol})$} & & & \\
\hline \multicolumn{2}{|c|}{$\begin{array}{l}1 \mathrm{H} \text { pure cmpd } \\
\text { (major confromer) }\end{array}$} & 101.19 & & & \multicolumn{2}{|c|}{$\begin{array}{l}1 \mathrm{H} \text { pure cmpd } \\
\text { (major confromer) }\end{array}$} & 101.19 & & \\
\hline \multicolumn{2}{|c|}{$1 \mathrm{H}$ minor confromer } & 46.01 & & & \multicolumn{2}{|c|}{$1 \mathrm{H}$ minor confromer } & 46.01 & & \\
\hline \multicolumn{2}{|c|}{ sum $1 \mathrm{H}$ all imp } & 1.78 & & & \multicolumn{2}{|c|}{ sum $1 \mathrm{H}$ all imp } & 6.71 & & \\
\hline$\%$ Major & & 67.92 & & & $\%$ Major & & 65.75 & & \\
\hline$\%$ Minor & & 30.88 & & & $\%$ Minor & & 29.89 & & \\
\hline$\%$ purity & & 98.81 & & & $\%$ purity & & 95.64 & & \\
\hline$\%$ Impurity & & 1.19 & & & $\%$ impurity & & 4.36 & & \\
\hline
\end{tabular}


qHNMR analysis of dimer 3 using the $100 \%$ method

${ }^{1} \mathrm{H}$ NMR, $255 \mathrm{~K}$, Dimer 3 in methanol- $d_{4}$

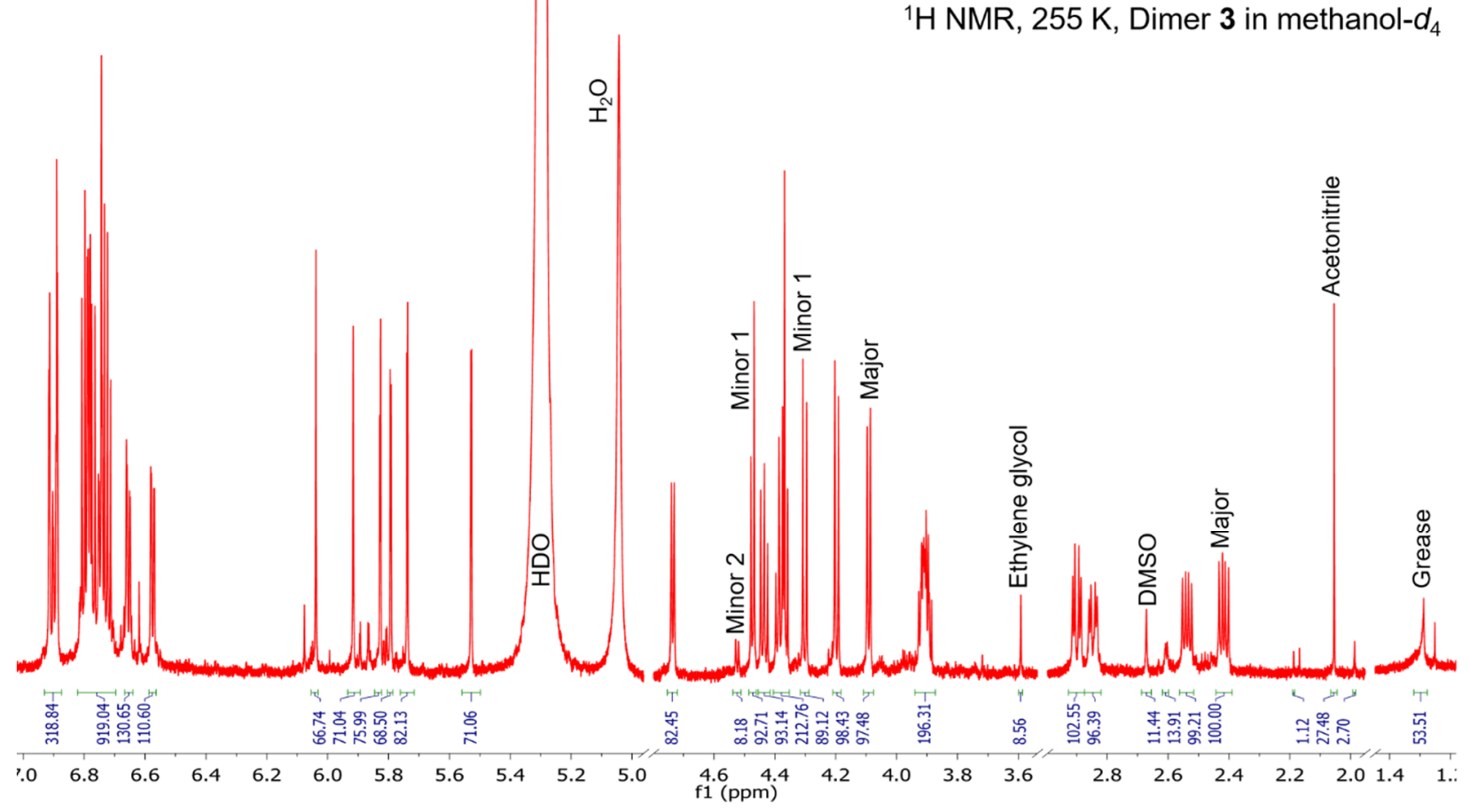

Cleanest integrals used for quantitation are labeled

\begin{tabular}{|c|c|c|c|c|c|c|c|c|c|}
\hline \multicolumn{10}{|c|}{$100 \%$ METHOD } \\
\hline \multirow[b]{2}{*}{ 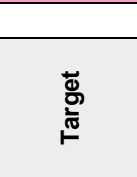 } & Dimer 3 & \multicolumn{3}{|c|}{ (integration in MestReNova) } & & & & & \\
\hline & Major conformer & $\begin{array}{c}\text { int } \\
100.00 \\
97.48 \\
\end{array}$ & $\begin{array}{c}\text { \# H } \\
1 \\
1 \\
\end{array}$ & $\begin{array}{c}\text { int } 1 \mathrm{H} \\
100.00 \\
97.48 \\
\end{array}$ & $\begin{array}{c}\text { average } \mathbf{1 H} \\
98.74\end{array}$ & $\begin{array}{c}\text { MW } \\
578.52\end{array}$ & $\begin{array}{c}\text { F (Molar Mass ratio) } \\
1.00\end{array}$ & $\begin{array}{c}\text { average mass } \\
98.74\end{array}$ & $\begin{array}{c}\delta \mathrm{H}[\mathrm{ppm}] \\
2.42(\mathrm{dd}) \\
4.09(\mathrm{~d})\end{array}$ \\
\hline & Minor 1 & $\begin{array}{l}89.12 \\
92.71\end{array}$ & \multirow{2}{*}{1} & $\begin{array}{l}89.12 \\
92.71\end{array}$ & 90.92 & \multirow{2}{*}{578.52} & \multirow{2}{*}{1.00} & 90.92 & $\begin{array}{c}4.30(\mathrm{dd}) \\
4.47(\mathrm{~d})\end{array}$ \\
\hline & Minor 2 & 8.18 & & 8.18 & 8.18 & & & 8.18 & $4.52(d)$ \\
\hline & Total minor & & & & 99.10 & & & 99.10 & \\
\hline \multicolumn{10}{|c|}{ Structurally unrelated impurities } \\
\hline & Acetonitrile & 27.48 & 3 & 9.16 & 9.16 & 41.05 & 0.07 & 0.65 & $2.03(\mathrm{~s})$ \\
\hline & ethylene glycol & 0.88 & 4 & 0.22 & 0.22 & 62.07 & 0.11 & 0.02 & $3.59(\mathrm{~s})$ \\
\hline & Grease & 8.97 & 30 & 0.30 & 0.30 & 200.00 & 0.35 & 0.10 & $1.29(\mathrm{~s})$ \\
\hline & DMSO & 11.44 & 6 & 1.91 & 1.91 & 78.13 & 0.14 & 0.26 & $2.65(s)$ \\
\hline & & & \multicolumn{2}{|c|}{ sum of all $1 \mathrm{H}$ imp } & 11.59 & & & 1.04 & \\
\hline \multicolumn{2}{|c|}{ Mass ( $\%$ w/w) } & & & & \multicolumn{2}{|c|}{ Molar (\% mol/mol) } & & & \\
\hline \multicolumn{2}{|c|}{$\begin{array}{l}\text { 1H pure cmpd } \\
\text { (major confromer) }\end{array}$} & 98.74 & & & \multicolumn{2}{|c|}{$\begin{array}{l}\text { 1H pure cmpd } \\
\text { (major confromer) }\end{array}$} & 98.74 & & \\
\hline \multicolumn{2}{|c|}{$1 \mathrm{H}$ minor confromer } & 99.10 & & & \multicolumn{2}{|c|}{ 1H minor confromer } & 99.10 & & \\
\hline \multicolumn{2}{|c|}{ sum $1 \mathrm{H}$ all imp } & 1.04 & & & \multicolumn{2}{|l|}{ sum $1 \mathrm{H}$ all imp } & 11.59 & & \\
\hline$\%$ Major & & 49.65 & & & $\%$ Major & & 47.15 & & \\
\hline$\%$ Minor & & 49.83 & & & $\%$ Minor & & 47.32 & & \\
\hline$\%$ purity & & 99.48 & & & $\%$ purity & & 94.47 & & \\
\hline$\%$ Impurity & & 0.52 & & & $\%$ impurity & & 5.53 & & \\
\hline
\end{tabular}


qHNMR analysis of dimer 4 using the $100 \%$ method

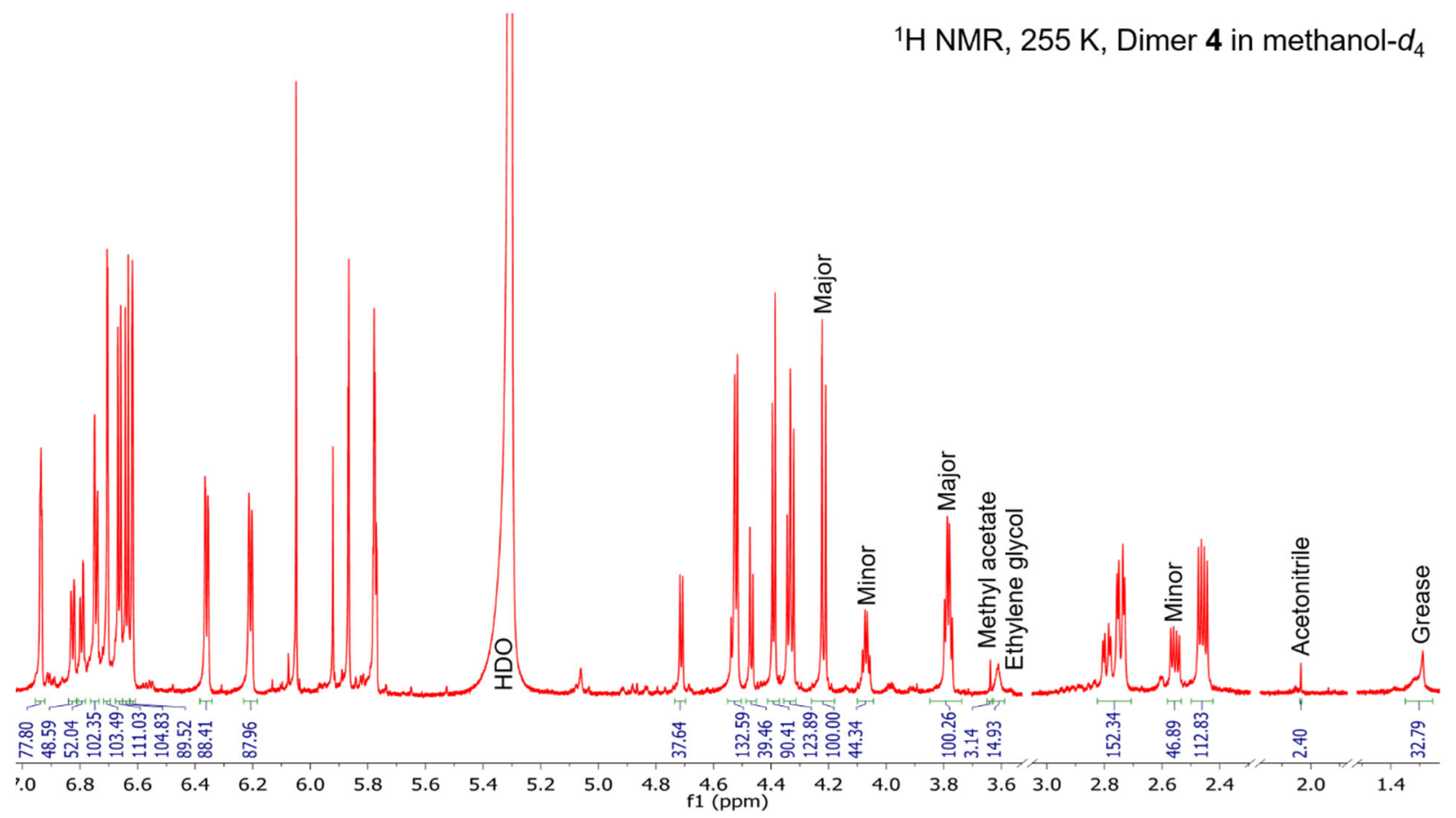

Cleanest integrals used for quantitation are labeled

$100 \%$ METHOD

\begin{tabular}{|c|c|c|c|c|c|c|c|c|c|}
\hline & \multirow{2}{*}{\multicolumn{3}{|c|}{ (integration in MestReNova) }} & & & \multirow[b]{3}{*}{$\begin{array}{c}\boldsymbol{F} \text { (Molar Mass ratio) } \\
1.00\end{array}$} & \multirow[b]{3}{*}{$\begin{array}{c}\text { average mass } \\
100.13\end{array}$} & \\
\hline \multicolumn{2}{|c|}{ Dimer 4 (procyanidin B3) } & & & & \multirow[b]{2}{*}{$\begin{array}{c}\text { average } \mathbf{1 H} \\
100.13\end{array}$} & \multirow[b]{2}{*}{$\begin{array}{c}\text { MW } \\
578.52\end{array}$} & & & \multirow[b]{2}{*}{$\begin{array}{c}\delta \mathrm{H}[\mathrm{ppm}] \\
4.22(\mathrm{~d}) \\
3.78(\mathrm{dd}) \\
\end{array}$} \\
\hline$\underset{\frac{\varpi}{\sigma}}{\frac{\varpi}{\sigma}}$ & Major conformer & $\begin{array}{c}\text { int } \\
100.00 \\
100.26 \\
\end{array}$ & $\begin{array}{c}\text { \# H } \\
1 \\
1 \\
\end{array}$ & $\begin{array}{l}\text { int } 1 \mathrm{H} \\
100.00 \\
100.26 \\
\end{array}$ & & & & & \\
\hline & \multirow[b]{2}{*}{ Minor conformer } & 46.89 & \multirow[b]{2}{*}{1} & 46.89 & \multirow{2}{*}{45.62} & \multirow{2}{*}{578.52} & \multirow[b]{2}{*}{1.00} & \multirow{2}{*}{45.62} & $2.56(\mathrm{dd})$ \\
\hline & & 44.34 & & 44.34 & & & & & $4.07(\mathrm{dd})$ \\
\hline & Total minor & & & & 45.62 & & & 45.62 & \\
\hline \multicolumn{10}{|c|}{ Structurally unrelated impurities } \\
\hline & Acetonitrile & 2.40 & 3 & 0.80 & \multirow{4}{*}{$\begin{array}{l}0.80 \\
3.73 \\
1.09 \\
3.81\end{array}$} & \multirow{4}{*}{$\begin{array}{c}41.05 \\
62.07 \\
200.00 \\
78.13\end{array}$} & \multirow{4}{*}{$\begin{array}{l}0.07 \\
0.11 \\
0.35 \\
0.14\end{array}$} & \multirow{4}{*}{$\begin{array}{l}0.06 \\
0.40 \\
0.38 \\
0.51\end{array}$} & $2.03(\mathrm{~s})$ \\
\hline & ethylene glycol & 14.93 & 4 & 3.73 & & & & & 3.59 (s) \\
\hline & Grease & 32.79 & 30 & 1.09 & & & & & $1.29(\mathrm{~s})$ \\
\hline & Methyl acetate & 11.44 & 3 & 3.81 & & & & & $3.64(s)$ \\
\hline & & & \multicolumn{2}{|c|}{ sum of all $1 \mathrm{H}$ imp } & 9.43 & & & 1.35 & \\
\hline \multicolumn{2}{|c|}{ Mass ( $\%$ w/w) } & & & & \multicolumn{2}{|c|}{ Molar (\% $\mathrm{mol} / \mathrm{mol})$} & & & \\
\hline \multicolumn{2}{|c|}{$\begin{array}{l}\text { 1H pure cmpd } \\
\text { (major confromer) }\end{array}$} & 100.13 & & & \multicolumn{2}{|c|}{$\begin{array}{l}\text { 1H pure cmpd } \\
\text { (major confromer) }\end{array}$} & 100.13 & & \\
\hline \multicolumn{2}{|c|}{$1 \mathrm{H}$ minor confromer } & 45.62 & & & \multicolumn{2}{|c|}{ 1H minor confromer } & \multicolumn{2}{|l|}{45.62} & \\
\hline \multicolumn{2}{|c|}{ sum $1 \mathrm{H}$ all imp } & 1.35 & & & \multicolumn{2}{|l|}{ sum $1 \mathrm{H}$ all imp } & 9.43 & & \\
\hline$\%$ Major & & 68.07 & & & $\%$ Major & & 64.53 & & \\
\hline$\%$ Minor & & 31.01 & & & $\%$ Minor & & 29.40 & & \\
\hline$\%$ purity & & 99.08 & & & $\%$ purity & & 93.92 & & \\
\hline$\%$ Impurity & & 0.92 & & & $\%$ impurity & & 6.08 & & \\
\hline
\end{tabular}


qHNMR analysis of dimer 5 using the $100 \%$ method

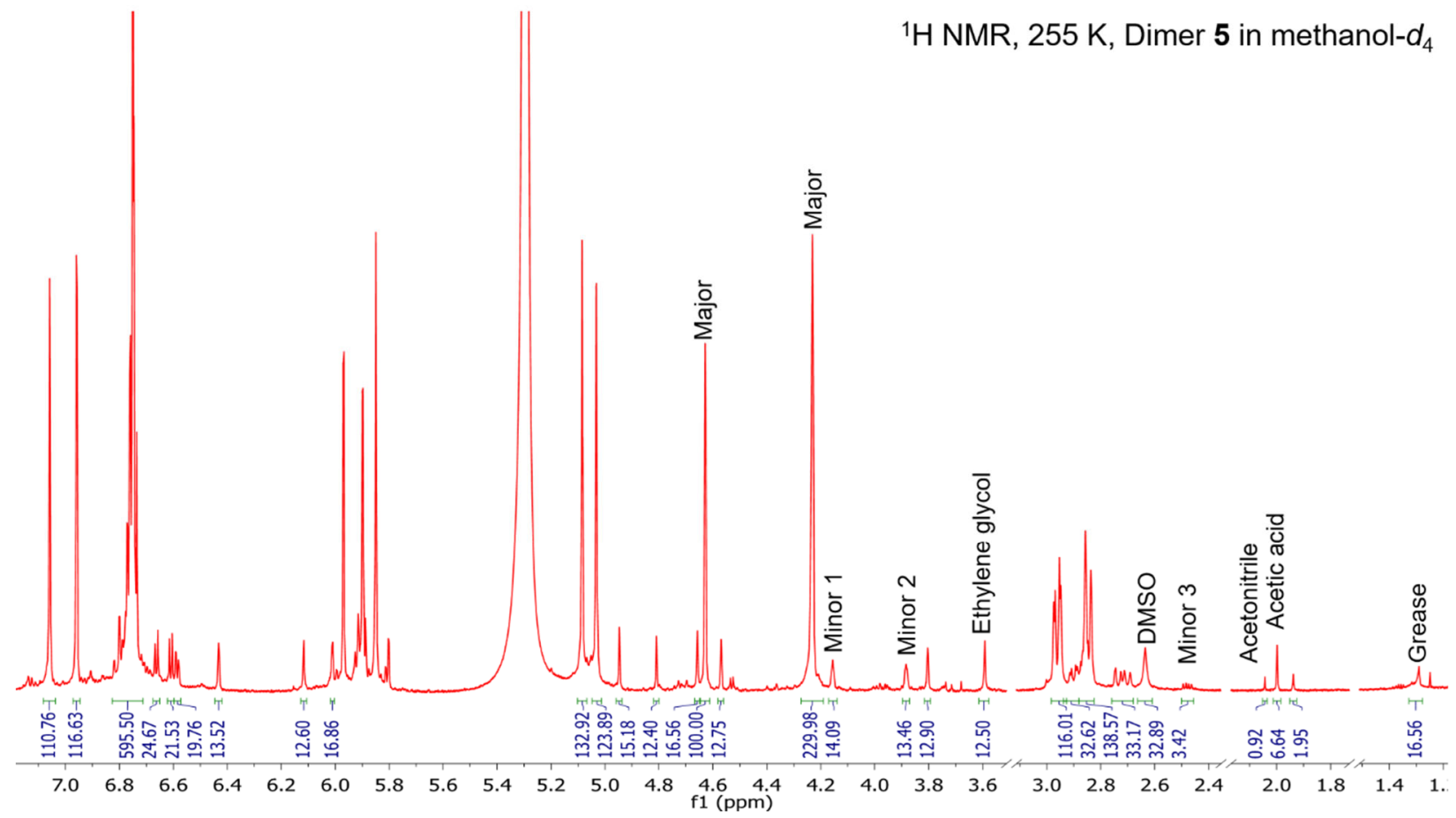

Cleanest integrals used for quantitation are labeled

\begin{tabular}{|c|c|c|c|c|c|c|c|c|c|}
\hline \multicolumn{10}{|c|}{$100 \%$ METHOD } \\
\hline \multirow[b]{2}{*}{$\frac{\overleftarrow{\Phi}}{\frac{\Phi}{\sigma}}$} & Dimer 5 & \multicolumn{3}{|c|}{ (integration in MestReNova) } & & & & & \multirow[b]{2}{*}{$\begin{array}{c}\delta \mathrm{H}[\mathrm{ppm} \\
4.63 \text { (brs) } \\
4.23\end{array}$} \\
\hline & Major conformer & $\begin{array}{c}\text { int } \\
100.00 \\
229.98\end{array}$ & $\begin{array}{c}\# \mathbf{H} \\
1 \\
2 \\
\end{array}$ & $\begin{array}{l}\text { int } 1 \mathbf{H} \\
100.00 \\
114.99\end{array}$ & $\begin{array}{c}\text { average } \mathbf{1 H} \\
107.50\end{array}$ & $\begin{array}{c}\text { MW } \\
578.52\end{array}$ & $\begin{array}{c}\boldsymbol{F} \text { (Molar Mass ratio) } \\
1.00\end{array}$ & $\begin{array}{c}\text { average mass } \\
107.50\end{array}$ & \\
\hline & $\begin{array}{l}\text { Minor } 1 \\
\text { Minor } 2 \\
\end{array}$ & $\begin{array}{l}14.09 \\
13.46 \\
\end{array}$ & \multirow{2}{*}{1} & $\begin{array}{l}14.09 \\
13.46 \\
\end{array}$ & $\begin{array}{l}14.09 \\
13.46\end{array}$ & \multirow{2}{*}{578.52} & & $\begin{array}{l}14.09 \\
13.46 \\
\end{array}$ & $\begin{array}{l}4.16(\mathrm{~m}) \\
3.89(\mathrm{~m})\end{array}$ \\
\hline & Minor 3 & 3.42 & & 3.42 & 3.42 & & & 3.42 & $2.48(d d)$ \\
\hline & Total minor & & & & 30.97 & & & 30.97 & \\
\hline \multicolumn{10}{|c|}{ Structurally unrelated impurities } \\
\hline & Acetonitrile & 0.92 & 3 & 0.31 & 0.31 & 41.05 & 0.07 & 0.02 & $2.03(\mathrm{~s})$ \\
\hline & ethylene glycol & 12.50 & 4 & 3.13 & 3.13 & 62.07 & 0.11 & 0.34 & $3.59(\mathrm{~s})$ \\
\hline & DMSO & 32.89 & 6 & 5.48 & 5.48 & 78.13 & 0.14 & 0.74 & $2.65(\mathrm{~s})$ \\
\hline & Grease & 16.56 & 30 & 0.55 & 0.55 & 200.00 & 0.35 & 0.19 & $1.29(\mathrm{~s})$ \\
\hline & Acetic acid & 11.44 & 3 & 3.81 & 3.81 & 60.05 & 0.10 & 0.40 & $3.64(s)$ \\
\hline & & & sum o & $1 \mathrm{H} \mathrm{imp}$ & 13.28 & & & 1.68 & \\
\hline \multicolumn{2}{|c|}{ Mass ( $\%$ w/w) } & & & & \multicolumn{2}{|c|}{ Molar (\% mol/mol) } & & & \\
\hline \multicolumn{2}{|c|}{$\begin{array}{l}1 \mathrm{H} \text { pure cmpd } \\
\text { (major confromer) }\end{array}$} & 107.50 & & & \multicolumn{2}{|c|}{$\begin{array}{l}1 \mathrm{H} \text { pure cmpd } \\
\text { (major confromer) }\end{array}$} & 107.5 & & \\
\hline \multicolumn{2}{|c|}{$1 \mathrm{H}$ minor confromer } & 30.97 & & & \multicolumn{2}{|c|}{$1 \mathrm{H}$ minor confromer } & 30.97 & & \\
\hline \multicolumn{2}{|c|}{ sum $1 \mathrm{H}$ all imp } & 1.68 & & & \multicolumn{2}{|c|}{ sum $1 \mathrm{H}$ all imp } & 13.28 & & \\
\hline$\%$ Major & & 76.70 & & & $\%$ Major & & 70.84 & & \\
\hline$\%$ Minor & & 22.10 & & & $\%$ Minor & & 20.41 & & \\
\hline$\%$ purity & & 98.80 & & & $\%$ purity & & 91.25 & & \\
\hline$\%$ Impurity & & 1.20 & & & $\%$ impurity & & 8.75 & & \\
\hline
\end{tabular}


qHNMR analysis of dimer 6 using the $100 \%$ method

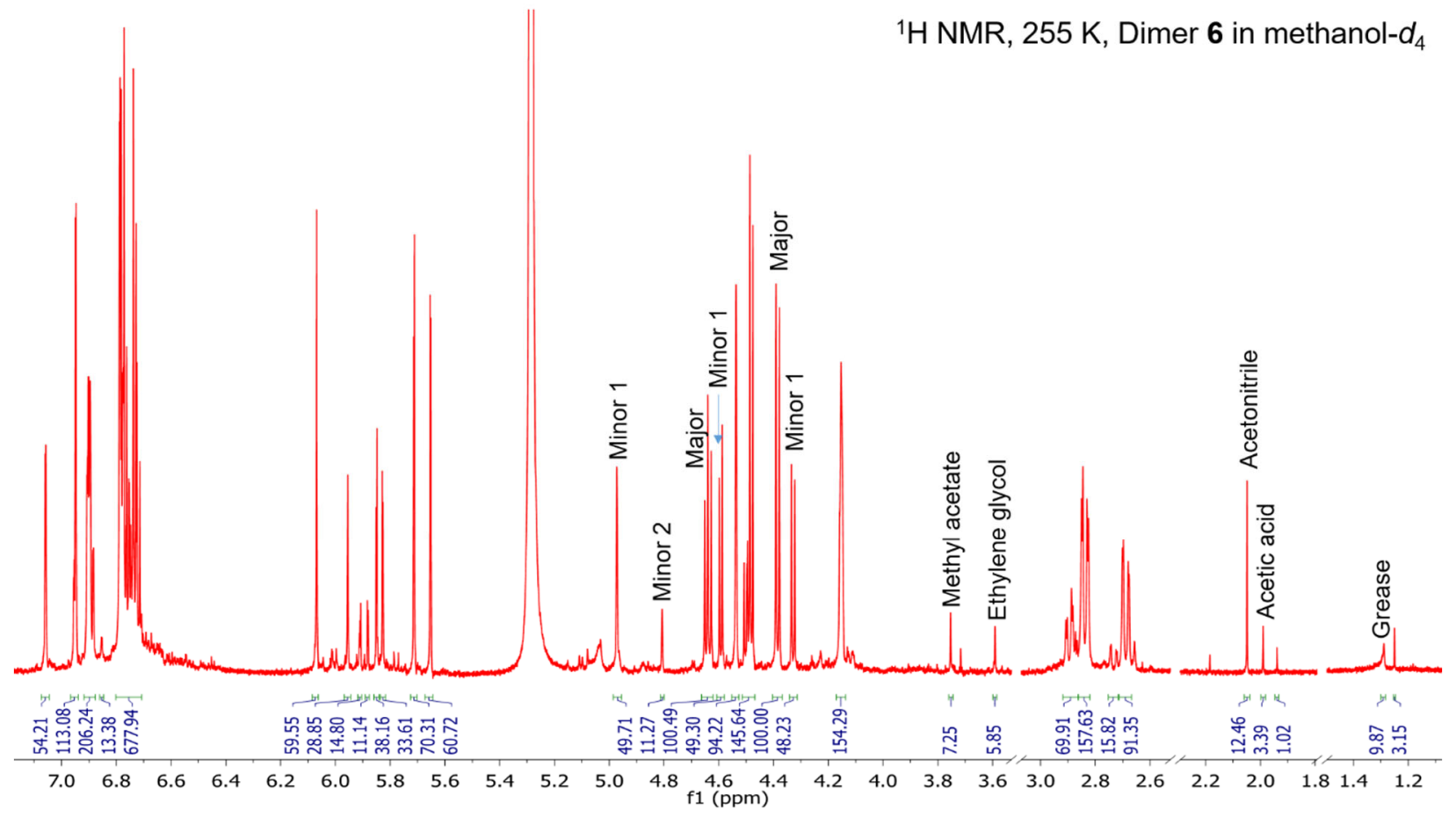

Cleanest integrals used for quantitation are labeled

\begin{tabular}{|c|c|c|c|c|c|c|c|c|c|}
\hline \multicolumn{10}{|c|}{$100 \%$ METHOD } \\
\hline \multirow[b]{2}{*}{ 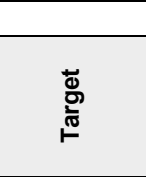 } & \multirow{2}{*}{\begin{tabular}{|l|} 
Dimer 6 \\
Major conformer
\end{tabular}} & \multicolumn{3}{|c|}{ (integration in MestReNova) } & & & & & \multirow[b]{2}{*}{$\begin{array}{c}\delta \mathrm{H}[\mathrm{ppm}] \\
4.38(\mathrm{~d}) \\
4.64(\mathrm{dd}) \\
\end{array}$} \\
\hline & & $\begin{array}{c}\text { int } \\
100.00 \\
100.49 \\
\end{array}$ & $\begin{array}{c}\# \mathbf{H} \\
1 \\
1 \\
\end{array}$ & $\begin{array}{l}\text { int } 1 \mathrm{H} \\
100.00 \\
100.49 \\
\end{array}$ & $\begin{array}{c}\text { average } \mathbf{1 H} \\
100.25\end{array}$ & $\begin{array}{c}\text { MW } \\
578.52\end{array}$ & $\begin{array}{c}\boldsymbol{F} \text { (Molar Mass ratio) } \\
1.00\end{array}$ & $\begin{array}{c}\text { average mass } \\
100.25\end{array}$ & \\
\hline & Minor 1 & $\begin{array}{l}48.23 \\
49.30 \\
\end{array}$ & \multirow{2}{*}{1} & $\begin{array}{l}48.23 \\
49.30 \\
\end{array}$ & 48.77 & \multirow{2}{*}{578.52} & \multirow{2}{*}{1.00} & 48.77 & $\begin{array}{l}4.33(\mathrm{~m}) \\
4.59(\mathrm{~m})\end{array}$ \\
\hline & Minor 2 & 11.27 & & 11.27 & 11.27 & & & 11.27 & $2.48(\mathrm{dd})$ \\
\hline & Total minor & & & & 60.04 & & & 60.04 & \\
\hline \multicolumn{10}{|c|}{ Structurally unrelated impurities } \\
\hline & Acetonitrile & 12.46 & 3 & 4.15 & \multirow{5}{*}{$\begin{array}{l}4.15 \\
1.46 \\
2.42 \\
0.33 \\
1.13\end{array}$} & \multirow{5}{*}{$\begin{array}{c}41.05 \\
62.07 \\
78.13 \\
200.00 \\
60.05\end{array}$} & \multirow{5}{*}{$\begin{array}{l}0.07 \\
0.11 \\
0.14 \\
0.35 \\
0.10\end{array}$} & \multirow{5}{*}{$\begin{array}{l}0.29 \\
0.16 \\
0.33 \\
0.11 \\
0.12\end{array}$} & $2.03(\mathrm{~s})$ \\
\hline & ethylene glycol & 5.85 & 4 & 1.46 & & & & & $3.59(\mathrm{~s})$ \\
\hline & Methyl acetate & 7.25 & 3 & 2.42 & & & & & $3.75(\mathrm{~s})$ \\
\hline & Grease & 9.87 & 30 & 0.33 & & & & & $1.29(\mathrm{~s})$ \\
\hline & Acetic acid & 3.39 & 3 & 1.13 & & & & & 1.99 (s) \\
\hline & & & \multicolumn{2}{|c|}{ sum of all $1 \mathrm{H}$ imp } & 9.49 & & & 1.01 & \\
\hline \multicolumn{2}{|c|}{ Mass ( $\%$ w/w) } & & & & \multicolumn{2}{|c|}{ Molar (\% mol/mol) } & & & \\
\hline \multicolumn{2}{|c|}{$\begin{array}{l}1 \mathrm{H} \text { pure cmpd } \\
\text { (major confromer) }\end{array}$} & 100.25 & & & \multicolumn{2}{|c|}{$\begin{array}{l}\text { 1H pure cmpd } \\
\text { (major confromer) }\end{array}$} & 100.25 & & \\
\hline \multicolumn{2}{|c|}{$1 \mathrm{H}$ minor confromer } & 60.04 & & & \multicolumn{2}{|c|}{ 1H minor confromer } & 60.04 & & \\
\hline \multicolumn{2}{|c|}{ sum $1 \mathrm{H}$ all imp } & 1.01 & & & \multicolumn{2}{|c|}{ sum $1 \mathrm{H}$ all imp } & 9.49 & & \\
\hline$\%$ Major & & 62.15 & & & $\%$ Major & & 59.05 & & \\
\hline$\%$ Minor & & 37.22 & & & $\%$ Minor & & 35.36 & & \\
\hline$\%$ purity & & 99.37 & & & $\%$ purity & & 94.41 & & \\
\hline$\%$ Impurity & & 0.63 & & & $\%$ impurity & & 5.59 & & \\
\hline
\end{tabular}


qHNMR analysis of dimer 7 using the $100 \%$ method

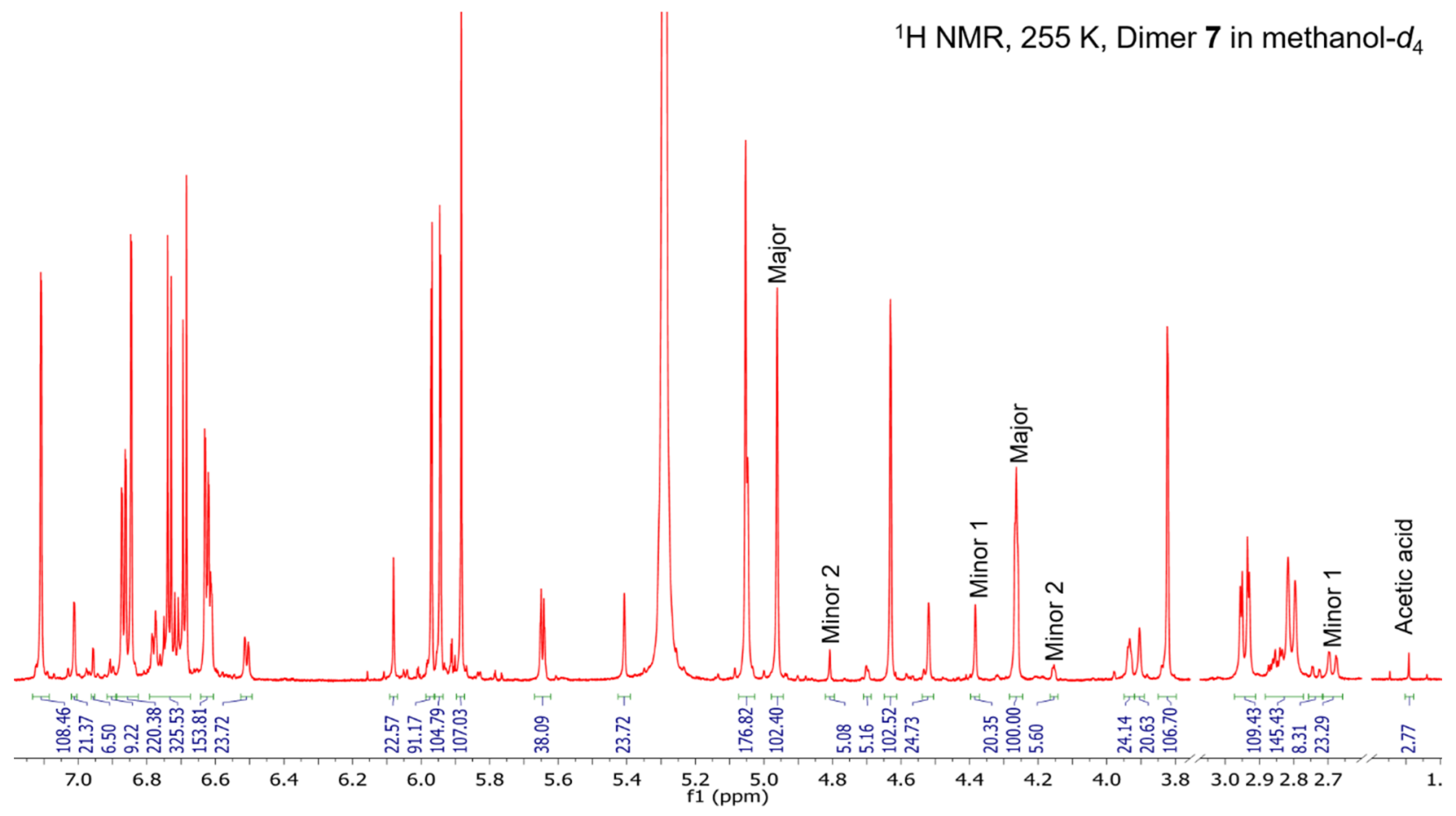

Cleanest integrals used for quantitation are labeled

\begin{tabular}{|c|c|c|c|c|c|c|c|c|c|}
\hline \multicolumn{10}{|c|}{ QUANTITATIVE ANALYSIS } \\
\hline \multicolumn{10}{|c|}{$100 \%$ METHOD } \\
\hline \multicolumn{2}{|c|}{ Dimer 7 (Procyanidin B2) } & \multicolumn{3}{|c|}{ (integration in MestReNova) } & & & & & \\
\hline 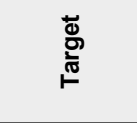 & Major conformer & $\begin{array}{c}\text { int } \\
100.00 \\
102.40 \\
\end{array}$ & $\begin{array}{c}\# \mathbf{H} \\
1 \\
1 \\
\end{array}$ & $\begin{array}{l}\text { int } 1 \mathrm{H} \\
100.00 \\
102.40 \\
\end{array}$ & $\begin{array}{c}\text { average } \mathbf{1 H} \\
101.20\end{array}$ & $\begin{array}{c}\text { MW } \\
578.52\end{array}$ & $\begin{array}{c}\text { F (Molar Mass ratio) } \\
1.00\end{array}$ & $\begin{array}{c}\text { average mass } \\
101.20\end{array}$ & $\begin{array}{c}\delta \mathrm{H} \text { [ppm] } \\
4.273 \text { (brs) } \\
4.96(\mathrm{~s}) \\
\end{array}$ \\
\hline & Minor 1 & $\begin{array}{l}23.29 \\
20.35 \\
\end{array}$ & \multirow{2}{*}{1} & $\begin{array}{l}23.29 \\
20.35 \\
\end{array}$ & 21.82 & \multirow{2}{*}{578.52} & \multirow{2}{*}{1.00} & 21.82 & $\begin{array}{l}2.69(\mathrm{dd}) \\
4.38(\mathrm{brs}) \\
\end{array}$ \\
\hline & Minor 2 & $\begin{array}{l}5.60 \\
5.08\end{array}$ & & $\begin{array}{l}5.60 \\
5.08\end{array}$ & 5.34 & & & 5.34 & $\begin{array}{l}4.16 \text { (brs) } \\
4.81 \text { (brs) }\end{array}$ \\
\hline & Total minor & & & & 27.16 & & & 27.16 & \\
\hline \multicolumn{10}{|c|}{ Structurally unrelated impurities } \\
\hline & Acetic acid & 2.77 & 3 & 0.92 & 0.92 & 60.05 & 0.10 & 0.10 & $1.99(\mathrm{~s})$ \\
\hline & & & \multicolumn{2}{|c|}{ sum of all $1 \mathrm{H}$ imp } & 0.92 & & & 0.10 & \\
\hline \multicolumn{2}{|c|}{ Mass ( $\%$ w/w) } & & & & \multicolumn{2}{|c|}{ Molar (\% mol/mol) } & & & \\
\hline \multicolumn{2}{|c|}{$\begin{array}{l}\text { 1H pure cmpd } \\
\text { (major confromer) }\end{array}$} & 101.20 & & & \multicolumn{2}{|c|}{$\begin{array}{l}\text { 1H pure cmpd } \\
\text { (major confromer) }\end{array}$} & 101.2 & & \\
\hline \multicolumn{2}{|c|}{$1 \mathrm{H}$ minor confromer } & 27.16 & & & \multicolumn{2}{|c|}{$1 \mathrm{H}$ minor confromer } & 27.16 & & \\
\hline \multicolumn{2}{|c|}{ sum $1 \mathrm{H}$ all imp } & 0.10 & & & \multicolumn{2}{|c|}{ sum $1 \mathrm{H}$ all imp } & 0.92 & & \\
\hline$\%$ Major & & 78.78 & & & $\%$ Major & & 78.28 & & \\
\hline$\%$ Minor & & 21.14 & & & $\%$ Minor & & 21.01 & & \\
\hline$\%$ purity & & 99.93 & & & $\%$ purity & & 99.29 & & \\
\hline$\%$ Impurity & & 0.07 & & & $\%$ impurity & & 0.71 & & \\
\hline
\end{tabular}


qHNMR analysis of trimer 8 using the $100 \%$ method

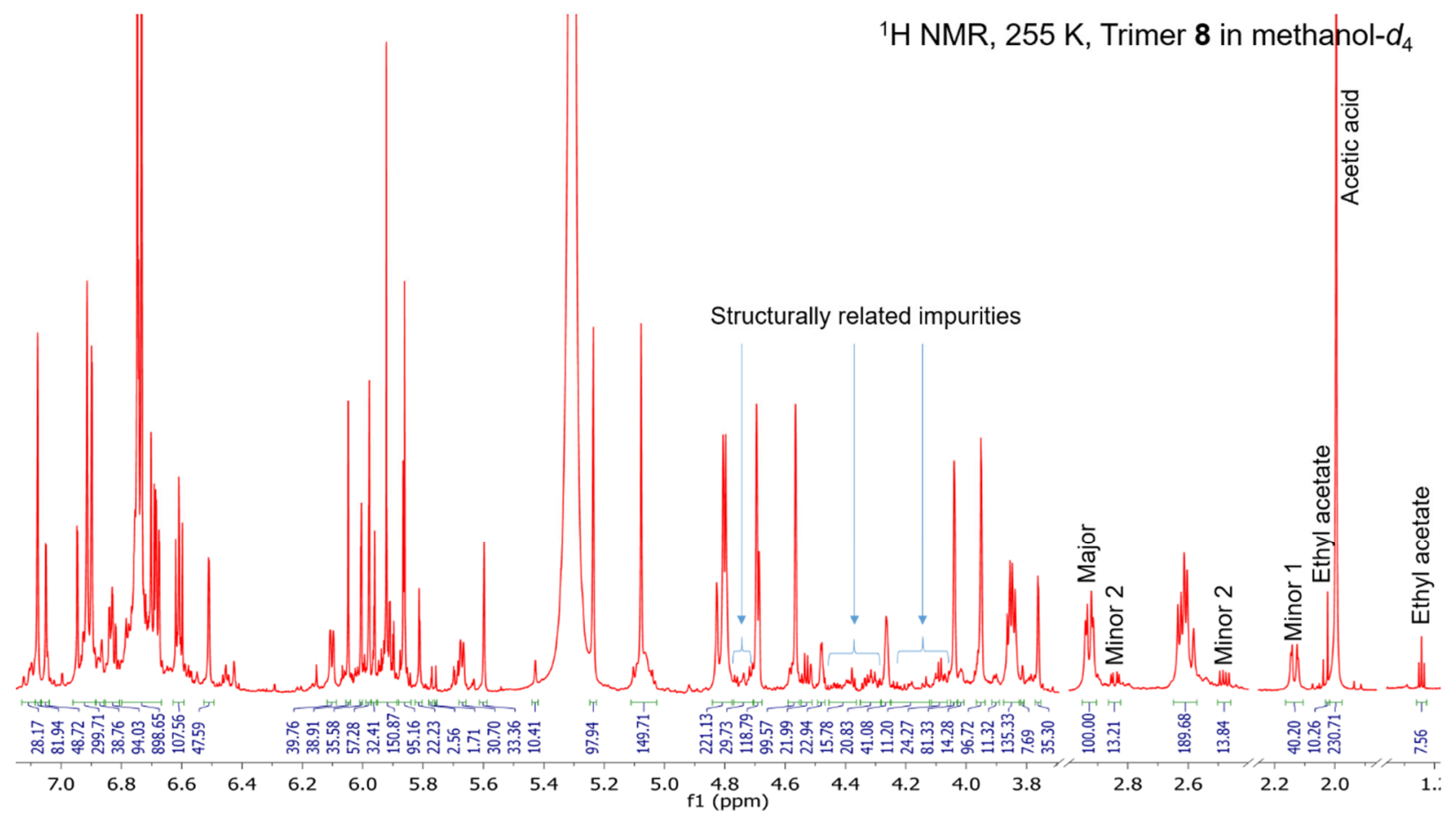

\begin{tabular}{|c|c|c|c|c|c|c|c|c|c|}
\hline \multirow[t]{4}{*}{ 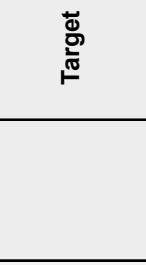 } & Major conformer & $\begin{array}{c}\text { int } \\
100.00 \\
100.72\end{array}$ & $\begin{array}{c}\# \mathbf{H} \\
1 \\
1\end{array}$ & $\begin{array}{l}\text { int } 1 \mathrm{H} \\
100.00 \\
100.72\end{array}$ & $\begin{array}{c}\text { average } 1 \mathbf{H} \\
100.36\end{array}$ & $\begin{array}{c}\text { MW } \\
578.52\end{array}$ & $\begin{array}{c}\text { F (Molar Mass ratio) } \\
1.00\end{array}$ & $\begin{array}{c}\text { average mass } \\
100.36\end{array}$ & $\begin{array}{l}\delta \mathrm{H}[\mathrm{ppm}] \\
3.99(\mathrm{brd}) \\
4.22(\mathrm{dd})\end{array}$ \\
\hline & Minor 2 & 8.21 & \multirow[b]{2}{*}{1} & 8.21 & 8.21 & \multirow[b]{2}{*}{578.52} & \multirow[b]{2}{*}{1.00} & 8.21 & $3.94(\mathrm{brs})$ \\
\hline & Minor 1 & $\begin{array}{l}14.69 \\
14.68\end{array}$ & & 14.69 & 14.69 & & & 14.69 & $\begin{array}{l}2.39(\mathrm{dd}) \\
3.86(\mathrm{brs})\end{array}$ \\
\hline & Total minor & & & & 22.90 & & & 22.90 & \\
\hline \multicolumn{10}{|c|}{ Structurally unrelated impurities } \\
\hline & \multirow{4}{*}{ n-butanol } & 161.56 & 3 & 53.85 & \multirow{4}{*}{52.28} & \multirow{4}{*}{74.12} & \multirow{4}{*}{0.13} & \multirow{4}{*}{6.70} & $0.95(t)$ \\
\hline & & 105.34 & 2 & 52.67 & & & & & $1.38(\mathrm{tq})$ \\
\hline & & 101.56 & 2 & 50.78 & & & & & $1.51(\mathrm{tt})$ \\
\hline & & 103.63 & 2 & 51.82 & & & & & $3.55(\mathrm{t})$ \\
\hline & \multirow{2}{*}{ Ethanol } & 10.96 & 3 & 3.65 & \multirow{2}{*}{2.90} & \multirow{2}{*}{46.06} & \multirow{2}{*}{0.08} & \multirow{2}{*}{0.23} & $1.18(\mathrm{t})$ \\
\hline & & 4.31 & 2 & 2.16 & & & & & $3.61(q)$ \\
\hline & Acetonitrile & 1.18 & 3 & 0.39 & 0.39 & 41.05 & 0.07 & 0.03 & $2.05(\mathrm{~s})$ \\
\hline & Acetic acid & 4.48 & 3 & 1.49 & 1.49 & 60.05 & 0.10 & 0.15 & $1.99(\mathrm{~s})$ \\
\hline & & & sum o & $1 \mathrm{H} \mathrm{imp}$ & 57.06 & & & 7.11 & \\
\hline \multicolumn{2}{|c|}{ Mass ( $\% w / w)$} & & & & \multicolumn{2}{|c|}{ Molar $(\% \mathrm{~mol} / \mathrm{mol})$} & & & \\
\hline \multicolumn{2}{|l|}{$1 \mathrm{H}$ pure cmpd } & 100.36 & & & \multicolumn{2}{|l|}{$1 \mathrm{H}$ pure $\mathrm{cmpd}$} & \multicolumn{2}{|l|}{100.36} & \\
\hline \multicolumn{2}{|c|}{$1 \mathrm{H}$ minor confromer } & 22.90 & & & \multicolumn{2}{|c|}{$1 \mathrm{H}$ minor confromer } & \multicolumn{2}{|l|}{22.90} & \\
\hline \multicolumn{2}{|c|}{ sum $1 \mathrm{H}$ all imp } & 7.11 & & & \multicolumn{2}{|l|}{ sum $1 \mathrm{H}$ all imp } & \multicolumn{2}{|l|}{57.06} & \\
\hline$\%$ Major & & 76.98 & & & $\%$ Major & & 55.66 & & \\
\hline$\%$ Minor & & 17.57 & & & $\%$ Minor & & 12.70 & & \\
\hline$\%$ purity & & 94.55 & & & $\%$ purity & & 68.35 & & \\
\hline$\%$ Impurity & & 5.45 & & & $\%$ impurity & & 31.65 & & \\
\hline
\end{tabular}


qHNMR analysis of trimer 9 using the $100 \%$ method

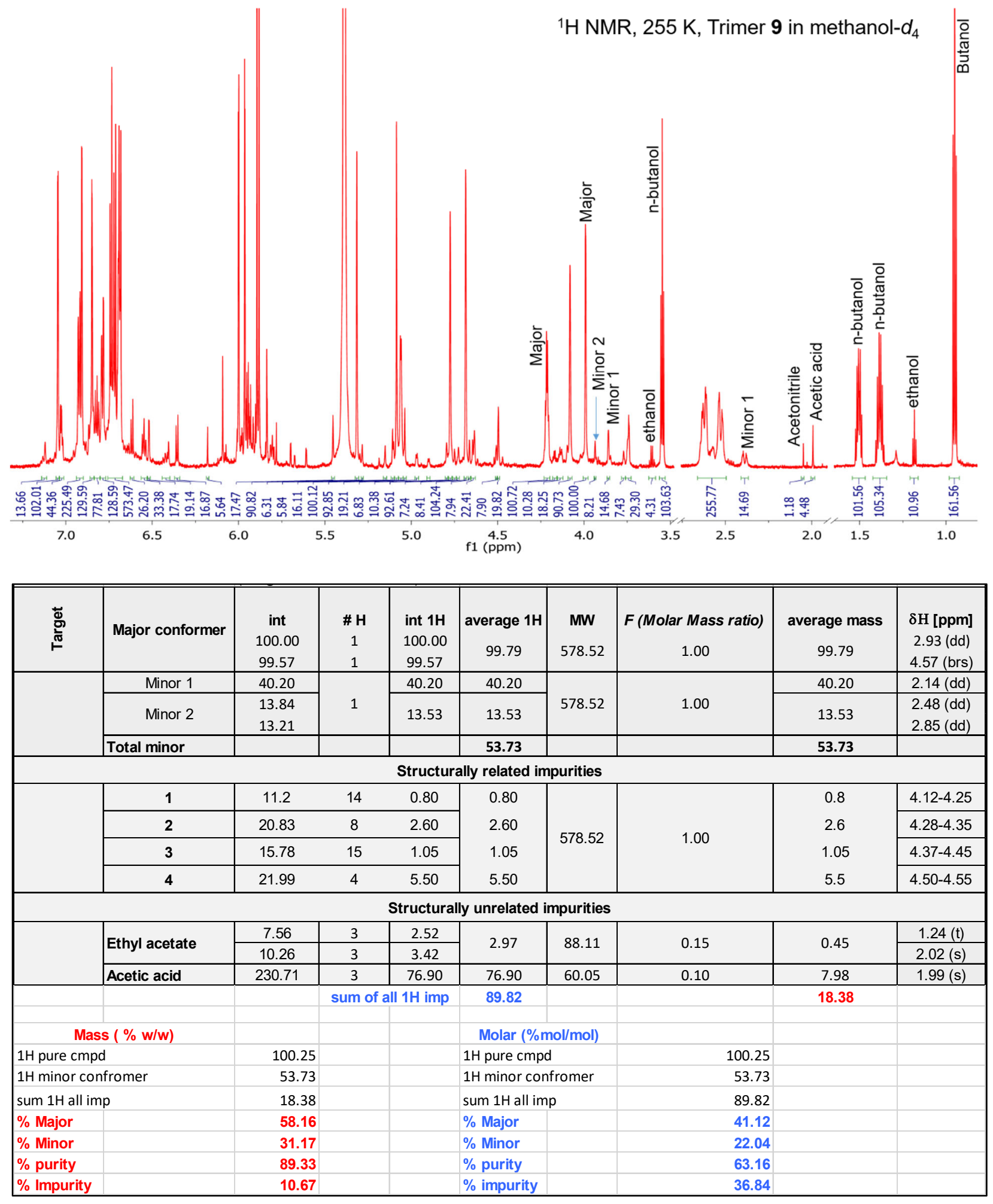




\section{References:}

(1) Nam, J.-W. W.; Phansalkar, R. S.; Lankin, D. C. D. C.; Bisson, J.; McAlpine, J. B. J. B.; Leme, A. A. A.; Vidal, C. M. P. C. M. P.; Ramirez, B.; Niemitz, M.; Bedran-Russo, A.; Chen, S.-N. S.-N.; Pauli, G. F. G. F. J. Org. Chem. 2015, 80 (15), 7495-7507. 